\title{
ON THE USE OF SHOT NOISE FOR PHOTON COUNTING
}

\author{
JONAS ZMUIDZINAS \\ Division of Physics, Mathematics, and Astronomy, California Institute Institute of Technology, Pasadena, CA 91125, USA; jonas@caltech.edu \\ Received 2015 January 13; accepted 2015 September 8; published 2015 October 22
}

\begin{abstract}
Lieu et al. have recently claimed that it is possible to substantially improve the sensitivity of radio-astronomical observations. In essence, their proposal is to make use of the intensity of the photon shot noise as a measure of the photon arrival rate. Lieu et al. provide a detailed quantum-mechanical calculation of a proposed measurement scheme that uses two detectors and conclude that this scheme avoids the sensitivity degradation that is associated with photon bunching. If correct, this result could have a profound impact on radio astronomy. Here I present a detailed analysis of the sensitivity attainable using shot-noise measurement schemes that use either one or two detectors, and demonstrate that neither scheme can avoid the photon bunching penalty. I perform both semiclassical and fully quantum calculations of the sensitivity, obtaining consistent results, and provide a formal proof of the equivalence of these two approaches. These direct calculations are furthermore shown to be consistent with an indirect argument based on a correlation method that establishes an independent limit to the sensitivity of shot-noise measurement schemes. Furthermore, these calculations are directly applicable to the regime of interest identified by Lieu et al. Collectively, these results conclusively demonstrate that the photon-bunching sensitivity penalty applies to shot-noise measurement schemes just as it does to ordinary photon counting, in contradiction to the fundamental claim made by Lieu et al. The source of this contradiction is traced to a logical fallacy in their argument.
\end{abstract}

Key words: instrumentation: miscellaneous - techniques: miscellaneous

\section{INTRODUCTION}

In the infrared, optical, or X-ray bands, detection sensitivities are ultimately limited by the Poisson statistics of photon counting, with rms count fluctuations given by $\sqrt{N}$ where $N$ is the mean number of photons collected (Gehrels 1986). Thus the Poisson uncertainty in the flux measured for an astronomical source is proportional to the square root of the total intensity of the radiation falling on the detector. Meanwhile, sensitivities for radio-astronomical observations are calculated using the radiometer equation (Dicke 1946), which states that the measurement uncertainty is proportional to the the total radiation intensity rather than its square root.

A transition between these two regimes - radio and opticalis therefore inevitable, and corresponds to a shift from a classical description involving fields and waves to a quantum description involving photons, sometimes referred to as the radio-optical dichotomy (Nityananda 1994) and ultimately stemming from the wave-particle duality of quantum mechanics. The nature of this transition was clarified through the demonstration of correlated photon arrivals at two independent detectors by Hanbury Brown \& Twiss (1956; HBT), using a setup similar to that illustrated in Figure 1. The HBT correlations are a manifestation of photon bunching, which causes the photon arrivals to be clustered in time rather than being purely random. As described in more detail in Section 2, bunching causes the photon count fluctuations for a single detector to increase to $\sqrt{N(1+n)}$ rather than the usual $\sqrt{N}$ for Poisson statistics. Here $n$ represents the photon mode occupation number for a detector with unit efficiency (Zmuidzinas 2003), given by the Bose-

\footnotetext{
1 Thermal radiation at optical wavelengths with high occupation number $n$ may readily be generated in the laboratory using stochastically modulated coherent laser radiation, e.g., produced by scattering from a rotating ground glass plate (Rousseau 1971).
}

Einstein formula $n=1 /\left(e^{h \nu / k T}-1\right)$ for thermal blackbody radiation at a temperature $T$. Bunching is usually ignorable for astronomical observations made in the infrared to X-ray bands because $n \ll 11^{1}$ in contrast, photon bunching is a large effect in the radio band since $n \sim k T / h \nu \gg 1$. Furthermore, at radio wavelengths both $N$ and $n$ scale with the intensity of the radiation being detected; therefore, $\sqrt{N(1+n)}$ is also proportional to the intensity, in agreement with the Dicke equation.

In a recent paper, Lieu et al. (2015) claim to have found a method for avoiding the extra noise associated with photon bunching and thereby potentially increasing the sensitivity of radio telescopes by $\sqrt{1+n}$, which is a large factor, e.g., over an order of magnitude for the example of Arecibo described in their paper. Such a possibility is of obvious interest given the large sums spent on the construction of radio telescopes and the associated receiving equipment. The essence of the Lieu et al. (2015) proposal is to use the wide-band shot noise at the output of a fast photon-counting detector as a measure of the radiation intensity. ${ }^{2}$ Consider the simple single-detector setup illustrated in Figure 2. It is helpful to visualize the noise spectrum (the power spectral density, or PSD) at the output of the detector as illustrated in Figure 3, which graphically summarizes the quantum-mechanical calculations presented later in Section 2. The shot-noise spectrum (Schottky 1918) is white and featureless within the output bandwidth of the detector, and has an intensity that is proportional to the mean photon arrival rate $\bar{\Gamma}$. Meanwhile, the bunching noise component is confined to lower frequencies, determined by the bandwidth $\Delta \nu$ of the radiation being detected. In principle, the radiation bandwidth $\Delta \nu$ may be made arbitrarily small using narrow-band filters

\footnotetext{
2 A fast photon detector operating at radio frequencies may represent a serious technical challenge, but not one of fundamental principle: tunnel junction detectors offer one possible method of implementation (Tucker \& Millea 1978; Schoelkopf et al. 1999).
} 


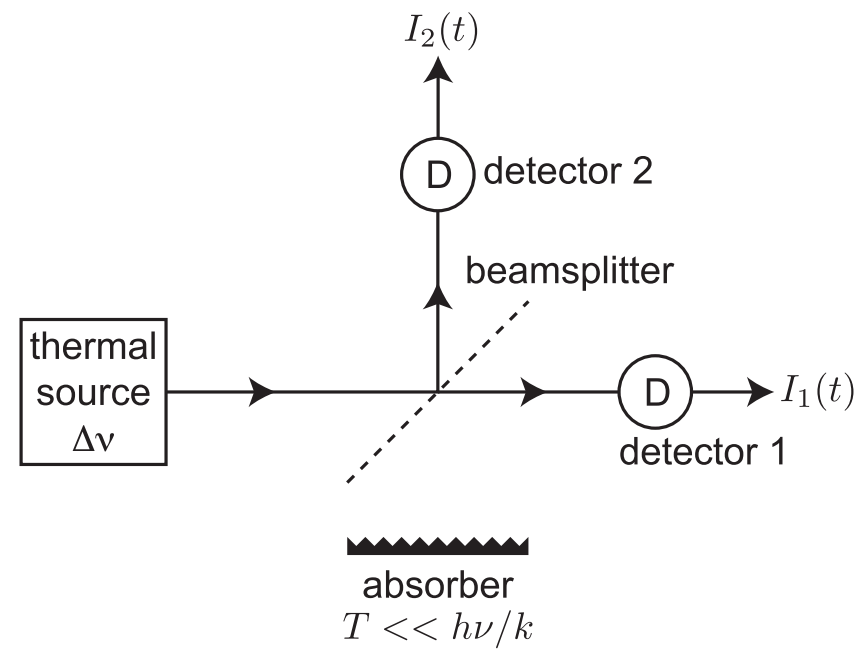

Figure 1. Two-detector experiment similar to that used by Hanbury Brown \& Twiss (1956) and others (e.g., Harwit 1960) to demonstrate photon correlations. A bright thermal light source with narrow spectral bandwidth $\Delta \nu$ illuminates two photon detectors via a $50 / 50$ beamsplitter, producing photocurrents $I_{1}(t)$ and $I_{2}(t)$. Photon bunching results in a nonzero correlation between the photocurrents: $\delta I_{1}(t) \delta I_{2}(t) \neq 0$. The unused input port of the beamsplitter is terminated with a cold (dark) absorber to prevent stray light from entering.

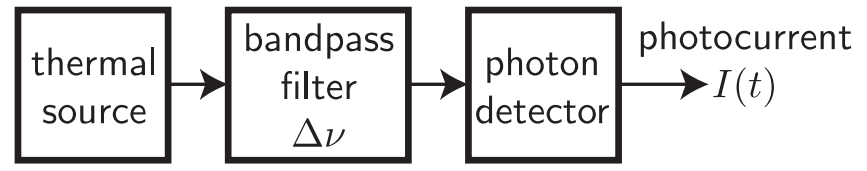

Figure 2. Simple setup consisting of a thermal radiation source, an optical bandpass filter with transmission bandwidth $\Delta \nu$, and an ideal photon detector. The detector output is represented by the photocurrent $I(t)$. The thermal source and filter comprise the light source in Figure 1.

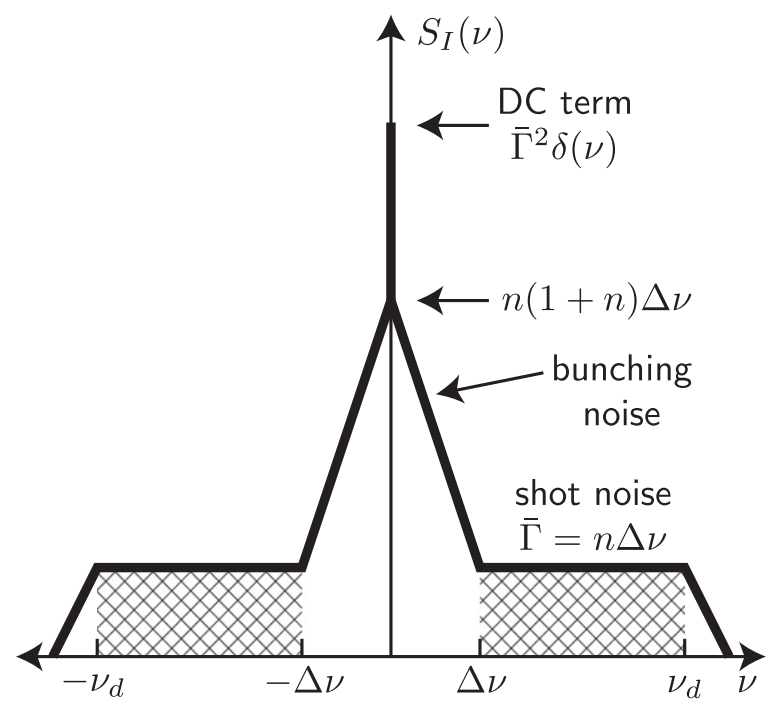

Figure 3. Photocurrent noise spectrum $S_{I}(\nu)$ for an ideal photon detector illuminated with filtered thermal radiation consists of three components: (1) a DC term contributed by the mean photocurrent; (2) a photon bunching component that extends to $\pm \Delta \nu$, where $\Delta \nu$ is the bandwidth of the radiation; (3) a white shot noise term that rolls off at $\pm \nu_{\mathrm{d}}$, the detector bandwidth. Both the DC photocurrent and the shot noise intensity are proportional to the average photon arrival rate $\bar{\Gamma}=n \Delta \nu$. The shaded region shows the portion of the spectrum that is available for measurement of the shot noise intensity without interference from the bunching noise component. See Section 2 and Equation (20) for details.

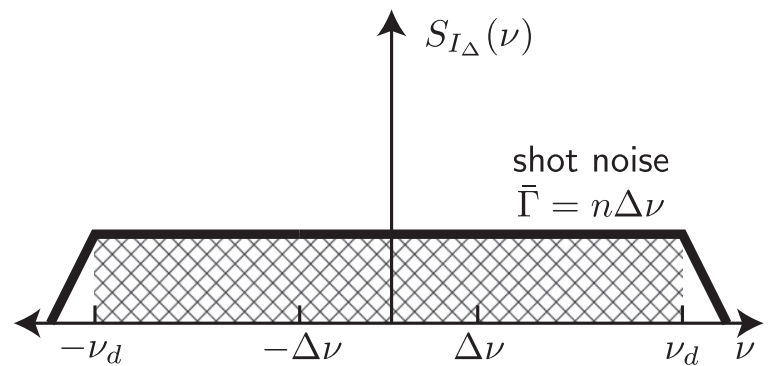

Figure 4. Noise spectrum $S_{I_{\Delta}}(\nu)$ for the output $I_{\Delta}$ of the two-detector scheme proposed by Lieu et al. (2015) and illustrated in Figure 1. Here $I_{\Delta}=I_{1}-I_{2}$ represents the difference in the photocurrents for the two detectors. Taking this difference eliminates the DC component as well as the bunching component in the spectrum, leaving only the white shot noise component that is proportional to the mean photon rate $\bar{\Gamma}$. The full spectrum is available for measurement of the shot noise intensity, as illustrated by the shaded region. See Section 3 and Equations (39)-(41) for details.

preceding the detector, so the use of a fast detector with an output bandwidth $\nu_{\mathrm{d}} \gg \Delta \nu$ allows the region of the photocurrent noise spectrum where the white shot noise dominates to be accessed and measured with appropriate signal processing techniques. Clearly, it is advantageous to use a large measurement bandwidth $B=\nu_{\mathrm{d}}-\Delta \nu$, since the fractional precision with which the shot noise intensity may be determined cannot be better than $1 / \sqrt{B T}$, where $T$ is the measurement time (Dicke 1946).

Alternatively, as specifically proposed by Lieu et al. (2015) and shown in Figure 1, a 50/50 beamsplitter or its radio equivalent may be used to feed two detectors. Each detector individually has an output noise spectrum similar to that shown in Figure 3, although with half the total photon rate $(\bar{\Gamma} / 2)$ per detector. The DC term may be eliminated by taking the difference of the two photocurrents. Differencing also eliminates the bunching noise lying in the frequency interval $[-\Delta \nu, \Delta \nu]$, because this component is fully correlated at the two detectors, as is demonstrated through a quantum-mechanical calculation in Section 3. Indeed, this component is responsible for the HBT correlations. Thus, only the white shot-noise spectrum survives after taking the difference, as shown in Figure 4; the shot noise intensity may then be measured using relatively simple signal processing techniques. Although the measurement bandwidth may now be increased to $B=\nu_{\mathrm{d}}$ instead of $\nu_{\mathrm{d}}-\Delta \nu$, the resulting improvement is modest when $\nu_{\mathrm{d}} \gg \Delta \nu$. Lieu et al. (2015) present a full quantum-mechanical calculation of the sensitivity of such a shot noise measurement scheme using two detectors, which is a nontrivial task involving computation of eighth-order moments of photon operators, and conclude that the $\sqrt{N}$ Poisson uncertainty may be achieved instead of the usual bunchingdegraded $\sqrt{N(1+n)}$ uncertainty as expressed by the Dicke equation. This result is quite surprising, and if correct and amenable to practical implementation, would represent a significant discovery with the potential to stimulate large advances in radio astronomy. However, as demonstrated in Section 10, the fundamental conclusion of the Lieu et al. (2015) paper rests on a logical fallacy and is therefore not valid. Section 10 also contains a simple intuitive argument that demonstrates why the measurement scheme proposed by Lieu et al. is in fact subject to the photon bunching penalty; those uninterested in the detailed calculations in the following sections may wish to jump straight to Section 10. 


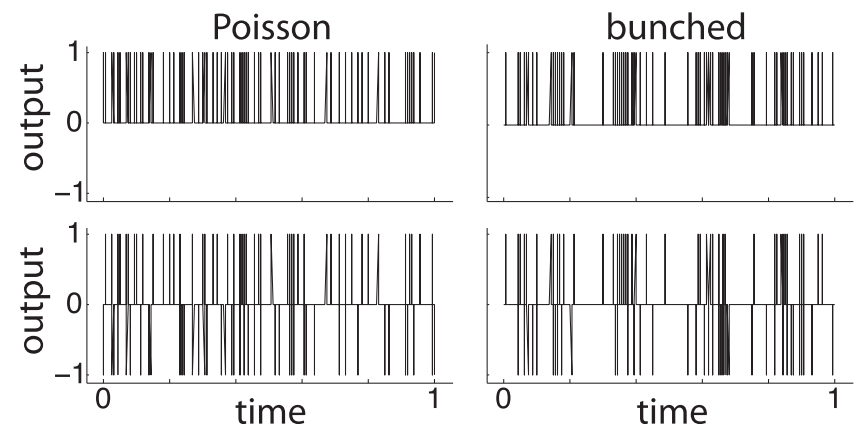

Figure 5. Top left: simulated output time stream for a single detector (see diagram in Figure 2) illuminated by a coherent source or a thermal source with low occupation number. The photon arrival rate is constant with time, which is the case of Poisson statistics. Bottom left: differenced output of a beamsplitterfed pair of detectors (Figure 1) for the same Poisson case. Top right: output time stream for a single detector illuminated with strongly bunched thermal radiation (high occupation number). Bottom right: differenced output of a detector pair for the bunched case. Horizontal and vertical scales are in arbitrary units.

The work of Lieu et al. (2015), and the quantum calculations presented here, may pose a challenge to those who are more familiar with classical concepts such as fields and voltages than with photon operators and quantum mechanics. Nonetheless, the essence of the problem is quite easy to understand by use of a familiar analogy. The analogy relies on the fact that thermal photon bunching can be correctly described by a photon arrival rate that varies with time in a random way, as will be discussed below. Imagine listening to the sound of rain landing on a roof: this is the shot noise produced by the random arrivals of a large number of individual raindrops. The intensity of the sound depends on how hard it is raining, i.e., the arrival rate of the raindrops. ${ }^{3}$ If the raindrop arrival rate changes with time, as often occurs over timescales of seconds to minutes, the intensity of the sound will vary accordingly. Thus, while a measurement of the total precipitation may be made by integrating the acoustic shot noise intensity over time, this shot noise measurement will reflect the fluctuations of the raindrop arrival rate in the same way as would a direct measurement, e.g., observations of the water level in a standard rain gauge. Note that the spectral character or the "sound" of the acoustic rain noise remains constant as the intensity changes. Furthermore, note that the connection between the two measurement methods - acoustic noise versus rain gauge -is purely classical and has nothing to do with quantum mechanics. This statement is also true for the photon detection problem. Indeed, the output of a photodetector is an entirely classical quantity - a train of electrical pulses - whose properties are fully specified by the statistics of the photon arrival times. While we may need to turn to quantum mechanics to calculate the arrival time statistics, once the arrival time statistics are known, in principle we can generate a simulated classical pulse train numerically, as illustrated in Figure 5, and use this time stream to calculate any other quantity of interest, e.g., the mean and variance of the photon counts, or the mean and variance of the shot noise intensity, or the correlation between the photon counts and shot noise intensity, etc. These quantities are all related to various moments of the same classical time stream. It is therefore not surprising that bunching affects standard photon counting measurements and

\footnotetext{
The raindrop size should be kept fixed for the analogy to hold.
}

photon shot noise measurements in similar ways, and therefore the sensitivity degradation due to bunching cannot be avoided. Indeed, in Section 8 I present a calculation that demonstrates that the shot noise intensity has a nonzero correlation with the photon counts, and then use this correlation to establish a rigorous sensitivity bound for the shot noise measurement. This bound shows that the shot noise measurement is subject to the same $\sqrt{1+n}$ sensitivity degradation due to photon bunching as for ordinary photon counting.

Let us continue to accept the claim that photon bunching can be correctly described by a photon arrival rate that varies randomly with time. Would we expect to see the noise spectra illustrated in Figures 3 and 4, which were derived from the quantum calculations presented in Sections 2 and 3? It is helpful to visualize the detector outputs as a function of time as shown in Figure 5. The detector bandwidth is assumed to be larger than the photon arrival rate, $\nu_{\mathrm{d}}>\bar{\Gamma}$, so the detected photons are visible as sharp, well-separated output pulses. For the case of two detectors, taking the difference of the two outputs means that the pulses may be positive or negative depending on which detector receives the photon. The two subplots on the left correspond to the case that the photon arrival rate is kept constant, which produces Poisson statistics; in contrast, the two subplots on the right were generated using a time-variable photon arrival rate in order to simulate photon bunching. Imagine that these output time streams are averaged over a timescale $\tau$ that is long compared to $1 / \bar{\Gamma}$. For the single detector case, Poisson arrivals would produce a DC component along with small fractional fluctuations of order $1 / \sqrt{\bar{\Gamma} \tau}$. Meanwhile, a time-variable arrival rate would lead to a DC component along with significantly larger fluctuations, in accordance with the noise spectrum shown in Figure 3. For two detectors, the positive and negative pulses largely cancel when performing the time average. This cancellation occurs for both the Poisson and bunched cases, in agreement with the noise spectrum shown in Figure 4. Furthermore, both positive and negative pulses deliver the same high-frequency energy, on average, to the subsequent circuitry and thus contribute equally to the shot noise intensity. Therefore, the shot noise intensity for the single-detector and two-detector cases should be the same. Thus, our conclusion is that a description of photon bunching in which the photon arrival rate varies randomly with time could indeed reproduce the noise spectra in Figures 3 and 4.

Is it in fact correct to view photon bunching as resulting from a time-varying photon arrival rate? Is Figure 5 a faithful depiction of photon bunching? Indeed, this was how Hanbury Brown \& Twiss (1957) viewed the phenomenon in their original work. In their words: “... we are dealing essentially with an interference phenomenon which can be interpreted, on the classical wave picture, as a correlation between intensity fluctuations due to beats between waves of different frequency; the concept of a photon need only be introduced at the stage where energy is extracted from the light beam in the process of photoemission." This physical picture is why the excess noise due to photon bunching continues to be referred to as "wave noise." Hanbury Brown \& Twiss (1957) computed the effect using exactly this semiclassical approach, in which the light is first treated as a classical wave, consisting of a random superposition of components at different frequencies, resulting in an intensity that has fractional variations of order unity that occur on a "coherence" timescale $\tau \sim \Delta \nu^{-1}$ that is set by the 
fastest beat frequency that can be produced if the spectrum is restricted to an optical bandwidth $\Delta \nu$. The photoemission rate is assumed to be proportional to the light intensity, and therefore the output of each photon detector may be described by a compound Poisson process in which the photon arrival rate varies stochastically with time. The classical light intensities calculated for the two detectors shown in Figure 1 would be identical; Hanbury Brown \& Twiss (1957) therefore conclude that although the photoemission rates for the two detectors both fluctuate, the fluctuations of these rates are perfectly correlated, and this leads to a nonzero correlation of the detector outputs. A similar semiclassical approach involving a compound Poisson process was described by Mandel (1959). Section 5 presents a semiclassical analysis of the sensitivity of a shot-noise measurement scheme using a single detector; the case of multiple detectors in treated in Section 6. The conclusion of the semiclassical analysis for both cases is that the shot noise schemes cannot improve on the $\sqrt{N(1+n)}$ bunching-limited sensitivity for standard photon counting.

In addition to this historical basis, the interpretation of photon bunching in terms of a time-variable photon arrival rate is both supported by experiment and fully consistent with the predictions of quantum mechanics. The fact that the photon arrival rate for thermal radiation does indeed vary with time was directly demonstrated in the laboratory by Morgan \& Mandel (1966) through measurement of the correlation of the arrival times of individual photons at a single detector. Indeed, Morgan \& Mandel (1966) give a simple, concise description of the photon bunching effect: "In time intervals of order or less than the coherence time of the light, the probability of counting two pulses is greater than that expected for random events," just as depicted in Figure 5. Furthermore, the results of the semiclassical analysis which invokes a stochastic, time-variable photon arrival rate are reproduced by a full quantum calculation. Kelley \& Kleiner (1964) describe a quantummechanical theory of photon detection that uses a density matrix to describe the state of the electromagnetic field; the HBT photon bunching effect may be studied through use of a density matrix appropriate for thermal radiation. A fully quantum analysis for shot-noise measurements using a single detector is described in Section 7, and agrees with the semiclassical results in Section 5. As discussed in Section 7, the extension of the fully quantum analysis to the case of two detectors is straightforward and agrees with the corresponding semiclassical analysis in Section 6. Thus, both the semiclassical and fully quantum calculations show that the bunching noise cannot be evaded through use of a shot noise measurement scheme, whether one uses one or two detectors. This agreement is a reflection of the equivalence of the quantum and semiclassical descriptions of light as shown by Sudarshan (1963), who made use of the coherent state representation introduced by Glauber (1963). In Appendix F, the equivalence of the quantum and semiclassical (i.e., compound Poisson) descriptions of the photocurrent statistics is demonstrated explicitly. Thus, the interpretation of photon bunching in terms of a time-varying photon arrival rate as illustrated in Figure 5 is in fact predicted by the full quantum theory and also supported by laboratory measurements.

A potentially confusing aspect of the discussion is the fact that the shot noise spectrum is white-indeed, the output spectrum for the two-detector case (Figure 4) is flat and featureless. Where is the bunching noise hiding? The answer is simple: the shot noise spectrum is white regardless of whether the photon arrival rate is constant or if it varies randomly with time due to bunching, as can easily be understood. The variation of shot noise intensity due to a randomly varying event rate is similar to that of steady shot noise subjected to a random amplitude modulation (AM). The effect of AM on a sinusoidal carrier is very well known to radio engineers: modulation sidebands are produced below and above the carrier frequency. Mathematically, a carrier at frequency $\nu_{\mathrm{c}}$ that is AM-modulated at frequency $\nu_{\mathrm{m}}$ develops sidebands at $\nu_{ \pm}=\nu_{\mathrm{c}} \pm \nu_{\mathrm{m}}$ :

$$
\begin{aligned}
& \cos \left(2 \pi \nu_{\mathrm{c}} t\right)\left[1+a \cos \left(2 \pi \nu_{\mathrm{m}} t\right)\right] \\
& \quad=\cos \left(2 \pi \nu_{\mathrm{c}} t\right)+\frac{a}{2} \cos \left(2 \pi \nu_{+} t\right)+\frac{a}{2} \cos \left(2 \pi \nu \_t\right) .
\end{aligned}
$$

This result is easily generalized to a Fourier superposition of modulation frequencies. Thus, the bunching noise component illustrated in Figure 3 may be interpreted as the sidebands on a DC carrier - the mean photocurrent - that are produced by the random modulation of the photon arrival rate. Indeed, these sidebands extend out to $\pm \Delta \nu$ which corresponds to the bandwidth of the arrival rate (or light intensity) fluctuations. Similarly, a Fourier component of shot noise at some frequency $\nu_{1}$ will develop sidebands extending over $\nu_{1} \pm \Delta \nu$ as a result of the arrival rate fluctuations. Because all Fourier components of shot noise develop these sidebands in the same way, it is clear that the net result must be a white spectrum. However, the sideband generation process introduces the possibility that the Fourier components of shot noise at different frequencies are correlated. Shot noise with a variable event rate arises in other contexts and is well studied, e.g., in the theory of diode mixers (Held \& Kerr 1978) or in the detection of fast optical pulse trains (Quinlan et al. 2013). For these examples, the event rate modulation is deterministic and periodic and the correlations between different Fourier components of shot noise play an essential role. However, these correlations vanish for the present case of photon bunching because the event rate varies randomly rather than deterministically. Indeed, the photon shot noise must be a stationary process, in the sense that all statistics such as the autocorrelation function $\langle I(t) I(t+\tau)\rangle$ are invariant under time translation $t \rightarrow t+t_{1}$, because the time-varying photon arrival rate is also a stationary random process. A translation in time changes the phase of a product of two different Fourier components, $\hat{I}^{*}(\nu) \hat{I}\left(\nu^{\prime}\right) \rightarrow$ $\hat{I}^{*}(\nu) \hat{I}\left(\nu^{\prime}\right) e^{-i 2 \pi\left(\nu-\nu^{\prime}\right) t_{1}}$, and therefore time-translation symmetry requires that the correlation of different Fourier components vanish. A more detailed mathematical proof of these statements is given in Section 4, culminating with Equation (56), which agrees with previous work (Picinbono et al. 1970). Thus, the fact that the shot noise spectrum is white tells us nothing about possible time-dependent variations of the shot noise intensity. So where is the bunching noise hiding? To find it, we must go beyond the noise spectrum, which relates to the second order statistics of the photocurrent, and look at the fourth-order photocurrent statistics that are needed to describe the fluctuations of the shot noise intensity. This calculation is presented in Section 5, leading to Equation (78), which reveals the presence of the bunching noise in the shot noise intensity. 


\section{PHOTOCURRENT SPECTRUM FOR A SINGLE DETECTOR}

I now turn to a straightforward quantum-mechanical calculation of the output noise spectrum of a detector that is illuminated with filtered thermal radiation with bandwidth $\Delta \nu$ and occupation number $n$, as in the setup shown in Figure 2. The treatment uses a conventional quantum formalism described in Zmuidzinas (2003); the calculations presented in this section are fairly standard and mainly serve to introduce the formalism and notation. The principal result, stated below in Equation (20) and illustrated in Figure 3, shows that the spectrum consists of three components: (1) a DC term corresponding to the average output; (2) a component due to photon bunching that is confined to a bandwidth equal to the optical bandwidth $\Delta \nu$; and (3) a white-noise component due to photon shot noise that is limited only by the detector output bandwidth $\nu_{\mathrm{d}}$. For conventional photon counting, the observable quantity is the time integral of the photocurrent, ${ }^{4}$ which makes use of the fact that the DC photocurrent is proportional to the mean photon rate $\bar{\Gamma}=n \Delta \nu$. For the alternative shot noise measurement technique, the observable is the time integral of the noise intensity in the white-noise region $\Delta \nu \leqslant|\nu| \leqslant \nu_{\mathrm{d}}$, and makes use of the proportionality of the shot noise intensity to the mean photon rate $\bar{\Gamma}$.

Consider an ideal photon detector illuminated by a single mode of the radiation field. The radiation field is described by photon creation and destruction operators,

$$
\begin{aligned}
b^{\dagger}(t) & =\int_{0}^{\infty} d \nu e^{-i 2 \pi \nu t} b^{\dagger}(\nu) \\
b(t) & =\int_{0}^{\infty} d \nu e^{+i 2 \pi \nu t} b(\nu)
\end{aligned}
$$

which are defined only for positive frequencies and obey Bosonic commutation relations

$$
\left[b(\nu), b^{\dagger}\left(\nu^{\prime}\right)\right]=\delta\left(\nu-\nu^{\prime}\right)
$$

I assume that the radiation field is in a thermal state described by the density matrix

$$
\rho=\exp \left(\int_{0}^{\infty} d \nu\left\{-x(\nu) b^{\dagger}(\nu) b(\nu)+\ln \left[1-e^{-x(\nu)}\right]\right\}\right) .
$$

This is of the standard form for thermal equilibrium, $\rho \propto \exp (-H / k T)$, where the Hamiltonian consists of a sum of harmonic oscillators,

$$
H=\int_{0}^{\infty} d \nu h \nu b^{\dagger}(\nu) b(\nu),
$$

and where $x(\nu)=h \nu / k T$ is the normalized inverse temperature. The $\ln \left[1-e^{-x(\nu)}\right]$ term provides the required normalization $\operatorname{Tr} \rho=1$. It is readily shown (Zmuidzinas 2003) that

\footnotetext{
4 The time integral of the photocurrent is a useful and analytically tractable quantity for quantifying the performance of ideal detectors but is not the optimal statistic for real detectors that have additional non-ideal sources of noise, e.g., amplifier noise. For example, one might use Wiener filtering followed by peak detection to locate and count the individual photon pulses in the output timestream; this approach rejects most of the noise emanating from the detection system during the time intervals between photon events.
}

this density matrix gives expectation values of

$$
\left\langle b^{\dagger}(\nu) b\left(\nu^{\prime}\right)\right\rangle=\operatorname{Tr}\left[\rho b^{\dagger}(\nu) b\left(\nu^{\prime}\right)\right]=n(\nu) \delta\left(\nu-\nu^{\prime}\right)
$$

where the occupation number is given by the Bose-Einstein formula

$$
n(\nu)=\frac{1}{e^{x(\nu)}-1}=\frac{1}{e^{h \nu / k T}-1} .
$$

Note that the excitation temperature need not be the same for all frequencies; we may easily generalize to $x(\nu)=h \nu / k T(\nu)$.

An ideal photodetector produces one pulse at its output for every photon absorbed. For example, in a superconductorinsulator-superconductor (SIS) tunnel junction detector (Tucker $\&$ Millea 1978), each absorbed photon causes one electron to tunnel across the junction. Such a detector may be described by a Hermitian photocurrent operator

$$
I_{F}(t)=\int_{-\infty}^{t} d t^{\prime} F\left(t-t^{\prime}\right) b^{\dagger}\left(t^{\prime}\right) b\left(t^{\prime}\right)
$$

where $F(t)$ describes the shape of the current pulse produced by one photon. The detector output need not be an electrical current. More generally, we can consider $I_{F}(t)$ to be the output signal of the detector when illuminated by the radiation field, and $F(t)$ to be the output signal produced when a single photon is absorbed; however I will continue to call $I_{F}(t)$ the photocurrent operator. Note that I am assuming that the detector is operating in a linear regime: doubling the photon absorption rate doubles the output signal. If the detector response is fast relative to the timescales of interest, we may approximate

$$
F(t) \approx \delta(t),
$$

and therefore consider the Hermitian operator

$$
I(t)=b^{\dagger}(t) b(t)
$$

where now $I(t)$ has units of $\mathrm{s}^{-1}$.

The impact of photon bunching on the sensitivity of measurements performed using conventional photon counting may be demonstrated by considering the operator

$$
N_{T}=\int_{-T / 2}^{T / 2} d t I(t),
$$

which represents the number of photons detected in a measurement time interval $[-T / 2, T / 2]$. The mean and variance of this operator are given in Equations (41) and (42) of Zmuidzinas (2003):

$$
\begin{gathered}
\left\langle N_{T}\right\rangle=\operatorname{Tr}\left(\rho N_{T}\right)=T \int_{0}^{\infty} d \nu n(\nu) \\
\sigma_{N_{T}}^{2}=\left\langle N_{T}^{2}\right\rangle-\left\langle N_{T}\right\rangle^{2}=T \int_{0}^{\infty} d \nu n(\nu)(1+n(\nu)) .
\end{gathered}
$$

Thus, the fractional measurement uncertainty is

$$
\frac{\sigma_{N_{T}}}{\left\langle N_{T}\right\rangle}=\frac{\sqrt{1+\tilde{n}}}{\sqrt{\left\langle N_{T}\right\rangle}}
$$

and is degraded by $\sqrt{1+\tilde{n}}$ due to photon bunching as compared to the fractional Poisson uncertainty of $1 / \sqrt{\left\langle N_{T}\right\rangle}$. 
Here the effective occupation number $\tilde{n}$ is defined by

$$
\tilde{n}=\frac{\int_{0}^{\infty} d \nu[n(\nu)]^{2}}{\int_{0}^{\infty} d \nu n(\nu)}
$$

For the simple case that $n(\nu)=n$ inside an optical bandwidth $\Delta \nu$ and zero outside, one readily finds $\tilde{n}=n$.

The calculation of the output noise spectrum of the detector makes use of the Fourier transform of the photocurrent operator:

$$
\begin{aligned}
\hat{I}(\nu)=\hat{I}^{\dagger}(-\nu)= & \int_{-\infty}^{+\infty} d t e^{-i 2 \pi \nu t} I(t) \\
= & \int_{-\infty}^{+\infty} d t e^{-i 2 \pi \nu t} b^{\dagger}(t) b(t) \\
= & \int_{0}^{\infty} d \nu_{1} \int_{0}^{\infty} d \nu_{2} b^{\dagger}\left(\nu_{1}\right) b\left(\nu_{2}\right) \\
& \times \int_{-\infty}^{+\infty} d t e^{+i 2 \pi \nu_{1} t} e^{-i 2 \pi \nu_{2} t} e^{-i 2 \pi \nu t} \\
= & \int_{0}^{\infty} d \nu_{1} b^{\dagger}\left(\nu_{1}+\nu\right) b\left(\nu_{1}\right) \theta\left(\nu_{1}+\nu\right) .
\end{aligned}
$$

The photon operators $b$ and $b^{\dagger}$ are only defined for positive frequencies; the unit step function $\theta\left(\nu_{1}+\nu\right)$ is needed to guarantee $\nu_{1}+\nu \geqslant 0$ even when $\nu<0$. For brevity of notation, I will not write the step function explicitly but instead rely on the interpretation $b\left(\nu_{1}\right) \rightarrow b\left(\nu_{1}\right) \theta\left(\nu_{1}\right)$ and similarly for $b^{\dagger}\left(\nu_{1}\right)$. Clearly, the effect of a finite pulse width $F(t)$ will be multiplication by the corresponding frequency-domain filter $\hat{F}(\nu)$,

$$
\hat{I_{F}}(\nu)=\int_{-\infty}^{+\infty} d t e^{-i 2 \pi \nu t} I_{F}(t)=\hat{F}(\nu) \hat{I}(\nu) .
$$

The power spectrum $S_{I}(\nu)=S_{I}(-\nu)$ of the photocurrent is defined by

$$
\left\langle\hat{I}^{\dagger}(\nu) \hat{I}\left(\nu^{\prime}\right)\right\rangle=S_{I}(\nu) \delta\left(\nu-\nu^{\prime}\right)
$$

including the pulse shape $F(t)$ would lead to a filtered power spectrum

$$
S_{I_{F}}(\nu)=|F(\nu)|^{2} S_{I}(\nu) .
$$

Because the thermal density matrix is Gaussian, the required expectation value may be found by combining the photon operators pairwise,

$$
\begin{aligned}
\left\langle\hat{I}^{\dagger}(\nu) \hat{I}\left(\nu^{\prime}\right)\right\rangle= & \int_{0}^{\infty} d \nu_{1} \int_{0}^{\infty} d \nu_{2}\left\langle b^{\dagger}\left(\nu_{1}\right) b\left(\nu_{1}+\nu\right)\right. \\
& \left.\times b^{\dagger}\left(\nu_{2}+\nu^{\prime}\right) b\left(\nu_{2}\right)\right\rangle \\
= & \int_{0}^{\infty} d \nu_{1} \int_{0}^{\infty} d \nu_{2}\left\langle b^{\dagger}\left(\nu_{1}\right) b\left(\nu_{1}+\nu\right)\right\rangle \\
& \times\left\langle b^{\dagger}\left(\nu_{2}+\nu^{\prime}\right) b\left(\nu_{2}\right)\right\rangle \\
& +\left\langle b^{\dagger}\left(\nu_{1}\right) b\left(\nu_{2}\right)\right\rangle\left\langle b\left(\nu_{1}+\nu\right) b^{\dagger}\left(\nu_{2}+\nu^{\prime}\right)\right\rangle \\
= & \int_{0}^{\infty} d \nu_{1} \int_{0}^{\infty} d \nu_{2}\left\{n\left(\nu_{1}\right) n\left(\nu_{2}\right) \delta(\nu)\right. \\
& +n\left(\nu_{1}\right)\left[n\left(\nu_{1}+\nu\right)+1\right] \\
& \left.\times \delta\left(\nu_{1}-\nu_{2}\right)\right\} \delta\left(\nu-\nu^{\prime}\right) .
\end{aligned}
$$

The photocurrent power spectrum is therefore given by a sum of three terms,

$$
S_{I}(\nu)=\bar{\Gamma}^{2} \delta(\nu)+\bar{\Gamma}+\int_{0}^{\infty} d \nu_{1} n\left(\nu_{1}\right) n\left(\nu_{1}+\nu\right),
$$

where, according to Equation (11), the mean photon arrival rate is

$$
\bar{\Gamma}=\int_{0}^{\infty} d \nu_{1} n\left(\nu_{1}\right)
$$

When $n(\nu)$ is constant within a bandpass $\Delta \nu$ and zero outside, we obtain $\bar{\Gamma}=n \Delta \nu$, so the occupation number $n$ may be interpreted as the number of photons per second per Hertz of optical spectrum.

The first term in $S_{I}(\nu)$, proportional to $\delta(\nu)$, represents the contribution to the power spectrum from the DC value of the photocurrent. At nonzero frequencies, only the second and third term contribute. The second term, $\bar{\Gamma}$, is white noise independent of frequency $\nu$, and represents photon shot noise. The third term is due to photon bunching, and is not white. Indeed, for a rectangular optical bandpass of width $\Delta \nu$, the spectrum of the bunching term has a triangular shape that is symmetric with respect to $\nu=0$ and extends over $-\Delta \nu \leqslant \nu \leqslant+\Delta \nu$ :

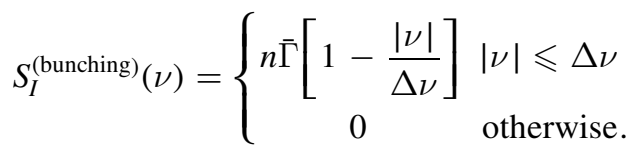

The sum of these three terms is plotted in Figure 3. At high frequencies $|\nu|>\Delta \nu$, only the white photon shot noise term contributes. This suggests the following idea: place a high-pass filter $\hat{W}(\nu)$ at the detector output that transmits only at frequencies $|\nu|>\Delta \nu$. The spectral density of the shot noise is $\bar{\Gamma}$; we can therefore measure the photon rate by measuring the noise intensity at $|\nu|>\Delta \nu$. However, as we shall see, this method does not avoid the sensitivity degradation due to photon bunching.

\section{PHOTOCURRENT CROSS-SPECTRUM FOR MULTIPLE DETECTORS}

I now generalize the discussion of Section 2 to the case of multiple detectors, in order to analyze the two-detector scheme illustrated in Figure 1, or its radio-frequency equivalent shown in Figure 6. The detection scheme proposed by Lieu et al. (2015) uses an identical two-detector setup. I start with a more general case in which an arbitrary passive linear optical system is used to illuminate a set of detectors, and calculate the crossspectral density of the output currents. The principal result (Equation (31)) is a straightforward generalization of Equation (20) for a single detector; to my knowledge this is a new result. Following Zmuidzinas (2003), the optical system is represented by a passive linear $N$-port network with a scattering matrix $S$; a $50 / 50$ beamsplitter is an example of a four-port network. The network is illuminated by incoming radiation described by the photon operators $a_{i}(\nu)$, and produces outgoing radiation according to the scattering equation

$$
b_{i}(\nu)=\sum_{j} S_{i j}(\nu) a_{i}(\nu)+c_{i}(\nu)
$$




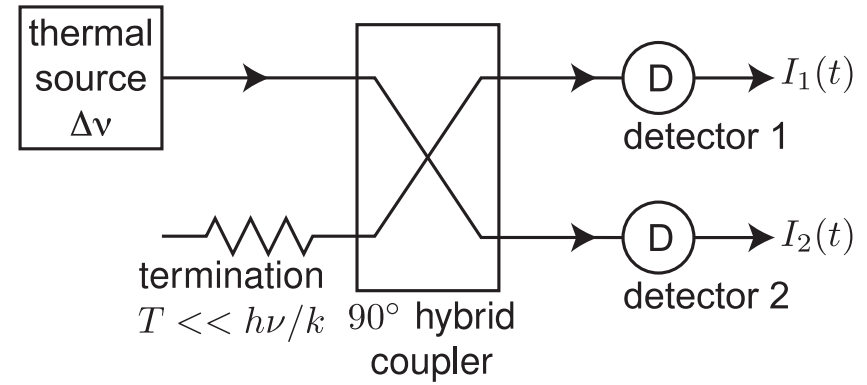

Figure 6. Radio-frequency equivalent of the two-detector plus beamsplitter setup shown in Figure 1. Free-space propagation is replaced by guided-wave propagation in transmission lines or waveguides, the function of the beamsplitter is performed by a $90^{\circ} 3 \mathrm{~dB}$ hybrid coupler (Pozar 2012), and the unused port of the coupler is connected to a cold termination. As shown by Equation (41), the spectrum of the difference of the photocurrents $I_{\Delta}(t)=I_{1}(t)-I_{2}(t)$ does not contain the DC or bunching noise components, and is therefore white across the full detector output bandwidth.

where the $c_{i}(\nu)$ are operators representing noise added by the network. Here the indices $1 \leqslant i, j \leqslant N$ label the ports of the network. The noise operators satisfy commutation relations

$$
\left[c_{i}(\nu), c_{j}\left(\nu^{\prime}\right)\right]=\left[1-S(\nu) S^{\dagger}(\nu)\right]_{j i} \delta\left(\nu-\nu^{\prime}\right),
$$

as required to preserve the Bosonic commutation relations for the output operators,

$$
\left[b_{i}(\nu), b_{j}^{\dagger}\left(\nu^{\prime}\right)\right]=\delta_{i j} \delta\left(\nu-\nu^{\prime}\right),
$$

given that the input operators also satisfy the same commutation relations,

$$
\left[a_{i}(\nu), a_{j}^{\dagger}\left(\nu^{\prime}\right)\right]=\delta_{i j} \delta\left(\nu-\nu^{\prime}\right) .
$$

If the input radiation is thermal and the $N$-port is passive, the output radiation is also thermal and may be fully described by a mode occupation matrix $B_{i j}(\nu)$, defined through

$$
\left\langle b_{i}(\nu) b_{j}^{\dagger}\left(\nu^{\prime}\right)\right\rangle=B_{i j}(\nu) \delta\left(\nu-\nu^{\prime}\right),
$$

which is a generalization of the mode occupation number $n(\nu)$. Photocurrent operators and their Fourier transforms may be introduced for each port:

$$
\begin{gathered}
I_{i}(t)=b_{i}^{\dagger}(t) b_{i}(t) . \\
\hat{I}_{i}(\nu)=\int_{0}^{\infty} d \nu_{1} b_{i}^{\dagger}\left(\nu_{1}+\nu\right) b_{i}\left(\nu_{1}\right) .
\end{gathered}
$$

The photocurrent power cross-spectrum $C_{i j}(\nu)$ is defined through the expression

$$
\left\langle\hat{I}_{i}^{\dagger}(\nu) \hat{I}_{j}\left(\nu^{\prime}\right)\right\rangle=C_{i j}(\nu) \delta\left(\nu-\nu^{\prime}\right),
$$

which I calculate using pairwise evaluation of the resulting fourth-order moments of the photon operators, as for Equation (19). The power cross-spectrum is found to be

$$
C_{i j}(\nu)=\bar{\Gamma}_{i} \bar{\Gamma}_{j} \delta(\nu)+\bar{\Gamma}_{i} \delta_{i j}+\int_{0}^{\infty} d \nu_{1} B_{i j}\left(\nu_{1}\right) B_{j i}\left(\nu_{1}+\nu\right),
$$

where the mean photon rates at the detectors are

$$
\bar{\Gamma}_{i}=\int_{0}^{\infty} d \nu_{1} B_{i i}\left(\nu_{1}\right)
$$

As was found for the single detector case and illustrated in Figure 3, we see that the power cross-spectrum $C_{i j}(\nu)$ for multiple detectors consists of three terms: a DC term $\left(\bar{\Gamma}_{i} \bar{\Gamma}_{j} \delta(\nu)\right)$, a white spectrum from photon shot noise that is uncorrelated between detectors $\left(\bar{\Gamma}_{i} \delta_{i j}\right)$, and a bunching spectrum

$$
C_{i j}^{\text {(bunching) }}(\nu)=\int_{0}^{\infty} d \nu_{1} B_{i j}\left(\nu_{1}\right) B_{j i}\left(\nu_{1}+\nu\right)
$$

that exhibits correlations between detectors but is limited to frequencies $\nu \leqslant \Delta \nu$.

We now specialize to a four-port network appropriate for a beamsplitter or $90^{\circ} 3 \mathrm{~dB}$ coupler (Pozar 2012), as illustrated in Figures 1 and 6 , with a scattering matrix given by

$$
S=\frac{1}{\sqrt{2}}\left[\begin{array}{llll}
0 & 0 & i & 1 \\
0 & 0 & 1 & i \\
i & 1 & 0 & 0 \\
1 & i & 0 & 0
\end{array}\right] .
$$

This matrix is reciprocal, $S^{T}=S$, as required by time-reversal symmetry, and $S$ is also unitary, $S S^{\dagger}=1$, and therefore the network is lossless. We will call ports 1 and 2 the output ports and place detectors on them, and ports 3 and 4 will serve as the input ports. The incoming fields $a_{i}(\nu)$ are assumed to be in independent thermal states described by occupation numbers $n_{i}(\nu)$. Port 4 will be illuminated with occupation number $n_{4}(\nu)$. Port 3 will be terminated in a vacuum state with zero occupation number; furthermore, the detectors are assumed to be cold and therefore do not radiate toward the beamsplitter, so $n_{1}(\nu)=n_{2}(\nu)=n_{3}(\nu)=0$. We may now calculate

$$
\begin{aligned}
B_{i j}(\nu) & =\sum_{k} S_{i k}(\nu) S_{j k}^{*}(\nu) n_{k}(\nu) . \\
& =S_{i 4}(\nu) S_{j 4}^{*}(\nu) n_{4}(\nu) .
\end{aligned}
$$

Therefore,

$$
B_{11}(\nu)=B_{22}(\nu)=\frac{n_{4}(\nu)}{2}
$$

while

$$
B_{12}(\nu)=B_{21}^{*}(\nu)=-i \frac{n_{4}(\nu)}{2} .
$$

Thus, the bunching power cross-spectrum for the detector ports 1,2 is

$$
C^{\text {(bunching) }}=\frac{1}{4}\left[\begin{array}{ll}
1 & 1 \\
1 & 1
\end{array}\right] \int_{0}^{\infty} d \nu_{1} n_{4}\left(\nu_{1}\right) n_{4}\left(\nu_{1}+\nu\right) ;
$$

the bunching noise is fully correlated between the detectors. The matrix in this expression has eigenvalues of 2 and 0 for the symmetric and antisymetric eigenvectors $(1,1)$ and $(1,-1)$. We therefore see that the bunching noise term will be absent for the difference of the two detector photocurrents, $I_{\Delta}(t)=I_{1}(t)-I_{2}(t)$. Neglecting the DC term, the noise matrix 
is

$$
\begin{aligned}
C(\nu)= & \frac{1}{2}\left[\begin{array}{ll}
1 & 0 \\
0 & 1
\end{array}\right] \int_{0}^{\infty} d \nu_{1} n_{4}\left(\nu_{1}\right) \\
& +\frac{1}{4}\left[\begin{array}{ll}
1 & 1 \\
1 & 1
\end{array}\right] \int_{0}^{\infty} d \nu_{1} n_{4}\left(\nu_{1}\right) n_{4}\left(\nu_{1}+\nu\right)
\end{aligned}
$$

where the first term represents the shot noise, which is uncorrelated between detectors. This is the only term that remains when we calculate the spectral density of $I_{\Delta}$,

$$
\begin{aligned}
C_{\Delta}(\nu) & =\left[\begin{array}{ll}
1 & -1
\end{array}\right] C(\nu)\left[\begin{array}{c}
1 \\
-1
\end{array}\right] \\
& =\int_{0}^{\infty} d \nu_{1} n_{4}\left(\nu_{1}\right)=\bar{\Gamma},
\end{aligned}
$$

which is the same as the shot noise intensity for a single detector without the beamsplitter. To summarize, the difference of the two detector currents $I_{\Delta}(t)=I_{1}(t)-I_{2}(t)$ has a white spectrum, given by

$$
\left\langle\hat{I}_{\Delta}^{\dagger}(\nu) \hat{I}_{\Delta}\left(\nu^{\prime}\right)\right\rangle=\bar{\Gamma} \delta\left(\nu-\nu^{\prime}\right)
$$

and illustrated in Figure 4.

\section{SPECTRUM OF SHOT NOISE WITH A VARIABLE EVENT RATE}

As discussed in Section 1, in the semiclassical picture one views photon bunching as being caused by a stochastically varying photon arrival rate. Here I calculate the spectrum of classical shot noise for a time-varying event rate and demonstrate that the shot noise remains white and uncorrelated, as discussed qualitatively in Section 1. I further demonstrate that the output noise spectrum of a single detector calculated in the semiclassical picture can reproduce the quantum-mechanical result given in Section 2. The principal result is given by Equation (56), which reproduces an earlier result of Picinbono et al. (1970); the purpose of presenting the detailed derivation here is to introduce the formalism in preparation for the calculation of shot noise intensity fluctuations in Section 5 .

A classical current containing shot noise, e.g., the current across a tunnel barrier with a low transmission probability, may be considered to be a sum of impulses,

$$
I(t)=\sum_{i} \delta\left(t-t_{i}\right)
$$

where $\left\{t_{i}\right\}$ represent the times at which discrete charges (e.g., electrons) jump across the barrier. To remain consistent with Sections 2 and 3, I have omitted the usual factor of electron charge $e$, so $I(t)$ has units of $\mathrm{s}^{-1}$ or $\mathrm{Hz}$. Suppose further that the average current is time-dependent,

$$
\langle I(t)\rangle=\Gamma(t)=\bar{\Gamma}+\delta \Gamma(t),
$$

where $\bar{\Gamma}$ is the mean event rate and $\delta \Gamma(t)$ represents variations in the rate and therefore has zero mean. If the event rate $\Gamma(t)$ is constant, i.e., $\delta \Gamma(t)=0$, we know that the current has a shot noise spectrum that is white and has intensity $\bar{\Gamma}$. On the other hand, if the event rate varies with time, we expect the shot noise intensity to also vary. Thus, a time-resolved measurement of the shot noise intensity should allow us to measure the corresponding time-dependent current. This possibility is investigated here and further in Section 5.
I follow the approach of Kelley \& Kleiner (1964) for calculating classical shot-noise statistics; a similar but mathematically more formal approach is given by Picinbono et al. (1970). Let $y_{i}$ be a random variable that represents the number of charges that flow during the time interval $\left[t_{i}, t_{i}+\Delta t_{i}\right]$. I assume that $y_{i}$ is independent of all other $y_{j}$ for $j \neq i$, and furthermore that for sufficiently small time intervals $\Delta t_{i}, y_{i}$ has a probability distribution given by

$$
\begin{aligned}
& P\left(y_{i}=1\right)=\Gamma\left(t_{i}\right) \Delta t_{i} \\
& P\left(y_{i}=0\right)=1-\Gamma\left(t_{i}\right) \Delta t_{i} .
\end{aligned}
$$

The number of charges that cross during the time interval $[0, T]$ is a random variable given by

$$
N_{T}=\int_{0}^{T} I(t) d t \approx \sum_{i=1}^{M} y_{i}
$$

where the time interval $[0, T]$ has been split into $M$ nonoverlapping subintervals $\left[t_{i}, t_{i}+\Delta t_{i}\right]$. The distribution of $N_{T}$ is encoded by the moment generating function calculated in Appendix A:

$$
\begin{aligned}
G_{N}(s) & =\left\langle e^{s N_{T}}\right\rangle=\sum_{k=0}^{\infty} \frac{s^{k}}{k !} \sum_{i_{1} \ldots i_{k}=1}^{M}\left\langle y_{i_{1}} \ldots y_{i_{k}}\right\rangle \\
& =\exp \left[\mu_{T}\left(e^{s}-1\right)\right] \\
& =e^{-\mu_{T}} \sum_{k=0}^{\infty} \frac{\mu_{T}^{k}}{k !} e^{s k}
\end{aligned}
$$

As expected (Mandel 1958), this result shows that $N_{T}$ follows a Poisson distribution with mean

$$
\mu_{T}=\int_{0}^{T} d t \Gamma(t)
$$

Thus, the current $I(t)$ is a Poisson process with a timedependent rate $\Gamma(t)$.

The same formalism can be used to work out the spectrum of shot noise for a time-dependent current. The time-limited Fourier transform of the current is defined as

$$
\hat{I}_{T}(\nu)=\int_{-T / 2}^{+T / 2} d t I(t) e^{-i 2 \pi \nu t},
$$

which allows the power spectrum to be computed by evaluating the limit

$$
\lim _{T \rightarrow \infty}\left\langle\hat{I}_{T}(\nu) \hat{I}_{T}^{*}\left(\nu^{\prime}\right)\right\rangle
$$

Expressing the current in terms of the random variables $y_{i}$ gives

$$
\hat{I}_{T}(\nu) \approx \sum_{i=1}^{M} y_{i} e^{-i 2 \pi \nu t_{i}}
$$

Therefore,

$$
\left\langle\hat{I}_{T}(\nu) \hat{I}_{T}^{*}\left(\nu^{\prime}\right)\right\rangle_{y} \approx \sum_{i, j=1}^{M}\left\langle y_{i} y_{j}\right\rangle e^{-i 2 \pi \nu t_{i}} e^{+i 2 \pi \nu^{\prime} t_{j}} .
$$

Now

$$
\left\langle y_{i} y_{j}\right\rangle=\left\langle y_{i}\right\rangle\left\langle y_{j}\right\rangle+\delta_{i j}\left(\left\langle y_{i}^{2}\right\rangle-\left\langle y_{i}\right\rangle^{2}\right)
$$




$$
=\Gamma\left(t_{i}\right) \Gamma\left(t_{j}\right) \Delta t_{i} \Delta t_{j}+\delta_{i j}\left[\Gamma\left(t_{i}\right) \Delta t_{i}-\Gamma^{2}\left(t_{i}\right)\left(\Delta t_{i}\right)^{2}\right],
$$

making use of $y_{i}^{2}=y_{i}$. Inserting the first term into the sum and taking the continuum limit gives

$$
\begin{gathered}
\lim _{T \rightarrow \infty} \int_{-T / 2}^{T / 2} d t_{1} \Gamma\left(t_{1}\right) e^{-i 2 \pi \nu t_{1}} \int_{-T / 2}^{T / 2} d t_{2} \Gamma\left(t_{1}\right) e^{+i 2 \pi \nu t_{1}} \\
\quad=(\bar{\Gamma} \delta(\nu)+\delta \hat{\Gamma}(\nu))\left(\bar{\Gamma} \delta\left(\nu^{\prime}\right)+\delta \hat{\Gamma}^{*}\left(\nu^{\prime}\right)\right)
\end{gathered}
$$

while the second term yields

$$
\bar{\Gamma} \delta\left(\nu-\nu^{\prime}\right)+\delta \hat{\Gamma}\left(\nu-\nu^{\prime}\right)
$$

note that the $\left(\Delta t_{i}\right)^{2}$ term vanishes in the continuum limit. Thus, the Fourier components of shot noise are correlated when the event rate varies deterministically with time, according to

$$
\begin{aligned}
\left\langle\hat{I}(\nu) \hat{I}^{*}\left(\nu^{\prime}\right)\right\rangle_{y}= & (\bar{\Gamma} \delta(\nu)+\delta \hat{\Gamma}(\nu))\left(\bar{\Gamma} \delta\left(\nu^{\prime}\right)+\delta \hat{\Gamma}^{*}\left(\nu^{\prime}\right)\right) \\
& +\bar{\Gamma} \delta\left(\nu-\nu^{\prime}\right)+\delta \hat{\Gamma}\left(\nu-\nu^{\prime}\right) .
\end{aligned}
$$

If the event rate has stochastic time-dependent rate variations $\delta \Gamma(t)$, taken to be a stationary random process with power spectrum $S_{\Gamma}(\nu)$, the shot noise spectrum may be obtained by averaging Equation (55) over $\delta \Gamma$ :

$$
\left\langle\hat{I}(\nu) \hat{I}^{*}\left(\nu^{\prime}\right)\right\rangle_{y, \delta \Gamma}=\left[\bar{\Gamma}^{2} \delta(\nu)+\bar{\Gamma}+S_{\Gamma}(\nu)\right] \delta\left(\nu-\nu^{\prime}\right) ;
$$

the $\delta\left(\nu-\nu^{\prime}\right)$ factor indicates that different Fourier components are uncorrelated and therefore this compound Poisson process is stationary, as expected. Specifically, the shot noise component $\bar{\Gamma} \delta\left(\nu-\nu^{\prime}\right)$ remains white and uncorrelated, as promised in Section 1. Equation (56) agrees with the result of Picinbono et al. (1970) (their Equation (2.27)), who claim agreement with an earlier result by Mandel.

The power spectrum of this classical compound Poisson process may be made identical to the spectrum of the photocurrent calculated quantum-mechanically (Equation (20)), provided we make the identifications

$$
\bar{\Gamma}=\int_{0}^{+\infty} d \nu_{1} n\left(\nu_{1}\right)
$$

and

$$
S_{\Gamma}(\nu)=\int_{0}^{+\infty} d \nu_{1} n\left(\nu_{1}\right) n\left(\nu_{1}+\nu\right) .
$$

If the occupation number $n(\nu)$ is constant across an optical bandwidth $\Delta \nu$ and zero outside, the relative importance of the bunching and Poisson terms at noise frequencies well below $\Delta \nu$ is governed by

$$
\frac{S_{\Gamma}(0)}{\bar{\Gamma}}=\frac{\int_{0}^{+\infty} d \nu_{1} n^{2}\left(\nu_{1}\right)}{\int_{0}^{+\infty} d \nu_{1} n\left(\nu_{1}\right)}=\frac{n^{2} \Delta \nu}{n \Delta \nu}=n .
$$

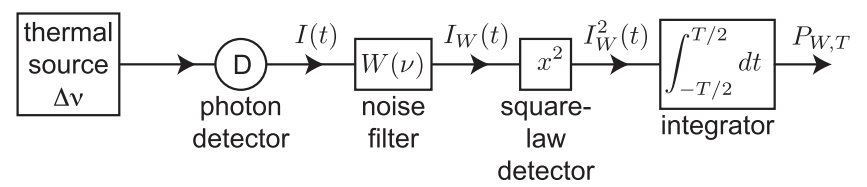

Figure 7. Signal flow diagram for shot-noise detection using a single detector. A noise filter $W(\nu)$ allows selection of the portion of the spectrum where shot noise dominates (e.g., the hatched region in Figure 3) prior to the measurement of the noise intensity using a square-law detector and integrator.

\section{SHOT NOISE MEASUREMENT: SEMICLASSICAL ANALYSIS FOR A SINGLE DETECTOR}

I now turn to the computation of the fluctuations in the intensity of classical shot noise with a time-varying event rate and demonstrate that the shot noise intensity reflects variations in the event rate. I apply these results to the case of photon detection under the assumption that bunching may be described by a stochastic photon arrival rate whose mean and power spectrum are described by Equations (57) and (58), respectively. As discussed in Section 1 and Appendix F, this assumption is consistent with the full quantum theory and is supported by experiment. The principal results presented in this section (Equations (71), (74), (75), and (78)) are new and demonstrate that shot noise measurements using a single detector suffer the same $\sqrt{1+n}$ sensitivity degradation due to photon bunching as would occur for direct photon counting.

Because the rate fluctuations $S_{\Gamma}(\nu)$ have a limited bandwidth (Equation (58)), only shot noise contributes to the current noise (Equation (56)) at high frequencies, and therefore a measurement of the intensity of the high-frequency noise should give us information on the mean rate $\bar{\Gamma}$ and therefore the mean current. Specifically, the output noise spectrum for a photon detector as illustrated in Figure 3 suggests use of the shot noise measurement setup shown in Figure 7, which includes a noise filter $W(\nu)$ for isolating the shot noise dominated portion of the spectrum. We therefore consider applying a filter to the current,

$$
I_{W}(t)=\int_{-\infty}^{T} d t_{1} W\left(t-t_{1}\right) I\left(t_{1}\right)
$$

and then continuously integrating the noise power over a measurement interval $[-T / 2, T / 2]$,

$$
\begin{aligned}
P_{W, T}= & \int_{-T / 2}^{T / 2} d t\left[I_{W}(t)\right]^{2} \\
= & \int_{-T / 2}^{T / 2} d t\left[\int_{-\infty}^{t} d t_{1} W\left(t-t_{1}\right) I\left(t_{1}\right)\right]^{2} \\
= & \left.\int_{-T / 2}^{T / 2} d t\left[\int_{-\infty}^{+\infty} d \nu e^{i 2 \pi \nu t} W(\nu)\right) \hat{I}(\nu)\right]^{2} \\
= & \int_{-\infty}^{+\infty} d \nu_{1} \int_{-\infty}^{+\infty} d \nu_{2} \hat{I}\left(\nu_{1}\right) \hat{I}^{*}\left(\nu_{2}\right) W\left(\nu_{1}\right) W^{*}\left(\nu_{2}\right) \\
& \times \int_{-T / 2}^{T / 2} d t e^{i 2 \pi \nu_{1} t} e^{-i 2 \pi \nu_{2} t} \\
\rightarrow & \int_{-\infty}^{+\infty} d \nu_{1}\left|\hat{I}\left(\nu_{1}\right)\right|^{2}\left|W\left(\nu_{1}\right)\right|^{2} \text { as } T \rightarrow \infty .
\end{aligned}
$$


The mean value of this measure of the high-frequency noise intensity is

$$
\begin{aligned}
&\left\langle P_{W, T}\right\rangle= \int_{-\infty}^{+\infty} d \nu_{1} \int_{-\infty}^{+\infty} d \nu_{2}\left\langle\hat{I}\left(\nu_{1}\right) \hat{I}^{*}\left(\nu_{2}\right)\right\rangle \\
& \times W\left(\nu_{1}\right) W^{*}\left(\nu_{2}\right) \int_{-T / 2}^{T / 2} d t e^{i 2 \pi \nu_{1} t} e^{-i 2 \pi \nu_{2} t} \\
&= \int_{-\infty}^{+\infty} d \nu_{1} S_{I}\left(\nu_{1}\right)\left|W\left(\nu_{1}\right)\right|^{2} \int_{-T / 2}^{T / 2} d t \\
&=\bar{\Gamma} T \int_{-\infty}^{+\infty} d \nu_{1}\left|W\left(\nu_{1}\right)\right|^{2}+T \int_{-\infty}^{+\infty} d \nu_{1}\left|W\left(\nu_{1}\right)\right|^{2} S_{\Gamma}\left(\nu_{1}\right)
\end{aligned}
$$

The second term picks up the bunching noise component but may be made negligible by choosing $W(\nu)$ to be zero at the lower frequencies where $S_{\Gamma}(\nu)$ has an appreciable value, as illustrated by the hatched region in Figure 3. With this choice, if the noise measurement bandwidth is defined as

$$
2 B=\int_{-\infty}^{+\infty} d \nu_{1}\left|W\left(\nu_{1}\right)\right|^{2}
$$

where the factor of two accounts for negative frequencies, we have

$$
\left\langle P_{W, T}\right\rangle=2 \bar{\Gamma} B T,
$$

and therefore the measurement scheme shown in Figure 7 provides us with the desired information on $\bar{\Gamma}$.

However, we expect that the intensity of the shot noise should be affected by the rate fluctuations $\delta \Gamma(t)$. We are therefore interested in the fluctuations

$$
\sigma_{P}^{2}=\left\langle P_{W, T}^{2}\right\rangle-\left\langle P_{W, T}\right\rangle^{2} .
$$

This quantity will require evaluation of the fourth moments of the form

$$
\left\langle\hat{I}\left(\nu_{1}\right) \hat{I}\left(\nu_{2}\right)^{*} \hat{I}\left(\nu_{3}\right) \hat{I}\left(\nu_{4}\right)^{*}\right\rangle .
$$

Because of the presence of the high-pass filter $W(\nu)$, we may safely assume that none of the frequencies are zero, and therefore omit the DC terms. Using the same approach as before, we write

$$
\begin{aligned}
& \left\langle\hat{I}\left(\nu_{1}\right) \hat{I}\left(\nu_{2}\right)^{*} \hat{I}\left(\nu_{3}\right) \hat{I}\left(\nu_{4}\right)^{*}\right\rangle_{y} \\
& \quad \approx \sum_{i j k l}\left\langle y_{i} y_{j} y_{k} y_{l}\right\rangle e^{-i 2 \pi \nu_{1} t_{i}} e^{+i 2 \pi \nu_{2} t_{j}} e^{-i 2 \pi \nu_{3} t_{k}} e^{-i 2 \pi \nu_{4} t_{l}} .
\end{aligned}
$$

Appendix B provides the details of the evaluation of this quantity, leading to an expression for $\sigma_{P}^{2}$ in the long measurement time limit $\Delta \nu T \gg 1$ involving seven terms, labeled $\mathrm{A} 1 \mathrm{~b}+\mathrm{A} 1 \mathrm{c}, \mathrm{B} 2+\mathrm{B} 3+\mathrm{B} 4+\mathrm{B} 5, \mathrm{C} 2 \mathrm{a}+\mathrm{C} 3 \mathrm{a}, \mathrm{C} 1 \mathrm{~b}, \mathrm{C} 2 \mathrm{~b}$ $+\mathrm{C} 3 \mathrm{~b}, \mathrm{C} 4 \mathrm{~b}+\mathrm{C} 5 \mathrm{~b}+\mathrm{C} 6 \mathrm{~b}+\mathrm{C} 7 \mathrm{~b}$, and D1. Three of these terms drop out if we design our filter $W(\nu)$ so that it rejects noise due to the rate fluctuations, i.e.,

$$
\int_{-\infty}^{+\infty} d \nu|W(\nu)|^{2} S_{\Gamma}(\nu) \rightarrow 0,
$$

as illustrated by the hatched region in Figure 3 . The surviving terms $(\mathrm{C} 2 \mathrm{a}+\mathrm{C} 3 \mathrm{a}, \mathrm{C} 1 \mathrm{~b}, \mathrm{C} 2 \mathrm{~b}+\mathrm{C} 3 \mathrm{~b}$, and $\mathrm{D} 1)$ contribute fractional fluctuations of

$$
\begin{aligned}
\frac{\sigma_{P}^{2}}{\left\langle P_{W, T}\right\rangle^{2}}= & \frac{1}{T}\left\{\frac{2 \int d \nu|W(\nu)|^{4}}{\left[\int d \nu|W(\nu)|^{2}\right]^{2}}+\frac{S_{\Gamma}(0)}{\bar{\Gamma}^{2}}\right. \\
& +\frac{2 \int d \nu d \nu^{\prime}|W(\nu)|^{2}\left|W\left(\nu^{\prime}\right)\right|^{2} S_{\Gamma}\left(\nu-\nu^{\prime}\right)}{\bar{\Gamma}^{2}\left[\int d \nu|W(\nu)|^{2}\right]^{2}} \\
& +\frac{1}{\bar{\Gamma}\} .}
\end{aligned}
$$

The interpretation of these terms is simplified by choosing a filter function $W(\nu)$ which is unity inside a measurement bandwidth $B$ and zero outside, so that

$$
\int_{-\infty}^{+\infty} d \nu|W(\nu)|^{2}=\int_{-\infty}^{+\infty} d \nu|W(\nu)|^{4}=2 B
$$

including contributions from positive and negative frequencies. If we define an effective bandwidth for the rate fluctuations,

$$
\Delta \nu=\frac{\int d \nu S_{\Gamma}(\nu)}{S_{\Gamma}(0)}
$$

and evaluate the third term under the assumption that a wide bandwidth is chosen in order to optimize the shot-noise measurement, $B \gg \Delta \nu$, the terms simplify to

$$
\frac{\sigma_{P}^{2}}{\left\langle P_{W, T}\right\rangle^{2}}=\frac{1}{B T}+\frac{S_{\Gamma}(0)}{\bar{\Gamma}^{2} T}+\frac{S_{\Gamma}(0) \Delta \nu}{\bar{\Gamma}^{2} B T}+\frac{1}{\bar{\Gamma} T} .
$$

The last term is due to the Poisson fluctuations in the number of events over a time $T$ that one must have even if the event rate is constant. Meanwhile, the first term represents the noise that results from measurement of a finite number of independent samples associated with the time-bandwidth product $B T$; indeed, one sees that this term reproduces Dicke's result (Dicke 1946) in the shot noise context. Thus, it is helpful to use a large shot noise measurement bandwidth, although the Poisson term dominates when the bandwidth exceeds the mean event rate, $B>\bar{\Gamma}$. The second and third term represent the effect of event rate fluctuations, being proportional to the spectral density of the fractional fluctuations $S_{\Gamma}(0) / \bar{\Gamma}^{2}$. Here the spectral density $S_{\Gamma}(\nu)$ is evaluated at zero frequency because the rate fluctuations are being averaged over a long measurement time $T$. The last three terms in Equation (74) may be rearranged to read

$$
\frac{\sigma_{P}^{2}}{\left\langle P_{W, T}\right\rangle^{2}}=\frac{1}{B T}+\frac{1}{\bar{\Gamma} T}\left[1+\frac{S_{\Gamma}(0)}{\bar{\Gamma}}+\frac{S_{\Gamma}(0)}{\bar{\Gamma}} \frac{\Delta \nu}{B}\right] .
$$

In this form, the last term in the square brackets can be seen to represent a correction to the rate fluctuation term due to finite measurement bandwidth and is negligible under the assumption $B \gg \Delta \nu$.

If we make use of the identifications appropriate for thermal photon noise given by Equations (57) and (58), and furthermore assume that the occupation number $n(\nu)$ is constant inside an optical bandwidth $\Delta \nu$ and zero outside, 


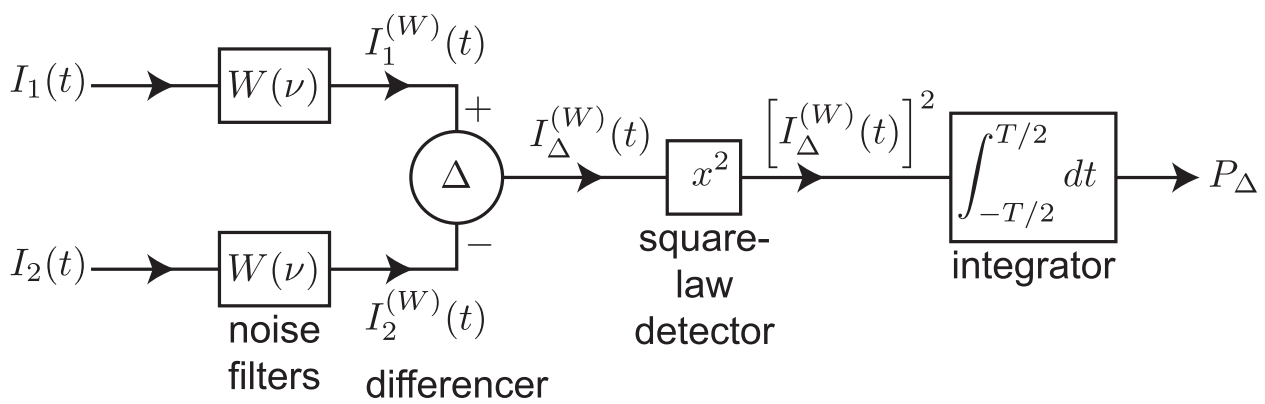

Figure 8. Signal flow diagram for shot-noise detection using two detectors. In principle, the ordering of the noise filtering and differencing operations may be interchanged since both are linear.

we have

$$
\bar{\Gamma}=n \Delta \nu
$$

and

$$
S_{\Gamma}(0)=n^{2} \Delta \nu
$$

The fractional fluctuation in the shot noise intensity is then given by

$$
\frac{\sigma_{P}}{\left\langle P_{W, T}\right\rangle}=\sqrt{\frac{1}{B T}+\frac{1+n}{\bar{\Gamma} T}}
$$

In the limit $B \gg \bar{\Gamma}$, we recover the usual result (Equation (13)) that photon bunching gives a sensitivity penalty of $\sqrt{1+n}$ as compared to Poisson statistics. This occurs despite the use of the white portion of the shot noise spectrum to measure the photon rate.

\section{SEMICLASSICAL ANALYSIS FOR MULTIPLE DETECTORS}

The extension of the semiclassical treatment in Section 5 to the case of multiple detectors is straightforward and allows us to analyze the sensitivity of shot noise measurement schemes applied to the two-detector setup proposed by Lieu et al. (2015), shown in Figures 1 and 6. The principal results (Equations (93) and (96)) are new and agree with those in Section 5; they demonstrate that shot noise measurements applied to the two-detector scheme also cannot evade the $\sqrt{1+n}$ sensitivity degradation due to photon bunching. We perform our analysis for a signal processing setup (Figure 8) similar to those typically used for experimental measurements of shot noise (Schoelkopf et al. 1997), though it differs in detail from the signal processing proposed by Lieu et al. (2015). Nonetheless, our calculations are directly applicable to the regime that Lieu et al. claim leads to suppression of the bunching noise; a detailed comparison of the calculations is given in Section 9.

Suppose we have multiple currents exhibiting shot noise,

$$
I_{a}(t)=\sum_{i} \delta\left(t-t_{i, a}\right)
$$

with time-dependent event rates

$$
\left\langle I_{a}(t)\right\rangle=\Gamma_{a}(t)=\bar{\Gamma}_{a}+\delta \Gamma_{a}(t) .
$$

Here $a$ is a discrete index that labels the currents. The rate fluctuations are stationary stochastic processes described by a cross-spectral correlation matrix,

$$
\left\langle\delta \hat{\Gamma}_{a}(\nu) \delta \hat{\Gamma}_{b}^{*}\left(\nu^{\prime}\right)\right\rangle=C_{a b}^{(\Gamma)}(\nu) \delta\left(\nu-\nu^{\prime}\right) .
$$

As before, I discretize time and introduce random variables $y_{a, i}$ to represent the number of events for current $a$ in the time interval $\left[t_{i}, \Delta t_{i}\right]$. The cross-spectral density between two currents is given by

$$
\left\langle\hat{I}_{a 1}\left(\nu_{1}\right) \hat{I}_{a 2}^{*}\left(\nu_{2}\right)\right\rangle \approx \sum_{i, j}\left\langle y_{a 1, i} y_{a 2, j}\right\rangle e^{-2 \pi \nu_{1} t_{i}} e^{+2 \pi \nu_{2} t_{j}}
$$

The $y_{a, i}$ are all independent, so

$$
\left\langle y_{a 1, i} y_{a 2, j}\right\rangle=\left\langle y_{a 1, i}\right\rangle\left\langle y_{a 2, j}\right\rangle+\delta_{i j} \delta_{a 1, a 2}\left(\left\langle y_{a 1, i}^{2}\right\rangle-\left\langle y_{a 1, i}\right\rangle^{2}\right) \text {. }
$$

The term $\left\langle y_{a 1, i}\right\rangle^{2}$ is of higher order in $\Delta t_{i}$ and can be neglected in the continuum limit:

$$
\begin{aligned}
\left\langle\hat{I}_{a 1}\left(\nu_{1}\right) \hat{I}_{a 2}^{*}\left(\nu_{2}\right)\right\rangle_{y}= & \left(\bar{\Gamma}_{a 1} \delta\left(\nu_{1}\right)+\delta \hat{\Gamma}_{a 1}\left(\nu_{1}\right)\right) \\
& \times\left(\bar{\Gamma}_{a 2} \delta\left(\nu_{2}\right)+\delta \hat{\Gamma}_{a 2}^{*}\left(\nu_{2}\right)\right) \\
& +\delta_{a 1, a 2}\left[\bar{\Gamma}_{a 1} \delta\left(\nu_{1}-\nu_{2}\right)\right. \\
& \left.+\delta \hat{\Gamma}_{a 1}\left(\nu_{1}-\nu_{2}\right)\right] .
\end{aligned}
$$

Averaging over the random process $\delta \Gamma_{a}(t)$ yields

$$
\begin{aligned}
\left\langle\hat{I}_{a 1}\left(\nu_{1}\right) \hat{I}_{a 2}^{*}\left(\nu_{2}\right)\right\rangle_{y, \delta \Gamma}= & {\left[\bar{\Gamma}_{a 1} \bar{\Gamma}_{a 2} \delta\left(\nu_{1}\right)+\delta_{a 1, a 2} \bar{\Gamma}_{a 1}\right.} \\
& \left.+C_{a 1, a 2}^{(\Gamma)}\left(\nu_{1}\right)\right] \delta\left(\nu_{1}-\nu_{2}\right),
\end{aligned}
$$

which is simply a generalization of the single-detector result given in Equation (56). If we compare this result to Equation (31) for the photocurrent correlations among detectors illuminated with thermal radiation, we see that the expressions coincide if we make the identifications

$$
\bar{\Gamma}_{a}=\bar{I}_{a}=\int_{0}^{\infty} d \nu B_{a a}(\nu)
$$

and

$$
C_{a b}^{(\Gamma)}(\nu)=\int_{0}^{\infty} d \nu^{\prime} B_{a b}\left(\nu^{\prime}\right) B_{b a}\left(\nu^{\prime}+\nu\right),
$$

which are generalizations of Equations (57) and (58).

The shot-noise measurement scheme for a single detector shown in Figure 7 may easily be adapted for use with two 
detectors as shown in Figure 8; this setup is designed to measure the shot noise intensity in the difference of the two currents, $I_{\Delta}=I_{1}-I_{2}$, as proposed by Lieu et al. (2015). Although the filter $W(\nu)$ is no longer needed for rejection of the bunching noise at low frequencies, it is maintained in the setup since any real system has a finite bandwidth. The output of the shot noise intensity measurement is given by

$$
\begin{aligned}
P_{\Delta}= & \int_{-T / 2}^{T / 2} d t\left\{\int_{-\infty}^{t} d t_{1} W\left(t-t_{1}\right)\left[I_{1}\left(t_{1}\right)-I_{2}\left(t_{1}\right)\right]\right\}^{2} \\
= & \int_{-\infty}^{+\infty} d \nu_{1} \int_{-\infty}^{+\infty} d \nu_{2}\left[\hat{I}_{1}\left(\nu_{1}\right) \hat{I}_{1}^{*}\left(\nu_{2}\right)\right. \\
& \left.+\hat{I}_{2}\left(\nu_{1}\right) \hat{I}_{2}^{*}\left(\nu_{2}\right)-2 \hat{I}_{1}\left(\nu_{1}\right) \hat{I}_{2}^{*}\left(\nu_{2}\right)\right] \\
& \times W\left(\nu_{1}\right) W^{*}\left(\nu_{2}\right) \int_{-T / 2}^{T / 2} d t e^{i 2 \pi \nu_{1} t} e^{-i 2 \pi \nu_{2} t}
\end{aligned}
$$

and has an average value

$$
\begin{aligned}
\left\langle P_{\Delta}\right\rangle= & T \int_{-\infty}^{+\infty} d \nu|W(\nu)|^{2}\left[\bar{\Gamma}_{1}+C_{11}^{(\Gamma)}(\nu)\right. \\
& \left.+\bar{\Gamma}_{2}+C_{22}^{(\Gamma)}(\nu)-2 C_{12}^{(\Gamma)}(\nu)\right] .
\end{aligned}
$$

Equations (38) and (87) give

$$
\begin{aligned}
C_{11}^{(\Gamma)}(\nu) & =C_{22}^{(\Gamma)}(\nu)=C_{12}^{(\Gamma)}(\nu) \\
& =\frac{1}{4} \int_{0}^{\infty} d \nu^{\prime} n_{4}\left(\nu^{\prime}\right) n_{4}\left(\nu^{\prime}+\nu\right)
\end{aligned}
$$

and therefore

$$
\left\langle P_{\Delta}\right\rangle=2 B T\left(\bar{\Gamma}_{1}+\bar{\Gamma}_{2}\right)
$$

is a measure of the total event rate regardless of the choice of the filter passband $W(\nu)$.

Calculation of the sensitivity of this shot-noise intensity measurement requires evaluation of fourth-order moments of the photocurrent,

$$
\begin{aligned}
F_{a b c d}= & \left\langle I_{a}^{(W)} I_{b}^{(W)} I_{c}^{(W)} I_{d}^{(W)}\right\rangle \\
= & \int_{-\infty}^{+\infty} d \nu_{1} d \nu_{2} d \nu_{3} d \nu_{4}\left\langle\hat{I}_{a}\left(\nu_{1}\right) \hat{I}_{b}^{*}\left(\nu_{2}\right) \hat{I}_{c}\left(\nu_{3}\right) \hat{I}_{d}^{*}\left(\nu_{4}\right)\right\rangle \\
& \times W\left(\nu_{1}\right) W^{*}\left(\nu_{2}\right) W\left(\nu_{3}\right) W^{*}\left(\nu_{4}\right) \\
& \times \int_{-T / 2}^{T / 2} d t e^{i 2 \pi \nu_{1} t} e^{-i 2 \pi \nu_{2} t} \int_{-T / 2}^{T / 2} d t^{\prime} e^{i 2 \pi \nu_{3} t^{\prime}} e^{-i 2 \pi \nu_{4} t^{\prime}} .
\end{aligned}
$$

This expression may be evaluated using the same approach as used for the second moment; the details are given in Appendix C. The resulting variance of the shot noise intensity is derived at the end of the appendix:

$$
\begin{aligned}
\sigma_{P_{\Delta}}^{2}= & \left\langle P_{\Delta}^{2}\right\rangle-\left\langle P_{\Delta}\right\rangle^{2}=2 F_{1111}+2 F_{1122}+4 F_{1212}-8 F_{1112} \\
= & +8 T \bar{\Gamma}_{1}^{2} \int d \nu|W(\nu)|^{4}+4 T C_{11}^{(\Gamma)}(0)\left[\int d \nu|W(\nu)|^{2}\right]^{2} \\
& +8 T \int d \nu d \nu^{\prime}|W(\nu)|^{2}\left|W\left(\nu^{\prime}\right)\right|^{2} C_{11}^{(\Gamma)}\left(\nu-\nu^{\prime}\right) \\
& +2 T \bar{\Gamma}_{1}\left[\int d \nu|W(\nu)|^{2}\right]^{2}
\end{aligned}
$$

To compare to the previous single-detector case (Equation (74)), we make the substitutions

$$
\bar{\Gamma}_{1}=\frac{1}{2} \bar{\Gamma}
$$

and

$$
C_{11}^{(\Gamma)}(\nu)=\frac{1}{4} S_{\Gamma}(\nu) .
$$

Using the mean value of $P_{\Delta}$ given by Equation (89), we may express the fractional fluctuation in the noise intensity of the difference current as

$$
\begin{aligned}
\frac{\sigma_{P_{\Delta}}^{2}}{\left\langle P_{\Delta}\right\rangle^{2}}= & \frac{1}{T}\left[\frac{2 \int d \nu|W(\nu)|^{4}}{\left[\int d \nu|W(\nu)|^{2}\right]^{2}}+\frac{S_{\Gamma}(0)}{\bar{\Gamma}^{2}}\right. \\
& +\frac{2 \int d \nu d \nu^{\prime}|W(\nu)|^{2}\left|W\left(\nu^{\prime}\right)\right|^{2} S_{\Gamma}\left(\nu-\nu^{\prime}\right)}{\bar{\Gamma}^{2}\left[\int d \nu|W(\nu)|^{2}\right]^{2}} \\
& \left.+\frac{1}{\bar{\Gamma}}\right],
\end{aligned}
$$

which is exactly our previous result for a single detector given by Equation (71). In particular, the rate fluctuation term $S_{\Gamma}(0)$ leads to the $\sqrt{1+n}$ sensitivity degradation due to photon bunching. However, it is no longer necessary to make the assumption that the filter $W(\nu)$ rejects the low-frequency excess noise; differencing the two detectors fed by a 50/50 beamsplitter takes care of the rejection instead.

\section{SHOT NOISE MEASUREMENT: A QUANTUM CALCULATION}

The semiclassical analyses given in Sections 5 and 6 for shot noise measurements are revisited in this section, but now making use of a fully quantum-mechanical treatment. I focus first on the single-detector case; the generalization to multiple detectors is straightforward and is given at the end of this section. As before, the photocurrent operator is given by Equation (9) and has Fourier components given by Equation (15). The shot noise intensity is measured in the same way: a filter $W(\nu)$ is applied to the photocurrent before a square-law detector and integrator are used to measure the intensity, as illustrated in Figure 7. This measurement scheme produces the quantity $P_{W, T}$ as defined by Equation (61). However, calculation of the statistics of $P_{W, T}$ now requires quantum operator averages, which I perform in the usual way appropriate for thermal radiation, namely by combining photon creation and destruction operators pairwise. The quantum computation of the second-order moment is detailed in Equation (19), with a result that is identical to the semiclassical second-order moment,

$$
\left\langle I\left(\nu_{1}\right) I^{\dagger}\left(\nu_{2}\right)\right\rangle=\left[\bar{\Gamma}^{2} \delta\left(\nu_{1}\right)+\bar{\Gamma}+S_{\Gamma}\left(\nu_{1}\right)\right] \delta\left(\nu_{1}-\nu_{2}\right) .
$$

In fact the semiclassical spectrum was chosen to coincide with the quantum result through the definitions of $\bar{\Gamma}$ and $S_{\Gamma}(\nu)$ given in Equations (57) and (58). Thus, we conclude that 
$\left\langle P_{W, T}\right\rangle=\bar{\Gamma} B T$ for the quantum calculation just as for the semiclassical case (Equation (61)), provided that we choose the noise filter $W(\nu)$ to avoid the excess low-frequency noise as discussed in Section 5.

Evaluation of the fluctuations of $P_{W, T}$ requires a quantum computation of the fourth-order moment of Fourier components of the photocurrent, which in turn requires eighth-order moments of the photon operators:

$$
\begin{aligned}
F\left(\nu_{1}, \nu_{2}, \nu_{3}, \nu_{4}\right)= & \left\langle I\left(\nu_{1}\right) I^{\dagger}\left(\nu_{2}\right) I\left(\nu_{3}\right) I^{\dagger}\left(\nu_{4}\right)\right\rangle \\
= & \int d \nu_{1}^{\prime} d \nu_{2}^{\prime} d \nu_{3}^{\prime} d \nu_{4}^{\prime}\left\langle b^{\dagger}\left(\nu_{1}^{\prime}\right) b\left(\nu_{1}^{\prime}+\nu_{1}\right)\right. \\
& \times b^{\dagger}\left(\nu_{2}^{\prime}+\nu_{2}\right) b\left(\nu_{2}^{\prime}\right) b^{\dagger}\left(\nu_{3}^{\prime}\right) \\
& \left.\times b\left(\nu_{3}^{\prime}+\nu_{3}\right) b^{\dagger}\left(\nu_{4}^{\prime}+\nu_{4}\right) b\left(\nu_{4}^{\prime}\right)\right\rangle .
\end{aligned}
$$

Combining operators pairwise produces $4 !=24$ terms. However, as for the semiclassical calculation, many of these represent DC terms that are rejected by the filter $W(\nu)$ and therefore do not contribute to the shot noise intensity. For example, if the first two operators are paired, we will have a factor

$$
\begin{aligned}
\left\langle 11^{\prime}\right\rangle & =\int d \nu_{1}^{\prime}\left\langle b^{\dagger}\left(\nu_{1}^{\prime}\right) b\left(\nu_{1}^{\prime}+\nu_{1}\right)\right\rangle \\
& =\int d \nu_{1}^{\prime} n\left(\nu_{1}^{\prime}\right) \delta\left(\nu_{1}\right)=\bar{\Gamma} \delta\left(\nu_{1}\right)
\end{aligned}
$$

which vanishes except at DC, $\nu_{1}=0$, and may therefore be ignored. This shows that we may ignore any similar pairing, e.g., $\left\langle 22^{\prime}\right\rangle$ in the obvious notation. Any pairing may be represented by a permutation, e.g., $\left\langle 11^{\prime}\right\rangle\left\langle 23^{\prime}\right\rangle\left\langle 34^{\prime}\right\rangle\left\langle 2^{\prime} 4\right\rangle$ corresponds to the permutation (1)(234) expressed in cyclic notation. All permutations that include a cycle of length 1, e.g., (3), will give DC terms that we may ignore. This leaves nine permutations left to consider:

$$
\begin{array}{lll}
(12)(34) & (1234) & (1243) \\
(13)(24) & (1324) & (1342) \\
(14)(23) & (1423) & (1432) .
\end{array}
$$

The detailed evaluation of some of these pairings is given in Appendix D. These pairings reproduce the terms found in the semiclassical calculation outlined in Section 5 and detailed in Appendix B, and also generate some extra terms that arise from the non-gaussianity of the photon arrival rate fluctuations as described in Appendix F that are neglected in our semiclassical calculation. In particular, the (1432) permutation includes the contributions expressed by Equations (D5) and (D6) in Appendix D:

$$
\left\langle P_{W, T}^{2}\right\rangle_{(1432)}=T\left[\bar{\Gamma}+S_{\Gamma}(0)\right]\left[\int d \nu|W(\nu)|^{2}\right]^{2}+\ldots
$$

which are same as the semiclassical terms D1 and and C1b listed in Appendix B that correspond to Poisson noise and bunching noise, respectively. The latter term contributes $S_{\Gamma}(0) / \bar{\Gamma}^{2}$ to the fractional fluctuations $\sigma_{P}^{2} /\left\langle P_{W, T}^{2}\right\rangle$ (see Equations (71), (78)) and thus represents the shot noise intensity fluctuations due to photon bunching. It is this term that gives the same $\sqrt{1+n}$ sensitivity degradation due to bunching as occurs for ordinary photon counting.

It is not difficult to translate these results to the case of multiple detectors. We again focus our attention on the (1432) operator pairing in the corresponding quantum calculation, which includes the contributions (Equations (D7) and (D8))

$$
\begin{aligned}
{\left[F_{a b c d}\right]_{(1432)}=} & T \delta_{a b} \delta_{c d}\left[\bar{\Gamma}_{a} \delta_{a c}+C_{a c}^{(\Gamma)}(0)\right] \\
& \times\left[\int d \nu|W(\nu)|^{2}\right]^{2}+\ldots
\end{aligned}
$$

that correspond to the Poisson and bunching terms D1 and C1b found in the semiclassical calculation, as outlined in Section 6 and detailed in Appendix C. The latter term contributes

$$
4 T C_{11}^{(\Gamma)}(0)\left[\int d \nu|W(\nu)|^{2}\right]^{2}
$$

to the measurement variance $\sigma_{P_{\Delta}}^{2}$ (Equation (93)) for the twodetector setup shown in Figures 1 and 6, and leads to the $\sqrt{1+n}$ photon bunching degradation.

Thus, we conclude that a full quantum calculation reproduces the conclusions of the semiclassical analyses for one or two detectors given in Sections 5 and 6, namely that shot noise intensity measurements cannot evade the bunching noise.

\section{CORRELATION OF SHOT NOISE AND PHOTON COUNTS}

We have two ways of measuring the photon flux: direct photon counting using the time integral of the photocurrent, $N_{T}$, defined in Equation (10), or through a shot noise intensity measurement represented by $P_{W, T}$ and defined in Equation (61). According to our semiclassical and quantum calculations, both are affected by photon bunching; therefore, these quantities must be correlated if our results are correct. Conversely, if we can establish a correlation between these quantities, the correlation may be used together with the well-known results for bunching noise in direct photon counting to establish a lower bound for the bunching noise that must also be present in the shot noise measurements. In this section, I present a fully quantum treatment of these topics.

We are interested in evaluating the correlation

$$
\begin{aligned}
\left\langle P_{W, T} N_{T}\right\rangle= & \int_{-\infty}^{+\infty} d \nu_{1} \int_{-\infty}^{+\infty} d \nu_{2} \\
& \times \int_{-\infty}^{+\infty} d \nu_{3}\left\langle I\left(\nu_{1}\right) I^{\dagger}\left(\nu_{2}\right) I\left(\nu_{3}\right)\right\rangle W\left(\nu_{1}\right) W^{*}\left(\nu_{2}\right) \\
& \times \int_{-T / 2}^{T / 2} d t_{1} e^{i 2 \pi\left(\nu_{1}-\nu_{2}\right) t_{1}} \int_{-T / 2}^{T / 2} d t_{3} e^{i 2 \pi \nu_{3} t_{3}} .
\end{aligned}
$$

We thus require the sixth-order moments of photon operators,

$$
\begin{gathered}
\left\langle I\left(\nu_{1}\right) I^{\dagger}\left(\nu_{2}\right) I\left(\nu_{3}\right)\right\rangle=\int d \nu_{1}^{\prime} d \nu_{2}^{\prime} d \nu_{3}^{\prime}\left\langle b^{\dagger}\left(\nu_{1}^{\prime}\right) b\left(\nu_{1}^{\prime}+\nu_{1}\right)\right. \\
\left.\times b^{\dagger}\left(\nu_{2}^{\prime}+\nu_{2}\right) b\left(\nu_{2}^{\prime}\right) b^{\dagger}\left(\nu_{3}^{\prime}\right) b\left(\nu_{3}^{\prime}+\nu_{3}\right)\right\rangle
\end{gathered}
$$

to be evaluated as usual by computing the $3 !=6$ operator pairings. As in Section 7, the noise filters $W\left(\nu_{1}\right)$ and $W^{*}\left(\nu_{2}\right)$ allow us to ignore the DC terms in those variables; however, we must now retain DC terms for $\nu_{3}$. We can thus neglect permutations involving the cycles (1) and (2), which leaves 
only (12)(3), (123), and (132). We find:

$$
\begin{aligned}
(12)(3)= & {\left[\bar{\Gamma}+S_{\Gamma}\left(\nu_{1}\right)\right] \bar{\Gamma} \delta\left(\nu_{1}-\nu_{2}\right) \delta\left(\nu_{3}\right) } \\
(123)= & \int d \nu_{1}^{\prime} n\left(\nu_{1}^{\prime}\right) n\left(\nu_{1}+\nu_{3}+\nu_{1}^{\prime}\right) \\
& \times\left[1+n\left(\nu_{1}+\nu_{1}^{\prime}\right)\right] \delta\left(\nu_{1}-\nu_{2}+\nu_{3}\right) \\
(132)= & \int d \nu_{1}^{\prime} n\left(\nu_{1}^{\prime}\right)\left[1+n\left(\nu_{1}+\nu_{1}^{\prime}\right)\right] \\
& \times\left[1+n\left(\nu_{1}+\nu_{1}^{\prime}-\nu_{2}\right)\right] \delta\left(\nu_{1}-\nu_{2}+\nu_{3}\right) .
\end{aligned}
$$

Performing the integrations indicated in Equation (104) gives

$$
\begin{aligned}
(12)(3)= & T^{2} \int_{-\infty}^{+\infty} d \nu_{1}\left|W\left(\nu_{1}\right)\right|^{2} \bar{\Gamma}\left[\bar{\Gamma}+S_{\Gamma}\left(\nu_{1}\right)\right] \\
(123)= & T \int_{-\infty}^{+\infty} d \nu_{1}\left|W\left(\nu_{1}\right)\right|^{2}\left[S_{\Gamma}\left(\nu_{1}\right)\right. \\
& \left.+\int d \nu_{1}^{\prime} n\left(\nu_{1}^{\prime}\right) n^{2}\left(\nu_{1}^{\prime}+\nu_{1}\right)\right] \\
(132)= & T \int_{-\infty}^{+\infty} d \nu_{1}\left|W\left(\nu_{1}\right)\right|^{2}\left[\bar{\Gamma}+S_{\Gamma}\left(\nu_{1}\right)\right. \\
& \left.+S_{\Gamma}(0)+\int d \nu_{1}^{\prime} n^{2}\left(\nu_{1}^{\prime}\right) n\left(\nu_{1}^{\prime}+\nu_{1}\right)\right] .
\end{aligned}
$$

The (12)(3) term just gives the product of averages $\left\langle P_{W, T}\right\rangle\left\langle N_{T}\right\rangle$, so

$$
\begin{aligned}
& \left\langle P_{W, T} N_{T}\right\rangle-\left\langle P_{W, T}\right\rangle\left\langle N_{T}\right\rangle=T \int_{-\infty}^{+\infty} d \nu_{1}\left|W\left(\nu_{1}\right)\right|^{2} \\
& \quad \times\left\{\bar{\Gamma}+S_{\Gamma}(0)+2 S_{\Gamma}\left(\nu_{1}\right)+\int d \nu_{1}^{\prime} n\left(\nu_{1}^{\prime}\right)\left[n\left(\nu_{1}^{\prime}\right)\right.\right. \\
& \left.\left.\quad+n\left(\nu_{1}^{\prime}+\nu_{1}\right)\right] n\left(\nu_{1}^{\prime}+\nu_{1}\right)\right\} .
\end{aligned}
$$

Most of the terms vanish if we design the noise filter to reject the low-frequency noise as illustrated by the hatched region in Figure 3; the terms that survive are

$$
\left\langle P_{W, T} N_{T}\right\rangle-\left\langle P_{W, T}\right\rangle\left\langle N_{T}\right\rangle=2 B T\left[\bar{\Gamma}+S_{\Gamma}(0)\right],
$$

using our standard definition of the shot noise measurement bandwidth $B$ (Equation (65)). We therefore see that the shot noise intensity $P_{W, T}$ and photon counts $N_{T}$ are indeed correlated, as we expect if both are affected by photon bunching.

The value of the correlation given by Equation (105) allows us to set a lower bound on the variance of the shot noise intensity $P_{W, T}$ given the well-established results for the variance of the photon counts $N_{T}$. Indeed, if $X$ and $Y$ are two random variables with zero mean and finite variance, the Cauchy-Schwarz inequality holds:

$$
\langle X Y\rangle^{2} \leqslant\left\langle X^{2}\right\rangle\left\langle Y^{2}\right\rangle,
$$

which establishes a lower limit for the variance of $X$,

$$
\left\langle X^{2}\right\rangle \geqslant \frac{\langle X Y\rangle^{2}}{\left\langle Y^{2}\right\rangle} \text {. }
$$

Now set $X=P_{W, T}-\left\langle P_{W, T}\right\rangle$ and $Y=N_{T}-\left\langle N_{T}\right\rangle$. From Equations (12), (57), and (58) we have

$$
\left\langle Y^{2}\right\rangle=T\left[\bar{\Gamma}+S_{\Gamma}(0)\right] .
$$

Using the known value of the correlation $\langle X Y\rangle$ given by Equation (105), we have

$$
\sigma_{P}^{2}=\left\langle X^{2}\right\rangle \geqslant \frac{\langle X Y\rangle^{2}}{\left\langle Y^{2}\right\rangle}=4 B^{2} T\left[\bar{\Gamma}+S_{\Gamma}(0)\right] .
$$

Dividing by the square of the mean value $\left\langle P_{W, T}\right\rangle=2 B T \bar{\Gamma}$ gives the fractional fluctuations

$$
\frac{\sigma_{P}^{2}}{\left\langle P_{W, T}\right\rangle^{2}} \geqslant \frac{1}{\bar{\Gamma} T}+\frac{S_{\Gamma}(0)}{\bar{\Gamma}^{2} T}=\frac{1+n}{\bar{\Gamma} T}
$$

where we have used Equations (76) and (77) in writing the second expression. Thus, using a fully quantum-mechanical calculation, we have demonstrated that the shot noise intensity measurement must suffer at least the same $\sqrt{1+n}$ sensitivity degradation due to photon bunching as does standard photon counting. Comparison to the result of the semiclassical calculation, Equation (78), shows that the correlation bound does not include the $1 / B T$ noise term associated with a finite bandwidth for the shot noise measurement. This is to be expected: the finite-bandwidth noise does not influence the direct photon counts, represented by $N_{T}$, and is therefore absent in the correlation $\left\langle P_{W, T} N_{T}\right\rangle$.

The extension to the case of two detectors is straightforward but will be omitted. However, it is easy to see that the results above may be applied independently to each detector in the two-detector setup shown in Figure 1. Thus, the shot noise intensity for each detector must be correlated with its photocurrent. Furthermore, the two photocurrents are correlated, as demonstrated by Hanbury Brown \& Twiss (1956). Therefore, the (high-frequency) shot noise intensities of the two detectors must also be correlated, even though the shot noise itself is not: this distinction, between moments of the form $\left\langle I_{1}^{2} I_{2}^{2}\right\rangle$ versus $\left\langle I_{1} I_{2}\right\rangle$, is elucidated further in Section 10.

\section{COMPARISON TO THE RESULTS OF LIEU ET AL.}

In this section, I compare the results of the previous sections with those of Lieu et al. (2015) for both of the regimes they examine, corresponding to long sample times $\Delta \nu \Delta t \gg 1$ and short sample times $\bar{\Gamma} \Delta t<1$. Here $\Delta t$ is the single-sample time defined by Lieu et al. (2015); to avoid confusion, I use $\Delta t$ instead of their chosen symbol, $T$, and instead reserve $T=N \Delta t$ to signify the total time duration of the measurement required for the acquisition and integration of $N$ samples. The concept of sample time does not arise in my calculations since I assume continuous time integration; however, a connection can readily be made since the sample time $\Delta t$ defined by Lieu et al. sets the shot noise bandwidth $B_{\Delta t}=1 / 2 \Delta t$ associated with their measurement scheme. I make use of this correspondence to compare the two calculations for the same total measurement duration $T$, and find that the results agree in the long sample time regime but disagree for short sample times. Thus, my results directly contradict the claim of Lieu et al. that bunching noise may be avoided in the latter limit. It is important to note that for both regimes, the total measurement duration $T$ can be chosen to satisfy $\Delta \nu T \gg 1$, as I have assumed for my calculations; indeed, long measurement durations are essential for astronomical observations since the sensitivity improves as $1 / \sqrt{T}$. To aid in comparison of the results, and for ease of reference in the discussion below, the relevant quantities and 
Table 1

Comparison of Symbols in this Paper vs. those of Lieu et al. (2015)

\begin{tabular}{|c|c|c|c|}
\hline Quantity & This Paper & $\begin{array}{c}\text { Lieu et al. } \\
\text { (As Used Here) }\end{array}$ & $\begin{array}{c}\text { Lieu et al. } \\
\text { (Original } \\
\text { Notation) }\end{array}$ \\
\hline Number of samples & (continuous) & $N$ & $N$ \\
\hline Sample time & (continuous) & $\Delta t$ & $T$ \\
\hline $\begin{array}{c}\text { Total measure- } \\
\text { ment time }\end{array}$ & $T$ & $T=N \Delta t$ & $N T$ \\
\hline Optical bandwidth & $\Delta \nu$ & $\Delta \nu=1 / \tau$ & $1 / \tau$ \\
\hline Shot noise bandwidth & $B$ & $B_{\Delta t}=1 / 2 \Delta t$ & $1 / 2 T$ \\
\hline Photon arrival rate & $\bar{\Gamma}=n \Delta \nu$ & $n_{0} \Delta \nu$ & $n_{0} / \tau$ \\
\hline $\begin{array}{l}\text { Photon rate } \\
\text { fluctuations }\end{array}$ & $S_{\Gamma}(0)$ & $\sqrt{\pi} n_{0}^{2} \Delta \nu$ & $\sqrt{\pi} n_{0}^{2} / \tau$ \\
\hline
\end{tabular}

symbols used to represent them in both papers are provided in Table 1.

Lieu et al. (2015) present a fully quantum calculation of the shot noise fluctuations for the two-detector setup illustrated in Figure 1. They use a very similar quantum formalism for photon detection that differs only in minor and inconsequential detail. For example, their definition of the operator representing the detector output measures photon power instead of photon counts as can be seen from their Equation (5). Moments of photon operators are calculated in the standard way, by combining operators pairwise, as is appropriate for thermal radiation. Lieu et al. consider the detector output averaged over a sample time $\Delta t$ corresponding to the quantity

$$
I_{\Delta t}=\frac{1}{\Delta t} \int_{0}^{\Delta t} d t I(t)
$$

in my notation. Lieu et al. take the difference of the outputs of the two detectors in Figure 1,

$$
I_{\Delta}(t)=I_{1}(t)-I_{2}(t),
$$

and calculate both the second and fourth moments of

$$
I_{\Delta, \Delta t}=\frac{1}{\Delta t} \int_{0}^{\Delta t} d t I_{\Delta}(t)
$$

Their fundamental conclusions rely on evaluation of the mean and variance of the sum of $N$ consecutive measurements of $\left[I_{\Delta, \Delta t}\right]^{2}$, obtained over a total time duration of $T=N \Delta t$. This quantity may be expressed as

$$
P_{N, \mathrm{LKD}}=\sum_{k=1}^{N}\left[I_{\Delta, \Delta t}(k)\right]^{2}
$$

where

$$
I_{\Delta, \Delta t}(k)=\frac{1}{\Delta t} \int_{(k-1) \Delta t}^{k \Delta t} d t I_{\Delta}(t)
$$

are the consecutive time-averaged samples of the photocurrent difference $I_{\Delta}(t)$. In contrast, I first apply an arbitrary linear filter to the photocurrent,

$$
I_{\Delta}^{(W)}(t)=\int_{-\infty}^{t} d t^{\prime} W\left(t-t^{\prime}\right) I_{\Delta}\left(t^{\prime}\right)
$$

and then study the mean and variance of the shot noise intensity integrated over time,

$$
P_{\Delta}=\int_{-T / 2}^{T / 2} d t\left[I_{\Delta}^{(W)}(t)\right]^{2}
$$

as illustrated in Figure 8.

Although the definitions of $P_{N, \mathrm{LKD}}$ and $P_{\Delta}$ superficially appear to be different, these two quantities are closely related, as illustrated in Figure 9. The averaging over $\Delta t$ performed by Lieu et al. (2015) may be represented by a particular (and inflexible) choice for the linear filter, namely a time window function:

$$
W_{\Delta t}(t)= \begin{cases}1 / \Delta t, & 0 \leqslant t \leqslant \Delta t \\ 0, & \text { otherwise. }\end{cases}
$$

According to our definition (Equation (65)), this filter has a bandwidth

$$
2 B_{\Delta t}=\int_{-\infty}^{+\infty} d \nu\left|W_{\Delta t}(\nu)\right|^{2}=\int_{-\infty}^{+\infty} d t W_{\Delta t}^{2}(t)=\frac{1}{\Delta t}
$$

we will also need

$$
\int_{-\infty}^{+\infty} d \nu\left|W_{\Delta t}(\nu)\right|^{4}=\frac{2}{3 \Delta t}
$$

Note that this filter does not reject DC or low-frequency noise, but these are automatically rejected anyway by differencing the currents in a two-detector setup. Another distinction is that Lieu et al. (2015) perform the time integration operation as a discrete sum rather than a continuous integration: the output of the square-law detector is sampled at times $t_{k}=k \Delta t$, and then summed, as represented by the dashed box in Figure 9. This choice does not significantly affect the results, though the discrete sampling operation of Lieu et al. may result in a minor degradation in performance due to noise aliasing.

Equation (29) in Lieu et al. (2015) gives the second moment of one sample:

$$
\left\langle I_{\Delta, \Delta t}^{2}\right\rangle=\frac{n_{0}}{\Delta t \tau}
$$

where we have omitted their factor of $\omega_{0}^{2}$ so that the operator represents photon flux. According to their Equation (7), their symbol $\tau$ is related to the optical coherence time and is inversely proportional to the optical bandwidth, $\tau \sim 1 / \Delta \nu$. Thus, translated to our notation,

$$
\left\langle I_{\Delta, \Delta t}^{2}\right\rangle \sim n_{0} \Delta \nu 2 B_{\Delta t}=2 \bar{\Gamma} B_{\Delta t}
$$

where $\bar{\Gamma}=n_{0} \Delta \nu$ is the photon rate before the beamsplitter. Meanwhile, the corresponding equation for our observable (Equation (91)) reads

$$
\left\langle P_{\Delta}\right\rangle=2 B T \bar{\Gamma} .
$$

These may be reconciled by using Equation (117),

$$
\left\langle\left[I_{\Delta}^{(W)}(t)\right]^{2}\right\rangle=\lim _{T \rightarrow 0} \frac{\left\langle P_{\Delta}\right\rangle}{T}=2 \bar{\Gamma} B
$$




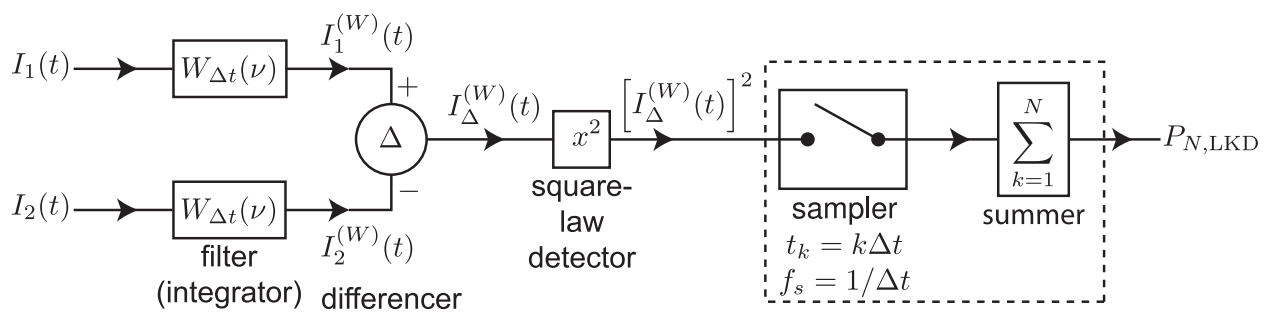

Figure 9. Signal flow diagram for shot-noise detection as proposed by Lieu et al. (2015). The ordering of the filtering and differencing operations may be interchanged. This scheme differs from that shown in Figure 8 in two ways: (1) the choice of filter is fixed and corresponds to boxcar integrator with time duration $\Delta t$; and (2) the (slow) time integration is not continuous but is instead performed in a discrete fashion using a sampler and summer (dashed box). The sampler operates at a rate $1 / \Delta t$ and is synchronized to the filters.

Thus, our results for the mean value of the shot noise intensity agree with Lieu et al. (2015) if we make the replacements $\tau \rightarrow 1 / \Delta \nu$ and $B \rightarrow B_{\Delta t}=1 / 2 \Delta t$.

We now turn to the variance of a single output sample of the Lieu et al. (2015) setup, which they calculate using a clever evaluation of the eighth-order moments of the photon operators in which most of the terms are discarded since they cancel in the two-detector scheme. Specifically, they calculate

$$
\sigma_{1, \mathrm{LKD}}^{2}=\left\langle I_{\Delta, \Delta t}^{4}\right\rangle-\left\langle I_{\Delta, \Delta t}^{2}\right\rangle^{2}
$$

and express the result as a fractional variance in their Equation (32) when the number of samples $N=1$,

$$
\frac{\sigma_{1, \mathrm{LKD}}^{2}}{\left\langle I_{\Delta, \Delta t}^{2}\right\rangle^{2}}=2+3 \frac{\tau}{\Delta t} F\left(\frac{\Delta t}{\tau}\right)+\frac{\tau}{\Delta t n_{0}} .
$$

Translated into our notation, this reads

$$
\frac{\sigma_{1, \mathrm{LKD}}^{2}}{\left\langle I_{\Delta, \Delta t}^{2}\right\rangle^{2}}=2+\frac{3}{\Delta \nu \Delta t} F(\Delta \nu \Delta t)+\frac{1}{\bar{\Gamma} \Delta t} .
$$

Here $F(x)$ is a smooth function that allows both the $\Delta \nu \Delta t \ll 1$ and $\Delta \nu \Delta t \gg 1$ limits to be examined, and is derived under the assumption of a Gaussian spectral profile for the thermal radiation. Comparison of the single-sample variance with our results requires use of the latter limit because we assume $\Delta \nu T \gg 1$, where $T$ is the duration of the measurement, for evaluation of the Fourier integrals. In this limit, $F(x) \rightarrow \sqrt{\pi}$, and

$$
\frac{\sigma_{1, \mathrm{LKD}}^{2}}{\left\langle I_{\Delta, \Delta t}^{2}\right\rangle^{2}}=2+\frac{3 \sqrt{\pi}}{\Delta \nu \Delta t}+\frac{1}{\bar{\Gamma} \Delta t} .
$$

We may safely assume that consecutive samples are uncorrelated in the $\Delta \nu \Delta t \gg 1$ limit, because the sample time $\Delta t$ is long compared to the optical coherence time $\tau$. Thus, the fractional variance for a sum of $N$ samples (Equation (114)) would be

$$
\frac{\sigma_{N, \mathrm{LKD}}^{2}}{\left\langle I_{\Delta, \Delta t}^{2}\right\rangle^{2}}=\frac{1}{N}\left[2+\frac{3 \sqrt{\pi}}{\Delta \nu \Delta t}+\frac{1}{\bar{\Gamma} \Delta t}\right] .
$$

Meanwhile, our result for the fractional variance (Equation (96)) reads

$$
\begin{aligned}
\frac{\sigma_{P_{\Delta}}^{2}}{\left\langle P_{\Delta}\right\rangle^{2}}= & \frac{1}{T}\left[\frac{2 \int d \nu|W(\nu)|^{4}}{\left[\int d \nu|W(\nu)|^{2}\right]^{2}}+\frac{S_{\Gamma}(0)}{\bar{\Gamma}^{2}}\right. \\
& +\frac{2 \int d \nu d \nu^{\prime}|W(\nu)|^{2}\left|W\left(\nu^{\prime}\right)\right|^{2} S_{\Gamma}\left(\nu-\nu^{\prime}\right)}{\bar{\Gamma}^{2}\left[\int d \nu|W(\nu)|^{2}\right]^{2}} \\
& \left.+\frac{1}{\bar{\Gamma}}\right] .
\end{aligned}
$$

The first term is readily evaluated using Equations (118) and (119). The numerator in the third term must be evaluated in our chosen limit $\Delta \nu \Delta t \gg 1$, so $|\nu|,\left|\nu^{\prime}\right| \ll \Delta \nu$ for the integrals; we obtain

$$
\frac{\sigma_{P_{\Delta}}^{2}}{\left\langle P_{\Delta}\right\rangle^{2}}=\frac{1}{T}\left[\frac{4}{3} \Delta t+3 \frac{S_{\Gamma}(0)}{\bar{\Gamma}^{2}}+\frac{1}{\bar{\Gamma}}\right] .
$$

For the Gaussian spectral profile used by Lieu et al. (2015),

$$
\begin{gathered}
\bar{\Gamma}=\int_{-\infty}^{+\infty} d \nu n(\nu)=\frac{n_{0}}{\tau} \rightarrow n_{0} \Delta \nu \\
S_{\Gamma}(0)=\int_{-\infty}^{+\infty} d \nu n^{2}(\nu)=\frac{\sqrt{\pi} n_{0}^{2}}{\tau} \rightarrow \sqrt{\pi} n_{0}^{2} \Delta \nu,
\end{gathered}
$$

so our result reads

$$
\frac{\sigma_{P}^{2}}{\left\langle P_{\Delta}\right\rangle^{2}}=\frac{4}{3} \frac{\Delta t}{T}+\frac{3 \sqrt{\pi}}{\Delta \nu T}+\frac{1}{\bar{\Gamma} T}
$$

Correspondence with Lieu et al. (2015) is obtained by letting the total measurement time $T$ coincide with $N \Delta t$, the time to obtain $N$ samples, yielding

$$
\frac{\sigma_{P}^{2}}{\left\langle P_{\Delta}\right\rangle^{2}}=\frac{1}{N}\left[\frac{4}{3}+\frac{3 \sqrt{\pi}}{\Delta \nu \Delta t}+\frac{1}{\bar{\Gamma} \Delta t}\right] .
$$

This expression reproduces the three terms of the Lieu et al. result stated in Equation (128), which is derived from their Equation (32), apart from a somewhat smaller numerical factor on our first term which likely results from our use of a continuous integration over the measurement time $T$ instead of a sum of discrete samples taken every $\Delta t$ as illustrated in Figure 9. Note that the second term of the Lieu et al. result confirms our $S_{\Gamma}(0)$ term, which is the signature of photon 
bunching in the shot noise intensity. Thus, our results agree in the long sample time limit, $\Delta \nu \Delta t \gg 1$.

In contrast, there is a major disagreement in the short sample time regime, $\bar{\Gamma} \Delta t<1$, which corresponds to the use of a wide bandwidth for measurement of the shot noise, $B_{\Delta t}>\bar{\Gamma}=n_{0} \Delta \nu \gg \Delta \nu$ as illustrated in Figures 3 and 4 . Lieu et al. find $F(x) \approx 1 / x$ for $x \ll 1$, so their result (Equation (126)) in this limit becomes

$$
\frac{\sigma_{1, \mathrm{LKD}}^{2}}{\left\langle I_{\Delta, \Delta t}^{2}\right\rangle^{2}} \approx 5+\frac{1}{\bar{\Gamma} \Delta t} \approx \frac{1}{\bar{\Gamma} \Delta t} .
$$

In other words, the goal is to choose $\Delta t$ small enough that the Poisson term dominates the single-sample variance. Their principal claim, namely that bunching noise can be avoided, rests on the statement that a sum of $N$ such samples, as given by Equation (114) and illustrated in Figure 9, and acquired over a total measurement time $T=N \Delta t$, would have a fractional variance

$$
\frac{\sigma_{N, \mathrm{LKD}}^{2}}{\left\langle P_{N, \mathrm{LKD}}\right\rangle}=\frac{1}{N} \frac{\sigma_{1, \mathrm{LKD}}^{2}}{\left\langle I_{\Delta, \Delta t}^{2}\right\rangle^{2}}=\frac{1}{N \bar{\Gamma} \Delta t}=\frac{1}{\bar{\Gamma} T},
$$

as would be expected if the samples were statistically independent. Note that the limit $\Delta \nu T \gg 1$ as required for our calculation of the same two-detector scheme is reached simply by choosing $N \gg 1 / \Delta \nu \Delta t$, so the comparison is immediate through use of Equations (96), (71), and (78):

$$
\frac{\sigma_{P_{\Delta}}^{2}}{\left\langle P_{\Delta}^{2}\right\rangle}=\frac{1}{B T}+\frac{1+n}{\bar{\Gamma} T} \text {. }
$$

The second term dominates because we have assumed $B>\bar{\Gamma}$. Thus, our calculation gives a sensitivity of $(1+n) / \bar{\Gamma} T$ while Lieu et al. find $1 / \bar{\Gamma} T$; our result includes the $(1+n)$ bunching penalty, while Lieu et al. claim it can be avoided.

\section{RESOLVING THE CONTRADICTION}

Section 9 shows that the detection sensitivities derived in this paper agree with those of Lieu et al. (2015) for long sample times but disagree in the short sample time regime that is of central interest. In obtaining their result for short sample times (Equation (135)), Lieu et al. assume statistical independence but do not actually prove this by computing the correlations between samples, $\left\langle\left[I_{\Delta, \Delta t}(k)\right]^{2}\left[I_{\Delta, \Delta t}(l)\right]^{2}\right\rangle$. They instead state, after their Equation (32): "...data in non-overlapping time periods are uncorrelated, because the correlation function $\left\langle I_{T}^{d}(t) I_{T}^{d}(0)\right\rangle$ is proportional to a delta function... ." Translated to our notation, their statement relates to

$$
\begin{aligned}
\left\langle I_{\Delta, \Delta t}(k) I_{\Delta, \Delta t}(l)\right\rangle= & \frac{1}{(\Delta t)^{2}} \int_{(k-1) \Delta t}^{k \Delta t} d t_{1} \\
& \times \int_{(l-1) \Delta t}^{l \Delta t} d t_{2}\left\langle I_{\Delta}\left(t_{1}\right) I_{\Delta}\left(t_{2}\right)\right\rangle \\
= & \delta_{k l} \frac{\bar{\Gamma}}{\Delta t} .
\end{aligned}
$$

This demonstrates that $I_{\Delta, \Delta t}(k)$ are uncorrelated, as Lieu et al. claim, which is to be expected because the spectral density of
$I_{\Delta}(t)$ is white as illustrated in Figure 4. Nonetheless, this does not mean that the squares of these random variables, $\left[I_{\Delta, \Delta t}(k)\right]^{2}$, are uncorrelated.

Thus, we see that the fundamental claim of Lieu et al. is invalidated by a simple error, the fallacy of the converse. Suppose we have two zero-mean random variables, $X$ and $Y$. If they are independent, they must be uncorrelated, because $\langle X Y\rangle=\langle X\rangle\langle Y\rangle=0$. However, the converse is not necessarily true. If it were true, we could claim that when $\langle X Y\rangle=0$, we must also have $\left\langle X^{2} Y^{2}\right\rangle=\left\langle X^{2}\right\rangle\left\langle Y^{2}\right\rangle$, which is the statement upon which the Lieu et al. result rests. A simple counterexample suffices: suppose the joint distribution of $X$ and $Y$ is given by

$$
f(x, y)=\frac{1}{2}[\delta(x-y)+\delta(x+y)] g(x),
$$

where $g(x)$ is a Gaussian distribution with zero mean and variance $\sigma^{2}$. This distribution cannot be factorized into the form $f(x, y)=f_{x}(x) f_{y}(y)$, so clearly $X$ and $Y$ cannot be independent. We may readily compute

$$
\begin{aligned}
\langle X\rangle & =\int d x d y f(x, y) x=\int d x g(x) x=0 \\
\langle Y\rangle & =\int d x d y f(x, y) y=\int d x \frac{1}{2}[x-x] g(x)=0 \\
\langle X Y\rangle & =\int d x d y f(x, y) x y=\frac{1}{2} \int d x\left[x^{2}-x^{2}\right] g(x)=0 \\
\left\langle X^{2}\right\rangle & =\int d x d y f(x, y) x^{2}=\int d x g(x) x^{2}=\sigma^{2} \\
\left\langle Y^{2}\right\rangle & =\int d x d y f(x, y) y^{2}=\frac{1}{2} \int d x\left[x^{2}+x^{2}\right] g(x)=\sigma^{2} \\
\left\langle X^{2} Y^{2}\right\rangle & =\int d x d y f(x, y) x^{2} y^{2}=\frac{1}{2} \int d x\left[x^{4}+x^{4}\right] g(x)=3 \sigma^{4} .
\end{aligned}
$$

Thus $\left\langle X^{2} Y^{2}\right\rangle \neq\left\langle X^{2}\right\rangle\left\langle Y^{2}\right\rangle$ even though $\langle X Y\rangle=0$.

It is quite easy to see that the samples $I_{\Delta, \Delta t}^{2}(k)$ must be correlated using a simple physical argument. Consider the quantity defined in (Equation (115)):

$$
I_{\Delta, \Delta t}(k)=\frac{1}{\Delta t} \int_{(k-1) \Delta t}^{k \Delta t} d t\left[I_{1}(t)-I_{2}(t)\right] .
$$

There are only three events that can occur with non-negligible probability when $\bar{\Gamma} \Delta t \ll 1$, corresponding to a short sample time: (a) detector 1 receives a photon; (b) detector 2 receives a photon; (c) neither detector receives a photon. These events correspond to values of $I_{\Delta, \Delta t}(k)=\{+1 / \Delta t,-1 / \Delta t, 0\}$, respectively; therefore, $I_{\Delta, \Delta t}^{2}(k)$ takes on the value of $1 /(\Delta t)^{2}$ if either detector receives a photon, and zero otherwise. Thus, in the limit $\bar{\Gamma} \Delta t \ll 1$, the measurement scheme proposed by Lieu et al. (2015) (Equation (114)) can be expressed as

$$
P_{N, \mathrm{LKD}}=\sum_{k=1}^{N}\left[I_{\Delta, \Delta t}(k)\right]^{2}=\frac{N_{T}}{(\Delta t)^{2}}
$$

where $N_{T}$ is the total number of photons received by both detectors over the course of a measurement of duration $T=N \Delta t$. Note that a single detector, replacing the two detectors and beamsplitter, would also have received $N_{T}$ photons during such a measurement, so the statistics of the Lieu et al. observable $P_{N, \text { LKD }}$ must be the same as those for $N_{T}$, 
corresponding to photon counting with a single detector. The statistics of the latter are well known to be affected by bunching, as stated in Equation (13); thus the Lieu et al. claim that the bunching noise can be avoided is contradicted not only by the calculations presented in this paper, but also by the extensive experimental and theoretical work on photon bunching over the past six decades. A more rigorous discussion is given in Appendix E, which provides a detailed quantummechanical calculation that demonstrates that the samples $I_{\Delta, \Delta t}^{2}(k)$ are indeed correlated, and that accounting for these correlations in the sensitivity calculation leads again to the standard photon bunching penalty, in agreement with the calculations for both one and two detectors presented in Sections 5-8.

I thank Jim Moran and John Kovac at Harvard for bringing this interesting problem to my attention, and Richard Lieu, Tom Kibble, and Lingze Duan for extensive discussions. This paper is dedicated to the memory of my father, Jonas Stasys Zmuidzinas, who first introduced me to coherent-state integrals.

\section{APPENDIX A \\ GENERATING FUNCTION FOR A POISSON PROCESS WITH A TIME-VARIABLE RATE}

Here we evaluate the generating function introduced in Equation (45),

$$
G_{N}(s)=\left\langle e^{s N_{T}}\right\rangle=\sum_{k=0}^{\infty} \frac{s^{k}}{k !} \sum_{i_{1} \ldots i_{k}=1}^{M}\left\langle y_{i_{1}} \ldots y_{i_{k}}\right\rangle .
$$

If the indices $\left\{i_{1} \ldots i_{k}\right\}$ are all distinct, we may write

$$
\left\langle y_{i_{1}} \ldots y_{i_{k}}\right\rangle=\left\langle y_{i_{1}}\right\rangle \ldots\left\langle y_{i_{k}}\right\rangle=\Gamma\left(t_{i_{1}}\right) \ldots \Gamma\left(t_{i_{k}}\right) \Delta t_{i_{1}} \ldots \Delta t_{i_{k}},
$$

because the $y_{i}$ are independent. When one or more indices repeat, we may use $y_{i}^{m}=y_{i}$ (for $m \geqslant 1$ ) to again obtain a product of distinct factors. We are thus faced with the problem of partitioning the set of indices $\left\{i_{1} \ldots i_{k}\right\}$ into one or more groups, where the indices belonging to a group have the same value, and indices belonging to different groups have distinct values. The number of partitions of $k$ objects into $p$ groups is given by the Stirling number of the second kind, $S(k, p)$, which are nonzero for $p \leqslant k$ (Blasiak et al. 2007). We therefore write

$$
\sum_{i_{1} \ldots i_{k}=1}^{N} y_{i_{1}} \ldots y_{i_{k}}=\sum_{p=0}^{k} S(k, p) \sum_{i_{1} \ldots i_{p}}^{\prime} y_{i_{1}} \ldots y_{i_{p}}
$$

where the prime on the second sum indicates that the indices take on only distinct values. We may make the replacement

$$
\sum_{i_{1} \ldots i_{p}}^{\prime} y_{i_{1}} \ldots y_{i_{p}} \rightarrow p ! \sum_{i_{1}>i_{2} \ldots>i_{p}} y_{i_{1}} \ldots y_{i_{p}},
$$

by considering permutations of the indices. Taking the average,

$$
\begin{aligned}
\sum_{i_{1}>i_{2} \ldots>i_{p}}\left\langle y_{i_{1}} \ldots y_{i_{p}}\right\rangle= & \sum_{i_{1}>i_{2} \ldots>i_{p}} \Gamma\left(t_{i_{1}}\right) \ldots \Gamma\left(t_{i_{p}}\right) \Delta t_{i_{1}} \ldots \Delta t_{i_{p}} \\
\approx & \int_{0}^{T} d t_{1} \int_{0}^{t_{1}} d t_{2} \ldots \\
& \times \int_{0}^{t_{p-1}} d t_{p} \Gamma\left(t_{1}\right) \ldots \Gamma\left(t_{p}\right) \\
= & \frac{1}{p !}\left[\int_{0}^{T} d t \Gamma(t)\right]^{p} .
\end{aligned}
$$

Use of the following identity for the Stirling numbers

$$
\sum_{k, p=0}^{\infty} S(k, p) \frac{x^{k}}{k !} y^{p}=\exp \left[y\left(e^{x}-1\right)\right]
$$

allows us to evaluate the generating function,

$$
\begin{aligned}
G_{N}(s) & =\sum_{k, p=0}^{\infty} S(k, p) \frac{s^{k}}{k !}\left[\int_{0}^{T} d t \Gamma(t)\right]^{p} \\
& =\exp \left[\mu\left(e^{s}-1\right)\right],
\end{aligned}
$$

where

$$
\mu=\int_{0}^{T} d t \Gamma(t)
$$

\section{APPENDIX B \\ DETAILED EVALUATION OF SHOT NOISE FLUCTUATIONS: SINGLE DETECTOR}

The required average in Equation (69) may be performed by considering the partitions of the indices (see also Picinbono et al. 1970, Equation (2.16)):

$$
\begin{aligned}
\left\langle y_{i} y_{j} y_{k} y_{l}\right\rangle= & \left\langle y_{i}\right\rangle\left\langle y_{j}\right\rangle\left\langle y_{k}\right\rangle\left\langle y_{l}\right\rangle \\
& +\delta_{i j}\left\langle y_{i}\right\rangle\left\langle y_{k}\right\rangle\left\langle y_{l}\right\rangle+\delta_{i k}\left\langle y_{i}\right\rangle \\
& \times\left\langle y_{j}\right\rangle\left\langle y_{l}\right\rangle+\delta_{i l}\left\langle y_{i}\right\rangle\left\langle y_{j}\right\rangle\left\langle y_{k}\right\rangle \\
& +\delta_{j k}\left\langle y_{i}\right\rangle\left\langle y_{j}\right\rangle\left\langle y_{l}\right\rangle+\delta_{j l}\left\langle y_{i}\right\rangle \\
& \times\left\langle y_{j}\right\rangle\left\langle y_{k}\right\rangle+\delta_{k l}\left\langle y_{i}\right\rangle\left\langle y_{j}\right\rangle\left\langle y_{k}\right\rangle \\
& +\delta_{i j} \delta_{k l}\left\langle y_{i}\right\rangle\left\langle y_{k}\right\rangle+\delta_{i k} \delta_{j l}\left\langle y_{i}\right\rangle\left\langle y_{j}\right\rangle \\
& +\delta_{i l} \delta_{j k}\left\langle y_{i}\right\rangle\left\langle y_{j}\right\rangle \\
& +\delta_{i j} \delta_{i k}\left\langle y_{i}\right\rangle\left\langle y_{l}\right\rangle+\delta_{i j} \delta_{i l}\left\langle y_{i}\right\rangle\left\langle y_{k}\right\rangle \\
& +\delta_{j k} \delta_{j l}\left\langle y_{i}\right\rangle\left\langle y_{j}\right\rangle \\
& +\delta_{i k} \delta_{i l}\left\langle y_{i}\right\rangle\left\langle y_{j}\right\rangle+\delta_{i j} \delta_{i k} \delta_{i l}\left\langle y_{i}\right\rangle .
\end{aligned}
$$

We have neglected to subtract the correction terms such as $\left\langle y_{i}\right\rangle^{2}\left\langle y_{k}\right\rangle\left\langle y_{l}\right\rangle$ because, as in Section 4, they contain an extra factor of $\Delta t_{i}$ and therefore will vanish in the continuum limit. The number of terms of each partition class, here labeled A, B, $\mathrm{C}$, and $\mathrm{D}$, is $(1,6,7,1)$ and follows the sequence of Stirling numbers $S(4, k)$ (Blasiak et al. 2007), as expected. Taking the continuum limit and evaluating the Fourier integrals gives the 
following terms:

$$
\begin{aligned}
& \left\langle\hat{I}\left(\nu_{1}\right) \hat{I}^{*}\left(\nu_{2}\right) \hat{I}\left(\nu_{3}\right) \hat{I}^{*}\left(\nu_{4}\right)\right\rangle_{y} \\
& =\left[\bar{\Gamma} \delta\left(\nu_{1}\right)+\delta \hat{\Gamma}\left(\nu_{1}\right)\right]\left[\bar{\Gamma} \delta\left(\nu_{2}\right)\right. \\
& \left.+\delta \hat{\Gamma}^{*}\left(\nu_{2}\right)\right]\left[\bar{\Gamma} \delta\left(\nu_{3}\right)\right. \\
& \left.+\delta \hat{\Gamma}\left(\nu_{3}\right)\right]\left[\bar{\Gamma} \delta\left(\nu_{4}\right)+\delta \hat{\Gamma}^{*}\left(\nu_{4}\right)\right] \\
& +\left[\bar{\Gamma} \delta\left(\nu_{1}-\nu_{2}\right)+\delta \hat{\Gamma}\left(\nu_{1}-\nu_{2}\right)\right]\left[\bar{\Gamma} \delta\left(\nu_{3}\right)\right. \\
& \left.+\delta \hat{\Gamma}\left(\nu_{3}\right)\right]\left[\bar{\Gamma} \delta\left(\nu_{4}\right)+\delta \hat{\Gamma}^{*}\left(\nu_{4}\right)\right] \\
& +\left[\bar{\Gamma} \delta\left(\nu_{1}+\nu_{3}\right)+\delta \hat{\Gamma}\left(\nu_{1}+\nu_{3}\right)\right]\left[\bar{\Gamma} \delta\left(\nu_{2}\right)\right. \\
& \left.+\delta \hat{\Gamma}^{*}\left(\nu_{2}\right)\right]\left[\bar{\Gamma} \delta\left(\nu_{4}\right)+\delta \hat{\Gamma}^{*}\left(\nu_{4}\right)\right] \\
& +\left[\bar{\Gamma} \delta\left(\nu_{1}-\nu_{4}\right)+\delta \hat{\Gamma}\left(\nu_{1}-\nu_{4}\right)\right]\left[\bar{\Gamma} \delta\left(\nu_{2}\right)\right. \\
& \left.+\delta \hat{\Gamma}^{*}\left(\nu_{2}\right)\right]\left[\bar{\Gamma} \delta\left(\nu_{3}\right)+\delta \hat{\Gamma}\left(\nu_{3}\right)\right] \\
& +\left[\bar{\Gamma} \delta\left(\nu_{2}-\nu_{3}\right)+\delta \hat{\Gamma}\left(\nu_{2}-\nu_{3}\right)\right]\left[\bar{\Gamma} \delta\left(\nu_{1}\right)\right. \\
& \left.+\delta \hat{\Gamma}\left(\nu_{1}\right)\right]\left[\bar{\Gamma} \delta\left(\nu_{4}\right)+\delta \hat{\Gamma}^{*}\left(\nu_{4}\right)\right] \\
& +\left[\bar{\Gamma} \delta\left(\nu_{2}+\nu_{4}\right)+\delta \hat{\Gamma}\left(\nu_{2}+\nu_{4}\right)\right]\left[\bar{\Gamma} \delta\left(\nu_{1}\right)\right. \\
& \left.+\delta \hat{\Gamma}\left(\nu_{1}\right)\right]\left[\bar{\Gamma} \delta\left(\nu_{3}\right)+\delta \hat{\Gamma}\left(\nu_{3}\right)\right] \\
& +\left[\bar{\Gamma} \delta\left(\nu_{3}-\nu_{4}\right)+\delta \hat{\Gamma}\left(\nu_{3}-\nu_{4}\right)\right]\left[\bar{\Gamma} \delta\left(\nu_{1}\right)\right. \\
& \left.+\delta \hat{\Gamma}\left(\nu_{1}\right)\right]\left[\bar{\Gamma} \delta\left(\nu_{2}\right)+\delta \hat{\Gamma}^{*}\left(\nu_{2}\right)\right] \\
& +\left[\bar{\Gamma} \delta\left(\nu_{1}-\nu_{2}\right)+\delta \hat{\Gamma}\left(\nu_{1}-\nu_{2}\right)\right] \\
& \times\left[\bar{\Gamma} \delta\left(\nu_{3}-\nu_{4}\right)+\delta \hat{\Gamma}\left(\nu_{3}-\nu_{4}\right)\right] \\
& +\left[\bar{\Gamma} \delta\left(\nu_{1}+\nu_{3}\right)+\delta \hat{\Gamma}\left(\nu_{1}+\nu_{3}\right)\right] \\
& \times\left[\bar{\Gamma} \delta\left(\nu_{2}+\nu_{4}\right)+\delta \hat{\Gamma}^{*}\left(\nu_{2}+\nu_{4}\right)\right] \\
& +\left[\bar{\Gamma} \delta\left(\nu_{1}-\nu_{4}\right)+\delta \hat{\Gamma}\left(\nu_{1}-\nu_{4}\right)\right] \\
& \times\left[\bar{\Gamma} \delta\left(\nu_{2}-\nu_{3}\right)+\delta \hat{\Gamma}^{*}\left(\nu_{2}-\nu_{3}\right)\right] \\
& +\left[\bar{\Gamma} \delta\left(\nu_{1}-\nu_{2}+\nu_{3}\right)+\delta \hat{\Gamma}\left(\nu_{1}-\nu_{2}+\nu_{3}\right)\right] \\
& \times\left[\bar{\Gamma} \delta\left(\nu_{4}\right)+\delta \hat{\Gamma}^{*}\left(\nu_{4}\right)\right] \\
& +\left[\bar{\Gamma} \delta\left(\nu_{1}-\nu_{2}-\nu_{4}\right)+\delta \hat{\Gamma}\left(\nu_{1}-\nu_{2}-\nu_{4}\right)\right] \\
& \times\left[\bar{\Gamma} \delta\left(\nu_{3}\right)+\delta \hat{\Gamma}\left(\nu_{3}\right)\right] \\
& +\left[\bar{\Gamma} \delta\left(\nu_{1}+\nu_{3}-\nu_{4}\right)+\delta \hat{\Gamma}\left(\nu_{1}+\nu_{3}-\nu_{4}\right)\right] \\
& \times\left[\bar{\Gamma} \delta\left(\nu_{2}\right)+\delta \hat{\Gamma}^{*}\left(\nu_{2}\right)\right] \\
& +\left[\bar{\Gamma} \delta\left(-\nu_{2}+\nu_{3}-\nu_{4}\right)\right. \\
& \left.+\delta \hat{\Gamma}^{*}\left(\nu_{2}-\nu_{3}+\nu_{4}\right)\right] \\
& \times\left[\bar{\Gamma} \delta\left(\nu_{1}\right)+\delta \hat{\Gamma}\left(\nu_{1}\right)\right] \\
& +\bar{\Gamma} \delta\left(\nu_{1}-\nu_{2}+\nu_{3}-\nu_{4}\right) \\
& +\delta \hat{\Gamma}\left(\nu_{1}-\nu_{2}+\nu_{3}-\nu_{4}\right) \text {. }
\end{aligned}
$$

Averaging over the stationary process $\delta \Gamma(t)$ now involves evaluation of its third-order and fourth-order moments. However, these higher-order moments are not fully specified by the second moment, which is determined by the power spectrum given in Equation (58), because $\delta \Gamma(t)$ is not guaranteed to be Gaussian. Indeed, that $\delta \Gamma(t)$ is not Gaussian is shown in Appendix F. Nonetheless, $\delta \Gamma(t)$ may often be approximately Gaussian, and we proceed with this assumption recognizing that it may introduce small, detailed differences with the full quantum calculation. However, as could be anticipated, the term describing the sensitivity degradation due to photon bunching (labeled $\mathrm{C} 1 \mathrm{~b}$ below) involves only a second-order moment of $\delta \Gamma(t)$ and is therefore secure.

For a Gaussian $\delta \Gamma(t)$, and omitting the DC terms, we find

$$
\begin{aligned}
&\left\langle\hat{I}\left(\nu_{1}\right) \hat{I}^{*}\left(\nu_{2}\right) \hat{I}\left(\nu_{3}\right) \hat{I}^{*}\left(\nu_{4}\right)\right\rangle_{y, \delta \Gamma} \\
&=S_{\Gamma}\left(\nu_{1}\right) S_{\Gamma}\left(\nu_{3}\right) \delta\left(\nu_{1}-\nu_{2}\right) \delta\left(\nu_{3}-\nu_{4}\right) \\
&+S_{\Gamma}\left(\nu_{1}\right) S_{\Gamma}\left(\nu_{2}\right) \delta\left(\nu_{1}-\nu_{4}\right) \delta\left(\nu_{2}-\nu_{3}\right)(\mathrm{A} 1 \mathrm{~b}) \\
&+S_{\Gamma}\left(\nu_{1}\right) S_{\Gamma}\left(\nu_{4}\right) \delta\left(\nu_{1}+\nu_{3}\right) \delta\left(\nu_{2}+\nu_{4}\right)(\mathrm{A} 1 \mathrm{c}) \\
&+\bar{\Gamma} S_{\Gamma}\left(\nu_{3}\right) \delta\left(\nu_{1}-\nu_{2}\right) \delta\left(\nu_{3}-\nu_{4}\right) \\
&+\bar{\Gamma} S_{\Gamma}\left(\nu_{2}\right) \delta\left(\nu_{1}+\nu_{3}\right) \delta\left(\nu_{2}+\nu_{4}\right) \\
&+\bar{\Gamma} S_{\Gamma}\left(\nu_{2}\right) \delta\left(\nu_{1}-\nu_{4}\right) \delta\left(\nu_{2}-\nu_{3}\right) \\
&+\bar{\Gamma} S_{\Gamma}\left(\nu_{1}\right) \delta\left(\nu_{1}-\nu_{4}\right) \delta\left(\nu_{2}-\nu_{3}\right) \\
&+\bar{\Gamma} S_{\Gamma}\left(\nu_{1}\right) \delta\left(\nu_{1}+\nu_{3}\right) \delta\left(\nu_{2}+\nu_{4}\right) \\
&+\bar{\Gamma} S_{\Gamma}\left(\nu_{1}\right) \delta\left(\nu_{1}-\nu_{2}\right) \delta\left(\nu_{3}-\nu_{4}\right) \\
&+\bar{\Gamma}^{2} \delta\left(\nu_{1}-\nu_{2}\right) \delta\left(\nu_{3}-\nu_{4}\right) \\
&+\bar{\Gamma}^{2} \delta\left(\nu_{1}+\nu_{3}\right) \delta\left(\nu_{2}+\nu_{4}\right) \\
&+\bar{\Gamma}^{2} \delta\left(\nu_{1}-\nu_{4}\right) \delta\left(\nu_{2}-\nu_{3}\right) \\
&+\left[S_{\Gamma}\left(\nu_{1}-\nu_{2}\right)+S_{\Gamma}\left(\nu_{1}+\nu_{3}\right)\right. \\
&+S_{\Gamma}\left(\nu_{1}-\nu_{4}\right)+S_{\Gamma}\left(\nu_{4}\right)+S_{\Gamma}\left(\nu_{3}\right) \\
&\left.+S_{\Gamma}\left(\nu_{2}\right)+S_{\Gamma}\left(\nu_{1}\right)+\bar{\Gamma}\right] \\
& \times \delta\left(\nu_{1}-\nu_{2}+\nu_{3}-\nu_{4}\right)
\end{aligned}
$$

noting that the A1 term gives three contributions, A1a-A1c due to the Gaussian pairwise evaluation of the fourth-order moment of $\delta \Gamma$, while the factors in terms $\mathrm{C} 1-$ C3 combine to give two contributions each, e.g., C1a and $\mathrm{C} 1 \mathrm{~b}$.

We now evaluate the second moment of the shot noise intensity measure in the limit of a long measurement time, $\Delta \nu T \gg 1$. The result is

$$
\begin{aligned}
& \left\langle P_{W, T}^{2}\right\rangle \\
& =\int_{-\infty}^{+\infty} d \nu_{1} d \nu_{2} d \nu_{3} d \nu_{4} \\
& \quad \times\left\langle\hat{I}\left(\nu_{1}\right) \hat{I}^{*}\left(\nu_{2}\right) \hat{I}\left(\nu_{3}\right) \hat{I}^{*}\left(\nu_{4}\right)\right\rangle_{y, \delta \Gamma} \\
& \quad \times W\left(\nu_{1}\right) W^{*}\left(\nu_{2}\right) W\left(\nu_{3}\right) W^{*}\left(\nu_{4}\right)
\end{aligned}
$$




$$
\begin{aligned}
& \times \int_{-T / 2}^{+T / 2} d t e^{i 2 \pi\left(\nu_{1}-\nu_{2}\right) t} \\
& \times \int_{-T / 2}^{+T / 2} d t^{\prime} e^{i 2 \pi\left(\nu_{3}-\nu_{4}\right) t^{\prime}} \\
& =T^{2}\left[\int d \nu|W(\nu)|^{2} S_{\Gamma}(\nu)\right]^{2} \\
& +2 T \int d \nu|W(\nu)|^{4} S_{\Gamma}^{2}(\nu) \quad(\mathrm{A} 1 \mathrm{~b}+\mathrm{A} 1 \mathrm{c}) \\
& +2 T^{2} \bar{\Gamma} \int d \nu|W(\nu)|^{2} \\
& \times \int d \nu^{\prime}\left|W\left(\nu^{\prime}\right)\right|^{2} S_{\Gamma}\left(\nu^{\prime}\right) \quad(\mathrm{B} 1+\mathrm{B} 6) \\
& +4 T \bar{\Gamma} \int d \nu|W(\nu)|^{4} S_{\Gamma}(\nu)(\mathrm{B} 2+\mathrm{B} 3+\mathrm{B} 4+\mathrm{B} 5) \\
& +T^{2} \bar{\Gamma}^{2}\left[\int d \nu|W(\nu)|^{2}\right]^{2} \quad(\mathrm{C} 1 \mathrm{a}) \\
& +2 T \bar{\Gamma}^{2} \int d \nu|W(\nu)|^{4} \quad(\mathrm{C} 2 \mathrm{a}+\mathrm{C} 3 \mathrm{a}) \\
& +T S_{\Gamma}(0)\left[\int d \nu|W(\nu)|^{2}\right]^{2}(\mathrm{C} 1 \mathrm{~b}) \\
& +2 T \int d \nu d \nu^{\prime}|W(\nu)|^{2} \\
& \times\left|W\left(\nu^{\prime}\right)\right|^{2} S_{\Gamma}\left(\nu-\nu^{\prime}\right) \quad(\mathrm{C} 2 \mathrm{~b}+\mathrm{C} 3 \mathrm{~b}) \\
& +4 T \int d \nu|W(\nu)|^{2} \quad(\mathrm{C} 4 \mathrm{~b}+\mathrm{C} 5 \mathrm{~b} \\
& \left.\times \int d \nu^{\prime}\left|W\left(\nu^{\prime}\right)\right|^{2} S_{\Gamma}\left(\nu^{\prime}\right)+\mathrm{C} 6 \mathrm{~b}+\mathrm{C} 7 \mathrm{~b}\right) \\
& +T \bar{\Gamma}\left[\int d \nu|W(\nu)|^{2}\right]^{2} \text {. }
\end{aligned}
$$

The terms proportional to $T^{2}$ sum to give $\left\langle P_{W, T}\right\rangle^{2}$; the remaining terms proportional to $T$ give the variance

$$
\begin{aligned}
\sigma_{P}^{2}= & \left\langle P_{W, T}^{2}\right\rangle-\left\langle P_{W, T}\right\rangle^{2} & & \\
= & T\left\{2 \int d \nu|W(\nu)|^{4} S_{\Gamma}^{2}(\nu)\right. & & (\mathrm{A} 1 \mathrm{~b}+\mathrm{A} 1 \mathrm{c}) \\
& +4 \bar{\Gamma} \int d \nu|W(\nu)|^{4} S_{\Gamma}(\nu) & & (\mathrm{B} 2+\mathrm{B} 3+\mathrm{B} 4+\mathrm{B} 5) \\
& +2 \bar{\Gamma}^{2} \int d \nu|W(\nu)|^{4} & & (\mathrm{C} 2 \mathrm{a}+\mathrm{C} 3 \mathrm{a}) \\
& +S_{\Gamma}(0)\left[\int d \nu|W(\nu)|^{2}\right]^{2} & & (\mathrm{C} 1 \mathrm{~b}) \\
& +2 \int d \nu d \nu^{\prime}|W(\nu)|^{2} & & \\
& \times\left|W\left(\nu^{\prime}\right)\right|^{2} S_{\Gamma}\left(\nu-\nu^{\prime}\right) & & (\mathrm{C} 2 \mathrm{~b}+\mathrm{C} 3 \mathrm{~b}) \\
& +4 \int d \nu|W(\nu)|^{2} & & (\mathrm{C} 4 \mathrm{~b}+\mathrm{C} 5 \mathrm{~b} \\
& \times \int d \nu^{\prime}\left|W\left(\nu^{\prime}\right)\right|^{2} S_{\Gamma}\left(\nu^{\prime}\right) & & +\mathrm{C} 6 \mathrm{~b}+\mathrm{C} 7 \mathrm{~b}) \\
& \left.+\bar{\Gamma}\left[\int d \nu|W(\nu)|^{2}\right]^{2}\right\} . & & (\mathrm{D} 1)
\end{aligned}
$$

\section{APPENDIX C}

DETAILED EVALUATION OF SHOT NOISE

FLUCTUATIONS: MULTIPLE DETECTORS

Following the approach described in Appendix B, and omitting the DC terms, we have:

$$
\begin{aligned}
& \left\langle\hat{I}_{a 1}\left(\nu_{1}\right) \hat{I}_{a 2}^{*}\left(\nu_{2}\right) \hat{I}_{a 3}\left(\nu_{3}\right) \hat{I}_{a 4}^{*}\left(\nu_{4}\right)\right\rangle_{y} \\
& =\delta \hat{\Gamma}_{a 1}\left(\nu_{1}\right) \delta \hat{\Gamma}_{a 2}^{*}\left(\nu_{2}\right) \delta \hat{\Gamma}_{a 3}\left(\nu_{3}\right) \delta \hat{\Gamma}_{a 4}^{*}\left(\nu_{4}\right) \\
& +\delta_{a 1, a 2}\left[\bar{\Gamma}_{a 1} \delta\left(\nu_{1}-\nu_{2}\right)+\delta \hat{\Gamma}_{a 1}\left(\nu_{1}-\nu_{2}\right)\right] \\
& \times \delta \hat{\Gamma}_{a 3}\left(\nu_{3}\right) \delta \hat{\Gamma}_{a 4}^{*}\left(\nu_{4}\right) \\
& +\delta_{a 1, a 3}\left[\bar{\Gamma}_{a 1} \delta\left(\nu_{1}+\nu_{3}\right)+\delta \hat{\Gamma}_{a 1}\left(\nu_{1}+\nu_{3}\right)\right] \\
& \times \delta \hat{\Gamma}_{a 2}^{*}\left(\nu_{2}\right) \delta \hat{\Gamma}_{a 4}^{*}\left(\nu_{4}\right) \\
& +\delta_{a 1, a 4}\left[\bar{\Gamma}_{a 1} \delta\left(\nu_{1}-\nu_{4}\right)+\delta \hat{\Gamma}_{a 1}\left(\nu_{1}-\nu_{4}\right)\right] \\
& \times \delta \hat{\Gamma}_{a 2}^{*}\left(\nu_{2}\right) \delta \hat{\Gamma}_{a 3}\left(\nu_{3}\right) \\
& +\delta_{a 2, a 3}\left[\bar{\Gamma}_{a 2} \delta\left(\nu_{2}-\nu_{3}\right)+\delta \hat{\Gamma}_{a 2}\left(\nu_{2}-\nu_{3}\right)\right] \\
& \times \delta \hat{\Gamma}_{a 1}\left(\nu_{1}\right) \delta \hat{\Gamma}_{a 4}^{*}\left(\nu_{4}\right) \\
& +\delta_{a 2, a 4}\left[\bar{\Gamma}_{a 2} \delta\left(\nu_{2}+\nu_{4}\right)+\delta \hat{\Gamma}_{a 2}\left(\nu_{2}+\nu_{4}\right)\right] \\
& \times \delta \hat{\Gamma}_{a 1}\left(\nu_{1}\right) \delta \hat{\Gamma}_{a 3}\left(\nu_{3}\right) \\
& +\delta_{a 3, a 4}\left[\bar{\Gamma}_{a 3} \delta\left(\nu_{3}-\nu_{4}\right)+\delta \hat{\Gamma}_{a 3}\left(\nu_{3}-\nu_{4}\right)\right] \\
& \times \delta \hat{\Gamma}_{a 1}\left(\nu_{1}\right) \delta \hat{\Gamma}_{a 2}^{*}\left(\nu_{2}\right) \\
& +\delta_{a 1, a 2} \delta_{a 3, a 4}\left[\bar{\Gamma}_{a 1} \delta\left(\nu_{1}-\nu_{2}\right)+\delta \hat{\Gamma}_{a 1}\left(\nu_{1}-\nu_{2}\right)\right] \\
& \times\left[\bar{\Gamma}_{a 3} \delta\left(\nu_{3}-\nu_{4}\right)+\delta \hat{\Gamma}_{a 3}\left(\nu_{3}-\nu_{4}\right)\right] \\
& +\delta_{a 1, a 3} \delta_{a 2, a 4}\left[\bar{\Gamma}_{a 1} \delta\left(\nu_{1}+\nu_{3}\right)+\delta \hat{\Gamma}_{a 1}\left(\nu_{1}+\nu_{3}\right)\right] \\
& \times\left[\bar{\Gamma}_{a 2} \delta\left(\nu_{2}+\nu_{4}\right)+\delta \hat{\Gamma}_{a 2}^{*}\left(\nu_{2}+\nu_{4}\right)\right] \\
& +\delta_{a 1, a 4} \delta_{a 2, a 3}\left[\bar{\Gamma}_{a 1} \delta\left(\nu_{1}-\nu_{4}\right)+\delta \hat{\Gamma}_{a 1}\left(\nu_{1}-\nu_{4}\right)\right] \\
& \times\left[\bar{\Gamma}_{a 2} \delta\left(\nu_{2}-\nu_{3}\right)+\delta \hat{\Gamma}_{a 2}^{*}\left(\nu_{2}-\nu_{3}\right)\right] \\
& +\delta_{a 1, a 2} \delta_{a 1, a 3}\left[\bar{\Gamma}_{a 1} \delta\left(\nu_{1}-\nu_{2}+\nu_{3}\right)\right. \\
& \left.+\delta \hat{\Gamma}_{a 1}\left(\nu_{1}-\nu_{2}+\nu_{3}\right)\right] \delta \hat{\Gamma}_{a 4}^{*}\left(\nu_{4}\right) \\
& +\delta_{a 1, a 2} \delta_{a 1, a 4}\left[\bar{\Gamma}_{a 1} \delta\left(\nu_{1}-\nu_{2}-\nu_{4}\right)\right. \\
& \left.+\delta \hat{\Gamma}_{a 1}\left(\nu_{1}-\nu_{2}-\nu_{4}\right)\right] \delta \hat{\Gamma}_{a 3}\left(\nu_{3}\right) \\
& +\delta_{a 1, a 3} \delta_{a 1, a 4}\left[\bar{\Gamma}_{a 1} \delta\left(\nu_{1}+\nu_{3}-\nu_{4}\right)\right. \\
& \left.+\delta \hat{\Gamma}_{a 1}\left(\nu_{1}+\nu_{3}-\nu_{4}\right)\right] \delta \hat{\Gamma}_{a 2}^{*}\left(\nu_{2}\right) \\
& +\delta_{a 2, a 3} \delta_{a 2, a 4}\left[\bar{\Gamma}_{a 2} \delta\left(-\nu_{2}+\nu_{3}-\nu_{4}\right)\right. \\
& \left.+\delta \hat{\Gamma}_{a 2}^{*}\left(\nu_{2}-\nu_{3}+\nu_{4}\right)\right] \delta \hat{\Gamma}_{a 1}\left(\nu_{1}\right) \\
& +\delta_{a 1, a 2} \delta_{a 1, a 3} \delta_{a 1, a 4} \bar{\Gamma}_{a 1} \delta\left(\nu_{1}-\nu_{2}+\nu_{3}-\nu_{4}\right) \\
& +\delta \hat{\Gamma}_{a 1}\left(\nu_{1}-\nu_{2}+\nu_{3}-\nu_{4}\right) \text {. }
\end{aligned}
$$


Inserting this result into Equation (92) and evaluating some integrals, we find

$$
\begin{aligned}
& F_{a b c d} \\
& =T^{2} \int d \nu|W(\nu)|^{2} C_{a b}^{(\Gamma)}(\nu) \\
& \times \int d \nu^{\prime}\left|W\left(\nu^{\prime}\right)\right|^{2} C_{c d}^{(\Gamma)}\left(\nu^{\prime}\right) \\
& +T \int d \nu|W(\nu)|^{4}\left[C_{a c}^{(\Gamma)}(\nu) C_{d b}^{(\Gamma)}(\nu)\right. \\
& \left.+C_{a d}^{(\Gamma)}(\nu) C_{c b}^{(\Gamma)}(\nu)\right] \\
& +T^{2} \int d \nu|W(\nu)|^{2} \\
& \times \int d \nu^{\prime}\left|W\left(\nu^{\prime}\right)\right|^{2} \\
& \times\left[\delta_{a b} \bar{\Gamma}_{a} C_{c d}^{(\Gamma)}\left(\nu^{\prime}\right)+\delta_{c d} \bar{\Gamma}_{c} C_{a b}^{(\Gamma)}\left(\nu^{\prime}\right)\right] \\
& +T \int d \nu|W(\nu)|^{4}\left[\delta_{a c} \bar{\Gamma}_{a} C_{d b}^{(\Gamma)}(\nu)\right. \\
& +\delta_{a d} \bar{\Gamma}_{a} C_{c b}^{(\Gamma)}(\nu)+\delta_{b c} \bar{\Gamma}_{b} C_{a d}^{(\Gamma)}(\nu) \\
& \left.+\delta_{b d} \bar{\Gamma}_{b} C_{c a}^{(\Gamma)}(\nu)\right] \\
& +T^{2} \delta_{a b} \delta_{c d} \bar{\Gamma}_{a} \bar{\Gamma}_{c}\left[\int d \nu|W(\nu)|^{2}\right]^{2} \\
& +T\left[\delta_{a c} \delta_{b d} \bar{\Gamma}_{a} \bar{\Gamma}_{b}+\delta_{a d} \delta_{b c} \bar{\Gamma}_{a} \bar{\Gamma}_{c}\right] \\
& \times \int d \nu|W(\nu)|^{4} \\
& +T \delta_{a b} \delta_{c d} C_{a c}^{(\Gamma)}(0)\left[\int d \nu|W(\nu)|^{2}\right]^{2} \\
& +T \int d \nu d \nu^{\prime}|W(\nu)|^{2}\left|W\left(\nu^{\prime}\right)\right|^{2} \\
& \times\left[\delta_{a c} \delta_{b d}+\delta_{a d} \delta_{b c}\right] C_{a b}^{(\Gamma)}\left(\nu-\nu^{\prime}\right) \\
& +T \int d \nu|W(\nu)|^{2} \int d \nu^{\prime}\left|W\left(\nu^{\prime}\right)\right|^{2} \\
& \times\left[\delta_{a b} \delta_{a c} C_{a d}^{(\Gamma)}\left(\nu^{\prime}\right)+\delta_{a b} \delta_{a d} C_{a c}^{(\Gamma)}\left(\nu^{\prime}\right)\right. \\
& \left.+\delta_{a c} \delta_{a d} C_{a b}^{(\Gamma)}\left(\nu^{\prime}\right)+\delta_{b c} \delta_{b d} C_{a b}^{(\Gamma)}\left(\nu^{\prime}\right)\right] \\
& +T \delta_{a b} \delta_{a c} \delta_{a d} \bar{\Gamma}_{a}\left[\int d \nu|W(\nu)|^{2}\right]^{2} . \\
& (\mathrm{A} 1 \mathrm{~b}+\mathrm{A} 1 \mathrm{c}) \\
& (\mathrm{B} 1+\mathrm{B} 6) \\
& (\mathrm{B} 2+\mathrm{B} 3 \\
& +\mathrm{B} 4+\mathrm{B} 5) \\
& (\mathrm{C} 2 \mathrm{a}+\mathrm{C} 3 \mathrm{a}) \\
& (C 2 b+C 3 b) \\
& (C 4 b+C 5 b \\
& +\mathrm{C} 6 \mathrm{~b}+\mathrm{C} 7 \mathrm{~b})
\end{aligned}
$$

The fluctuations of the noise intensity of the difference current are obtained by considering

$$
\begin{aligned}
\left\langle P_{\Delta}^{2}\right\rangle & =F_{1111}+F_{2222}+4 F_{1212}+2 F_{1122}-4 F_{1112}-4 F_{2212} \\
& =2 F_{1111}+2 F_{1122}+4 F_{1212}-8 F_{1112},
\end{aligned}
$$

as can be seen by computing $P_{\Delta}^{2}$ (Equation (88)) and making use of the symmetry of the $50 / 50$ beamsplitter. The $T^{2}$ terms are eliminated by subtracting the square of the mean,

$$
\begin{aligned}
\sigma_{P_{\Delta}}^{2}= & \left\langle P_{\Delta}^{2}\right\rangle-\left\langle P_{\Delta}\right\rangle^{2}=2 F_{1111}+2 F_{1122} \\
& +4 F_{1212}-8 F_{1112}
\end{aligned}
$$

$$
\begin{aligned}
& =4 T \int d \nu|W(\nu)|^{4}\left[C_{11}^{(\Gamma)}(\nu)\right]^{2} \\
& +8 T \bar{\Gamma}_{1} \int d \nu|W(\nu)|^{4} C_{11}^{(\Gamma)}(\nu) \\
& +4 T \bar{\Gamma}_{1}^{2} \int d \nu|W(\nu)|^{4}+2 T C_{11}^{(\Gamma)}(0) \\
& \times\left[\int d \nu|W(\nu)|^{2}\right]^{2} \\
& +4 T \int d \nu d \nu^{\prime}|W(\nu)|^{2} \\
& \times\left|W\left(\nu^{\prime}\right)\right|^{2} C_{11}^{(\Gamma)}\left(\nu-\nu^{\prime}\right) \\
& +8 T \int d \nu d \nu^{\prime}|W(\nu)|^{2} \\
& \times\left|W\left(\nu^{\prime}\right)\right|^{2} C_{11}^{(\Gamma)}\left(\nu^{\prime}\right) \\
& +2 T \bar{\Gamma}_{1}\left[\int d \nu|W(\nu)|^{2}\right]^{2} \\
& +4 T \int d \nu|W(\nu)|^{4}\left[C_{11}^{(\Gamma)}(\nu)\right]^{2} \\
& +2 T C_{11}^{(\Gamma)}(0)\left[\int d \nu|W(\nu)|^{2}\right]^{2} \\
& +8 T \int d \nu|W(\nu)|^{4}\left[C_{11}^{(\Gamma)}(\nu)\right]^{2} \\
& +8 T \bar{\Gamma}_{1} \int d \nu|W(\nu)|^{4} C_{11}^{(\Gamma)}(\nu) \\
& +4 T \bar{\Gamma}_{1}^{2} \int d \nu|W(\nu)|^{4} \\
& +4 T \int d \nu d \nu^{\prime}|W(\nu)|^{2} \\
& \times\left|W\left(\nu^{\prime}\right)\right|^{2} C_{11}^{(\Gamma)}\left(\nu-\nu^{\prime}\right) \\
& -16 T \int d \nu|W(\nu)|^{4}\left[C_{11}^{(\Gamma)}\right]^{2}(\nu) \\
& -16 T \bar{\Gamma}_{1} \int d \nu|W(\nu)|^{4} C_{11}^{(\Gamma)}(\nu) \\
& -8 T \int d \nu d \nu^{\prime}|W(\nu)|^{2} \\
& \times\left|W\left(\nu^{\prime}\right)\right|^{2} C_{11}^{(\Gamma)}\left(\nu^{\prime}\right) \quad\left(-8 F_{1112}\right) .
\end{aligned}
$$

The resulting sum is

$$
\begin{aligned}
\sigma_{P_{\Delta}}^{2}= & +8 T \bar{\Gamma}_{1}^{2} \int d \nu|W(\nu)|^{4}+4 T C_{11}^{(\Gamma)}(0)\left[\int d \nu|W(\nu)|^{2}\right]^{2} \\
& +8 T \int d \nu d \nu^{\prime}|W(\nu)|^{2}\left|W\left(\nu^{\prime}\right)\right|^{2} C_{11}^{(\Gamma)}\left(\nu-\nu^{\prime}\right) \\
& +2 T \bar{\Gamma}_{1}\left[\int d \nu|W(\nu)|^{2}\right]^{2} .
\end{aligned}
$$

\section{APPENDIX D}

\section{QUANTUM CALCULATION: EVALUATION OF EIGHTH-ORDER MOMENTS}

In this appendix, we evaluate eighth-order moments of the photon operators that are needed for a quantum-mechanical calculation of the sensitivity of a shot-noise measurement scheme, as outlined in Section 7 and Equation (98). As described in that section, there are nine operator permutations that give nonvanishing contributions out of the $4 !=24$ 
possibilities. We will not evaluate all of these terms but instead choose a few that are instructive, including the term that is responsible for the sensitivity degradation due to photon bunching.

We start with the $2^{2}$ permutations, (12)(34), (13)(24), and (14)(23):

$$
\begin{aligned}
\text { (12)(34) }= & \left\langle b^{\dagger}\left(\nu_{1}^{\prime}\right) b\left(\nu_{2}^{\prime}\right)\right\rangle\left\langle b\left(\nu_{1}^{\prime}+\nu_{1}\right) b^{\dagger}\left(\nu_{2}^{\prime}+\nu_{2}\right)\right\rangle \\
& \times\left\langle b^{\dagger}\left(\nu_{3}^{\prime}\right) b\left(\nu_{4}^{\prime}\right)\right\rangle\left\langle b\left(\nu_{3}^{\prime}+\nu_{3}\right) b^{\dagger}\left(\nu_{4}^{\prime}+\nu_{4}\right)\right\rangle .
\end{aligned}
$$

Performing the indicated averages and integrations gives

$$
\begin{aligned}
\text { (12) } & (34)=\int d \nu_{1}^{\prime} d \nu_{2}^{\prime} d \nu_{3}^{\prime} d \nu_{4}^{\prime}\left\{n\left(\nu_{1}^{\prime}\right) \delta\left(\nu_{1}^{\prime}-\nu_{2}^{\prime}\right)\right. \\
\times & \left.\times n\left(\nu_{1}^{\prime}+\nu_{1}\right)+1\right] \\
\times & \delta\left(\nu_{1}^{\prime}+\nu_{1}-\nu_{2}^{\prime}-\nu_{2}\right) n\left(\nu_{3}^{\prime}\right) \delta\left(\nu_{3}^{\prime}-\nu_{4}^{\prime}\right) \\
\times & \left.\times\left[n\left(\nu_{3}^{\prime}+\nu_{3}\right)+1\right] \delta\left(\nu_{3}^{\prime}+\nu_{3}-\nu_{4}^{\prime}-\nu_{4}\right)\right\} \\
= & \int d \nu_{1}^{\prime} d \nu_{3}^{\prime} n\left(\nu_{1}^{\prime}\right)\left[n\left(\nu_{1}^{\prime}+\nu_{1}\right)+1\right] \delta\left(\nu_{1}-\nu_{2}\right) \\
& \times n\left(\nu_{3}^{\prime}\right)\left[n\left(\nu_{3}^{\prime}+\nu_{3}\right)+1\right] \delta\left(\nu_{3}-\nu_{4}\right) \\
= & {\left[\bar{\Gamma}+S_{\Gamma}\left(\nu_{1}\right)\right]\left[\bar{\Gamma}+S_{\Gamma}\left(\nu_{3}\right)\right] } \\
& \times \delta\left(\nu_{1}-\nu_{2}\right) \delta\left(\nu_{3}-\nu_{4}\right) .
\end{aligned}
$$

Comparison with the semiclassical calculation detailed in Appendix B shows that we have reproduced the terms A1a, B1, $\mathrm{B} 6$, and $\mathrm{C} 1 \mathrm{a}$; these become proportional to $T^{2}$ after the integrations over time and are related to the mean value of $P_{W, T}$ rather than its fluctuations. Next, we evaluate

$$
\begin{aligned}
\text { (13) (24) }= & \left\langle b^{\dagger}\left(\nu_{1}^{\prime}\right) b\left(\nu_{3}^{\prime}+\nu_{3}\right)\right\rangle\left\langle b\left(\nu_{1}^{\prime}+\nu_{1}\right) b^{\dagger}\left(\nu_{3}^{\prime}\right)\right\rangle \\
& \times\left\langle b^{\dagger}\left(\nu_{2}^{\prime}+\nu_{2}\right) b\left(\nu_{4}^{\prime}\right)\right\rangle\left\langle b\left(\nu_{2}^{\prime}\right) b^{\dagger}\left(\nu_{4}^{\prime}+\nu_{4}\right)\right\rangle .
\end{aligned}
$$

Upon averaging and integrating,

$$
\begin{aligned}
(13)(24)= & \int d \nu_{1}^{\prime} d \nu_{2}^{\prime} d \nu_{3}^{\prime} d \nu_{4}^{\prime}\left\{n\left(\nu_{1}^{\prime}\right) \delta\left(\nu_{1}^{\prime}-\nu_{3}^{\prime}-\nu_{3}\right)\right. \\
& \times\left[n\left(\nu_{1}^{\prime}+\nu_{1}\right)+1\right] \delta\left(\nu_{3}^{\prime}-\nu_{1}^{\prime}-\nu_{1}\right) \\
& \times n\left(\nu_{4}^{\prime}\right) \delta\left(\nu_{4}^{\prime}-\nu_{2}^{\prime}-\nu_{2}\right)\left[n\left(\nu_{4}^{\prime}+\nu_{4}\right)+1\right] \\
& \left.\times \delta\left(\nu_{2}^{\prime}-\nu_{4}^{\prime}-\nu_{4}\right)\right\} \\
= & \int d \nu_{1}^{\prime} d \nu_{4}^{\prime}\left\{n ( \nu _ { 1 } ^ { \prime } ) [ n ( \nu _ { 1 } ^ { \prime } + \nu _ { 1 } ) + 1 ] \delta \left(\nu_{1}+\nu_{3}^{\prime},\right.\right. \\
& \left.\times n\left(\nu_{4}^{\prime}\right)\left[n\left(\nu_{4}^{\prime}+\nu_{4}\right)+1\right] \delta\left(\nu_{2}+\nu_{4}\right)\right\} \\
= & {\left[\bar{\Gamma}+S_{\Gamma}\left(\nu_{1}\right)\right]\left[\bar{\Gamma}+S_{\Gamma}\left(\nu_{2}\right)\right] } \\
& \times \delta\left(\nu_{1}+\nu_{3}\right) \delta\left(\nu_{2}+\nu_{4}\right) .
\end{aligned}
$$

Comparison with the semiclassical calculation shows that we have reproduced the terms A1c, B2, B5, and $\mathrm{C} 2 \mathrm{a}$; these become proportional to $T$ after the integrations over time and are therefore related to the fluctuations of $P_{W, T}$. Similarly,

$$
\begin{aligned}
(14)(23)= & {\left[\bar{\Gamma}+S_{\Gamma}\left(\nu_{1}\right)\right]\left[\bar{\Gamma}+S_{\Gamma}\left(\nu_{2}\right)\right] } \\
& \times \delta\left(\nu_{1}-\nu_{4}\right) \delta\left(\nu_{2}-\nu_{3}\right)
\end{aligned}
$$

corresponds to the semiclassical terms A1b, B3, B4, and C3a, which again are fluctuation terms since they are proportional to $T$.

We now turn to the $4^{1}$ terms: (1234), (1243), (1324), (1342), (1423), (1432). The terms that we have derived so far using the $2^{2}$ permutations reproduce the semiclassical results of Section 5 and represent $1 / B T$ noise, or terms that vanish if we choose a noise filter $W(\nu)$ that rejects the bunching noise component at low frequency illustrated in Figure 3. The $4^{1}$ permutations are more interesting. We start with

$$
\begin{aligned}
(1234)= & \left\langle b^{\dagger}\left(\nu_{1}^{\prime}\right) b\left(\nu_{2}^{\prime}\right)\right\rangle\left\langle b^{\dagger}\left(\nu_{2}^{\prime}+\nu_{2}\right) b\left(\nu_{3}^{\prime}+\nu_{3}\right)\right\rangle \\
& \times\left\langle b^{\dagger}\left(\nu_{3}^{\prime}\right) b\left(\nu_{4}^{\prime}\right)\right\rangle\left\langle b\left(\nu_{1}^{\prime}+\nu_{1}\right) b^{\dagger}\left(\nu_{4}^{\prime}+\nu_{4}\right)\right\rangle .
\end{aligned}
$$

Averaging and integrating,

$$
\begin{aligned}
(1234)= & \int d \nu_{1}^{\prime} d \nu_{2}^{\prime} d \nu_{3}^{\prime} d \nu_{4}^{\prime}\left\{n\left(\nu_{1}^{\prime}\right) \delta\left(\nu_{1}^{\prime}-\nu_{2}^{\prime}\right)\right. \\
& n\left(\nu_{2}^{\prime}+\nu_{2}\right) \delta\left(\nu_{2}^{\prime}+\nu_{2}-\nu_{3}^{\prime}-\nu_{3}\right) \\
& \times n\left(\nu_{3}^{\prime}\right) \delta\left(\nu_{3}^{\prime}-\nu_{4}^{\prime}\right)\left[n\left(\nu_{4}^{\prime}+\nu_{4}\right)+1\right] \\
& \left.\times \delta\left(\nu_{1}^{\prime}+\nu_{1}-\nu_{4}^{\prime}-\nu_{4}\right)\right\} \\
= & \int d \nu_{1}^{\prime} d \nu_{3}^{\prime}\left\{n\left(\nu_{1}^{\prime}\right) n\left(\nu_{1}^{\prime}+\nu_{2}\right) n\left(\nu_{3}^{\prime}\right)\right. \\
& \times\left[n\left(\nu_{3}^{\prime}+\nu_{4}\right)+1\right] \\
& \left.\times \delta\left(\nu_{1}^{\prime}-\nu_{3}^{\prime}+\nu_{2}-\nu_{3}\right) \delta\left(\nu_{1}^{\prime}-\nu_{3}^{\prime}+\nu_{1}-\nu_{4}\right)\right\} \\
= & \int d \nu_{1}^{\prime} n\left(\nu_{1}^{\prime}\right) n\left(\nu_{1}^{\prime}+\nu_{2}\right) n\left(\nu_{1}^{\prime}+\nu_{1}-\nu_{4}\right) \\
& \times\left[n\left(\nu_{1}^{\prime}+\nu_{1}\right)+1\right] \\
& \times \delta\left(\nu_{1}-\nu_{2}+\nu_{3}-\nu_{4}\right) .
\end{aligned}
$$

This represents a contribution to the shot noise fluctuations given by

$$
\begin{aligned}
\left\langle P_{W, T}^{2}\right\rangle_{(1234)}= & \int_{-\infty}^{+\infty} d \nu_{1} d \nu_{2} d \nu_{3} d \nu_{4} W\left(\nu_{1}\right) \\
& \times W^{*}\left(\nu_{2}\right) W\left(\nu_{3}\right) W^{*}\left(\nu_{4}\right) \\
& \times F_{(1234)}^{\prime}\left(\nu_{1}, \nu_{2}, \nu_{3}, \nu_{4}\right) \delta\left(\nu_{1}-\nu_{2}+\nu_{3}-\nu_{4}\right) \\
& \times \int_{-T / 2}^{+T / 2} d t e^{i 2 \pi\left(\nu_{1}-\nu_{2}\right) t} \int_{-T / 2}^{+T / 2} d t^{\prime} e^{i 2 \pi\left(\nu_{3}-\nu_{4}\right) t^{\prime}} \\
= & T \int_{-\infty}^{+\infty} d \nu_{1} d \nu_{3}\left|W\left(\nu_{1}\right)\right|^{2}\left|W\left(\nu_{3}\right)\right|^{2} \\
& \times \int d \nu_{1}^{\prime} n\left(\nu_{1}^{\prime}\right) n\left(\nu_{1}^{\prime}+\nu_{1}\right) n\left(\nu_{1}^{\prime}+\nu_{1}-\nu_{3}\right) \\
& \times\left[n\left(\nu_{1}^{\prime}+\nu_{1}\right)+1\right] .
\end{aligned}
$$

This term did not appear in the semiclassical analysis, and presumably represents non-gaussianity of the photon arrival rate fluctuations which are expected in the quantum calculation as shown in Appendix F but are neglected in Section 5. However, for this term to be appreciable, the noise frequencies $\nu_{1}$ and $\nu_{3}$ must be comparable to or smaller than the optical bandwidth $\Delta \nu$; this term does not contribute if we choose a cutoff for $W(\nu)$ that is well above $\Delta \nu$ as shown in Figure 3. 
Next is the pairing

$$
\begin{aligned}
(1243)= & \left\langle b^{\dagger}\left(\nu_{1}^{\prime}\right) b\left(\nu_{2}^{\prime}\right)\right\rangle\left\langle b^{\dagger}\left(\nu_{2}^{\prime}+\nu_{2}\right) b\left(\nu_{4}^{\prime}\right)\right\rangle \\
& \times\left\langle b\left(\nu_{1}^{\prime}+\nu_{1}\right) b^{\dagger}\left(\nu_{3}^{\prime}\right)\right\rangle\left\langle b\left(\nu_{3}^{\prime}+\nu_{3}\right) b^{\dagger}\left(\nu_{4}^{\prime}+\nu_{4}\right)\right\rangle .
\end{aligned}
$$

Averaging and integrating,

$$
\begin{aligned}
(1243)= & \int d \nu_{1}^{\prime} d \nu_{2}^{\prime} d \nu_{3}^{\prime} d \nu_{4}^{\prime}\left\{n\left(\nu_{1}^{\prime}\right) \delta\left(\nu_{1}^{\prime}-\nu_{2}^{\prime}\right)\right. \\
& \times n\left(\nu_{2}^{\prime}+\nu_{2}\right) \delta\left(\nu_{4}^{\prime}-\nu_{2}^{\prime}-\nu_{2}\right) \\
& \times\left[n\left(\nu_{1}^{\prime}+\nu_{1}\right)+1\right] \delta\left(\nu_{3}^{\prime}-\nu_{1}^{\prime}-\nu_{1}\right) \\
& \left.\times\left[n\left(\nu_{3}^{\prime}+\nu_{3}\right)+1\right] \delta\left(\nu_{4}^{\prime}+\nu_{4}-\nu_{3}^{\prime}-\nu_{3}\right)\right\} \\
= & \int d \nu_{1}^{\prime} n\left(\nu_{1}^{\prime}\right) n\left(\nu_{1}^{\prime}+\nu_{2}\right)\left[1+n\left(\nu_{1}^{\prime}+\nu_{1}\right)\right] \\
& \times\left[1+n\left(\nu_{1}^{\prime}+\nu_{2}+\nu_{4}\right)\right] \delta\left(\nu_{1}-\nu_{2}+\nu_{3}-\nu_{4}\right) .
\end{aligned}
$$

This term contributes a shot noise fluctuation given by

$$
\begin{aligned}
\left\langle P_{W, T}^{2}\right\rangle_{(1243)}= & T \int_{-\infty}^{+\infty} d \nu_{1} d \nu_{3}\left|W\left(\nu_{1}\right)\right|^{2}\left|W\left(\nu_{3}\right)\right|^{2} \\
& \times \int d \nu_{1}^{\prime} n\left(\nu_{1}^{\prime}\right) n\left(\nu_{1}^{\prime}+\nu_{1}\right)\left[1+n\left(\nu_{1}^{\prime}+\nu_{1}\right)\right] \\
& \times\left[1+n\left(\nu_{1}^{\prime}+\nu_{1}+\nu_{3}\right)\right]
\end{aligned}
$$

Again, this term is small if we chose the high-pass filter cutoff frequency well above the optical bandwidth $\Delta \nu$. Note that there is a contribution

$$
\begin{gathered}
T \int_{-\infty}^{+\infty} d \nu_{1} d \nu_{3}\left|W\left(\nu_{1}\right)\right|^{2}\left|W\left(\nu_{3}\right)\right|^{2} \int d \nu_{1}^{\prime} n\left(\nu_{1}^{\prime}\right) n\left(\nu_{1}^{\prime}+\nu_{1}\right) \\
\quad=T \int_{-\infty}^{+\infty} d \nu_{1}\left|W\left(\nu_{1}\right)\right|^{2} S_{\Gamma}\left(\nu_{1}\right) \int_{-\infty}^{+\infty} d \nu_{3}\left|W\left(\nu_{3}\right)\right|^{2}
\end{gathered}
$$

that reproduces the semiclassical term C7. We skip ahead and look at

$$
\begin{aligned}
(1432)= & \left\langle b^{\dagger}\left(\nu_{1}^{\prime}\right) b\left(\nu_{4}^{\prime}\right)\right\rangle\left\langle b\left(\nu_{1}^{\prime}+\nu_{1}\right) b^{\dagger}\left(\nu_{2}^{\prime}+\nu_{2}\right)\right\rangle \\
& \times\left\langle b\left(\nu_{2}^{\prime}\right) b^{\dagger}\left(\nu_{3}^{\prime}\right)\right\rangle\left\langle b\left(\nu_{3}^{\prime}+\nu_{3}\right) b^{\dagger}\left(\nu_{4}^{\prime}+\nu_{4}\right)\right\rangle .
\end{aligned}
$$

Averaging and integrating,

$$
\begin{aligned}
(1432)= & \int d \nu_{1}^{\prime} d \nu_{2}^{\prime} d \nu_{3}^{\prime} d \nu_{4}^{\prime}\left\{n\left(\nu_{1}^{\prime}\right) \delta\left(\nu_{1}^{\prime}-\nu_{4}^{\prime}\right)\right. \\
& \times\left[1+n\left(\nu_{1}^{\prime}+\nu_{1}\right)\right] \delta\left(\nu_{1}^{\prime}+\nu_{1}-\nu_{2}^{\prime}-\nu_{2}\right) \\
& \times\left[1+n\left(\nu_{2}^{\prime}\right)\right] \delta\left(\nu_{2}^{\prime}-\nu_{3}^{\prime}\right)\left[1+n\left(\nu_{3}^{\prime}+\nu_{3}\right)\right] \\
& \left.\times \delta\left(\nu_{4}^{\prime}+\nu_{4}-\nu_{3}^{\prime}-\nu_{3}\right)\right\} \\
= & \int d \nu_{1}^{\prime} n\left(\nu_{1}^{\prime}\right)\left[1+n\left(\nu_{1}^{\prime}+\nu_{1}\right)\right]\left[1+n\left(\nu_{1}^{\prime}+\nu_{1}-\nu_{2}\right)\right] \\
& \times\left[1+n\left(\nu_{1}^{\prime}+\nu_{4}\right)\right] \\
& \times \delta\left(\nu_{1}-\nu_{2}+\nu_{3}-\nu_{4}\right) .
\end{aligned}
$$

The product expands to eight terms. The first term is

$$
\begin{gathered}
\int d \nu_{1}^{\prime} n\left(\nu_{1}^{\prime}\right) \delta\left(\nu_{1}-\nu_{2}+\nu_{3}-\nu_{4}\right) \\
=\bar{\Gamma} \delta\left(\nu_{1}-\nu_{2}+\nu_{3}-\nu_{4}\right)
\end{gathered}
$$

and reproduces the semiclassical term responsible for Poisson noise, D1. Another term is

$$
\begin{aligned}
& \int d \nu_{1}^{\prime} n\left(\nu_{1}^{\prime}\right) n\left(\nu_{1}^{\prime}+\nu_{1}-\nu_{2}\right) \\
& \delta\left(\nu_{1}-\nu_{2}+\nu_{3}-\nu_{4}\right) \\
&= S_{\Gamma}\left(\nu_{1}-\nu_{2}\right) \delta\left(\nu_{1}-\nu_{2}+\nu_{3}-\nu_{4}\right)
\end{aligned}
$$

and reproduces the semiclassical term $\mathrm{C} 1 \mathrm{~b}$ in Appendix B.

For the case of multiple detectors, the photon operators are decorated with a subscript to indicate the detector that they correspond to. Thus, we are interested in

$$
\begin{aligned}
(1432)= & \left\langle b_{a}^{\dagger}\left(\nu_{1}^{\prime}\right) b_{d}\left(\nu_{4}^{\prime}\right)\right\rangle\left\langle b_{a}\left(\nu_{1}^{\prime}+\nu_{1}\right) b_{b}^{\dagger}\left(\nu_{2}^{\prime}+\nu_{2}\right)\right\rangle \\
& \times\left\langle b_{b}\left(\nu_{2}^{\prime}\right) b_{c}^{\dagger}\left(\nu_{3}^{\prime}\right)\right\rangle\left\langle b_{c}\left(\nu_{3}^{\prime}+\nu_{3}\right) b_{d}^{\dagger}\left(\nu_{4}^{\prime}+\nu_{4}\right)\right\rangle .
\end{aligned}
$$

Averaging and integrating,

$$
\begin{aligned}
(1432)= & \int d \nu_{1}^{\prime} d \nu_{2}^{\prime} d \nu_{3}^{\prime} d \nu_{4}^{\prime}\left\{B_{a d}\left(\nu_{1}^{\prime}\right) \delta\left(\nu_{1}^{\prime}-\nu_{4}^{\prime}\right)\right. \\
& \times\left[\delta_{a b}+B_{b a}\left(\nu_{1}^{\prime}+\nu_{1}\right)\right] \delta\left(\nu_{1}^{\prime}+\nu_{1}-\nu_{2}^{\prime}-\nu_{2}\right) \\
& \times\left[\delta_{b c}+B_{c b}\left(\nu_{2}^{\prime}\right)\right] \delta\left(\nu_{2}^{\prime}-\nu_{3}^{\prime}\right)\left[\delta_{c d}+B_{d c}\left(\nu_{3}^{\prime}+\nu_{3}\right)\right] \\
& \left.\times \delta\left(\nu_{4}^{\prime}+\nu_{4}-\nu_{3}^{\prime}-\nu_{3}\right)\right\} \\
= & \int d \nu_{1}^{\prime} B_{a d}\left(\nu_{1}^{\prime}\right)\left[\delta_{a b}+B_{b a}\left(\nu_{1}^{\prime}+\nu_{1}\right)\right] \\
& \times\left[\delta_{b c}+B_{c b}\left(\nu_{1}^{\prime}+\nu_{1}-\nu_{2}\right)\right]\left[\delta_{c d}+B_{d c}\left(\nu_{1}^{\prime}+\nu_{4}\right)\right] \\
& \times \delta\left(\nu_{1}-\nu_{2}+\nu_{3}-\nu_{4}\right) .
\end{aligned}
$$

Again there are eight terms. The first term is

$$
\begin{gathered}
\delta_{a b} \delta_{b c} \delta_{c d} \int d \nu_{1}^{\prime} B_{a d}\left(\nu_{1}^{\prime}\right) \delta\left(\nu_{1}-\nu_{2}+\nu_{3}-\nu_{4}\right) \\
=\delta_{a b} \delta_{a c} \delta_{a d} \bar{\Gamma}_{a} \delta\left(\nu_{1}-\nu_{2}+\nu_{3}-\nu_{4}\right)
\end{gathered}
$$

and reproduces the semiclassical D1 term in Appendix C that is responsible for Poisson noise. The interesting term is

$$
\begin{aligned}
& \delta_{a b} \delta_{c d} \\
& \qquad d \nu_{1}^{\prime} B_{a d}\left(\nu_{1}^{\prime}\right) B_{c b}\left(\nu_{1}^{\prime}+\nu_{1}-\nu_{2}\right) \delta\left(\nu_{1}-\nu_{2}+\nu_{3}-\nu_{4}\right) \\
& \quad=\delta_{a b} \delta_{c d} C_{a c}^{(\Gamma)}\left(\nu_{1}-\nu_{2}\right) \delta\left(\nu_{1}-\nu_{2}+\nu_{3}-\nu_{4}\right)
\end{aligned}
$$

and reproduces the semiclassical term $\mathrm{C} 1 \mathrm{~b}$ in Appendix C.

\section{APPENDIX E}

\section{CORRELATION OF THE LIEU ET AL. SAMPLES: A QUANTUM CALCULATION}

This appendix presents a quantum-mechanical calculation that shows that the samples $I_{\Delta, \Delta t}^{2}(k)$ introduced in Equations (114), (115) and assumed by Lieu et al. (2015) to be independent are in fact correlated; furthermore, these correlations are shown to lead to the standard photon bunching penalty. Let $I_{a}(t)$ be the output of detector $a$; here $a=1$ or 2 . The corresponding quantum operator is

$$
I_{a}(t)=\int d \nu_{1} d \nu_{1}^{\prime} b_{a}^{\dagger}\left(\nu_{1}\right) b_{a}\left(\nu_{1}^{\prime}\right) e^{-i 2 \pi\left(\nu_{1}-\nu_{1}^{\prime}\right) t}
$$


We define the integral over the time interval $[k \Delta t,(k+1) \Delta t]$ as

$$
I_{a, \Delta t}(k)=\int_{k \Delta t}^{(k+1) \Delta t} d t I_{a}(t)
$$

The Lieu et al. detection scheme involves summing the squares of the differences $I_{\Delta t}^{d}(k)=I_{1, \Delta t}(k)-I_{2, \Delta t}(k)$ of the two time-averaged and sampled outputs of a beamsplitter-fed detector pair (Figures 1 and 9):

$$
S=\sum_{k=0}^{N-1}\left[I_{\Delta t}^{d}(k)\right]^{2}=(\Delta t)^{2} P_{N, \mathrm{LKD}}
$$

where $P_{N, \mathrm{LKD}}$ is defined in Equation (114). The mean value of a single sample is given by

$$
\left\langle\left[I_{\Delta t}^{d}(k)\right]^{2}\right\rangle=G_{11}(k)-G_{12}(k)-G_{21}(k)+G_{22}(k),
$$

where

$$
\begin{aligned}
G_{a b}(k)= & \left\langle I_{a, \Delta t}(k) I_{b, \Delta t}(k)\right\rangle \\
= & \int_{k \Delta t}^{(k+1) \Delta t} d t_{1} d t_{2} \int d \nu_{1} d \nu_{1}^{\prime} d \nu_{2} d \nu_{2}^{\prime} e^{-i 2 \pi\left(\nu_{1}-\nu_{1}^{\prime}\right) t_{1}} \\
& \times e^{-i 2 \pi\left(\nu_{2}-\nu_{2}^{\prime}\right) t_{2}}\left\langle b_{a}^{\dagger}\left(\nu_{1}\right) b_{a}\left(\nu_{1}^{\prime}\right) b_{b}^{\dagger}\left(\nu_{2}\right) b_{b}\left(\nu_{2}^{\prime}\right)\right\rangle \\
= & \int_{k \Delta t}^{(k+1) \Delta t} d t_{1} d t_{2} \int d \nu_{1} d \nu_{1}^{\prime} d \nu_{2} d \nu_{2}^{\prime} \\
& \times e^{-i 2 \pi\left(\nu_{1}-\nu_{1}^{\prime}\right) t_{1}} e^{-i 2 \pi\left(\nu_{2}-\nu_{2}^{\prime}\right) t_{2}} \\
& \times\left\{B_{a a}\left(\nu_{1}\right) \delta\left(\nu_{1}-\nu_{1}^{\prime}\right) B_{b b}\left(\nu_{2}\right) \delta\left(\nu_{2}-\nu_{2}^{\prime}\right)\right. \\
& +B_{a b}\left(\nu_{1}\right) \delta\left(\nu_{1}-\nu_{2}^{\prime}\right)\left[\delta_{a b}\right. \\
& \left.\left.+B_{b a}\left(\nu_{2}\right)\right] \delta\left(\nu_{2}-\nu_{1}^{\prime}\right)\right\} \\
\approx & \bar{\Gamma}_{a} \bar{\Gamma}_{b}(\Delta t)^{2}+\delta_{a b} \bar{\Gamma}_{a} \Delta t+\left|\int d \nu B_{a b}(\nu)\right|^{2}(\Delta t)^{2} ;
\end{aligned}
$$

we have made use of $\Delta \nu \Delta t \ll 1$ in approximating the third term. Note that the second term dominates in the short sample time regime, $\bar{\Gamma} \Delta t \ll 1$. Here $\bar{\Gamma}_{a}=\int d \nu B_{a a}(\nu)$ is the photon rate for detector $a$; the quantity $B_{a b}(\nu)$ is introduced in Section 3 through Equations (27), (36), and (37). Therefore,

$$
\left\langle\left[I_{\Delta t}^{d}(k)\right]^{2}\right\rangle \approx\left(\bar{\Gamma}_{1}+\bar{\Gamma}_{2}\right) \Delta t=\bar{\Gamma} \Delta t
$$

This is exactly what we expect given the discussion in Section 10: for small $\Delta t$, the value of $\left[I_{\Delta t}^{d}(k)\right]^{2}$ is unity if either detector receives a photon, and zero otherwise, and the probability of either receiving a photon is $\bar{\Gamma} \Delta t$. Thus, we conclude that mean value of the sum is

$$
\langle S\rangle=N \bar{\Gamma} \Delta t=\bar{\Gamma} T
$$

where $T=N \Delta t$ is the total time duration of the measurement.
To calculate the fluctuations of the sum $S$, we first define the quantity

$$
\begin{aligned}
F_{a b c d}(k)= & \left\langle I_{a, \Delta t}(k) I_{b, \Delta t}(k) I_{c, \Delta t}(0) I_{d, \Delta t}(0)\right\rangle \\
= & \int_{k \Delta t}^{(k+1) \Delta t} d t_{1} \int_{k \Delta t}^{(k+1) \Delta t} d t_{2} \int_{0}^{\Delta t} d t_{3} \\
& \times \int_{0}^{\Delta t} d t_{4}\left\langle I_{a}\left(t_{1}\right) I_{b}\left(t_{2}\right) I_{c}\left(t_{3}\right) I_{d}\left(t_{4}\right)\right\rangle .
\end{aligned}
$$

We wish to evaluate the correlation

$$
C_{\Delta t}(k)=\left\langle\left[I_{\Delta t}^{d}(k)\right]^{2}\left[I_{\Delta t}^{d}(0)\right]^{2}\right\rangle .
$$

We may easily express $C_{\Delta t}(k)$ in terms of $F_{a b c d}(k)$ :

$$
\begin{aligned}
C_{\Delta t}(k)= & F_{1111}(k)-F_{2111}(k)-F_{1211}(k)+F_{2211}(k) \\
& -F_{1121}(k)+F_{2121}(k)+F_{1221}(k)-F_{2221}(k) \\
& -F_{1112}(k)+F_{2112}(k)+F_{1212}(k)-F_{2212}(k) \\
& +F_{1122}(k)-F_{2122}(k)-F_{1222}(k)+F_{2222}(k) .
\end{aligned}
$$

As usual, evaluation of $F_{a b c d}(k)$ involves an eighth-order moment of photon operators,

$$
\begin{aligned}
F_{a b c d}(k)= & \int_{k \Delta t}^{(k+1) \Delta t} d t_{1} \int_{k \Delta t}^{(k+1) \Delta t} d t_{2} \int_{0}^{\Delta t} d t_{3} \int_{0}^{\Delta t} d t_{4} \\
& \times \int_{0}^{\infty} d \nu_{1} d \nu_{1}^{\prime} d \nu_{2} d \nu_{2}^{\prime} d \nu_{3} d \nu_{3}^{\prime} d \nu_{4} d \nu_{4}^{\prime} \\
& \times e^{-i 2 \pi\left(\nu_{1}-\nu_{1}^{\prime}\right) t_{1}} e^{-i 2 \pi\left(\nu_{2}-\nu_{2}^{\prime}\right) t_{2}} e^{-i 2 \pi\left(\nu_{3}-\nu_{3}^{\prime}\right) t_{3}} \\
& \times e^{-i 2 \pi\left(\nu_{4}-\nu_{4}^{\prime}\right) t_{4}}\left\langle b_{a}^{\dagger}\left(\nu_{1}\right) b_{a}\left(\nu_{1}^{\prime}\right) b_{b}^{\dagger}\left(\nu_{2}\right)\right. \\
& \left.\times b_{b}\left(\nu_{2}^{\prime}\right) b_{c}^{\dagger}\left(\nu_{3}\right) b_{c}\left(\nu_{3}^{\prime}\right) b_{d}^{\dagger}\left(\nu_{4}\right) b_{d}\left(\nu_{4}^{\prime}\right)\right\rangle .
\end{aligned}
$$

Pairwise combination of the operators gives $4 !=24$ terms. It is not difficult to show that the (12)(34) permutation gives

$$
F_{a b c d}^{(12)(34)}(k)=G_{a b}(k) G_{c d}(0),
$$

and inserting this result into Equation (E9) gives

$$
\begin{aligned}
C_{\Delta t}^{(12)(34)}(k) & =\left[G_{11}(0)-G_{12}(0)-G_{21}(0)+G_{22}(0)\right]^{2} \\
& =\left\langle\left[I_{\Delta t}^{d}(k)\right]^{2}\right\rangle^{2} .
\end{aligned}
$$

This term will therefore contribute $\langle S\rangle^{2}$ when calculating $\left\langle S^{2}\right\rangle$, which will subtract out when we calculate the variance of $S$. As in Appendix D, the (12)(34) permutation contributes to the mean value rather than to the fluctuations.

As before, the operator pairing corresponding to the (1432) permutation is responsible for the Poisson and bunching noise:

$$
\begin{aligned}
F_{a b c d}^{(1432)}(k)= & \int_{k \Delta t}^{(k+1) \Delta t} d t_{1} \int_{k \Delta t}^{(k+1) \Delta t} d t_{2} \int_{0}^{\Delta t} d t_{3} \int_{0}^{\Delta t} d t_{4} \\
& \times \int_{0}^{\infty} d \nu_{1} d \nu_{1}^{\prime} d \nu_{2} d \nu_{2}^{\prime} d \nu_{3} d \nu_{3}^{\prime} d \nu_{4} d \nu_{4}^{\prime} \\
& \times e^{-i 2 \pi\left(\nu_{1}-\nu_{1}^{\prime}\right) t_{1}} e^{-i 2 \pi\left(\nu_{2}-\nu_{2}^{\prime}\right) t_{2}} e^{-i 2 \pi\left(\nu_{3}-\nu_{3}^{\prime}\right) t_{3}}
\end{aligned}
$$




$$
\begin{aligned}
& \times e^{-i 2 \pi\left(\nu_{4}-\nu_{4}^{\prime}\right) t_{4}}\left\langle b_{a}^{\dagger}\left(\nu_{1}\right) b_{d}\left(\nu_{4}^{\prime}\right)\right\rangle \\
& \times\left\langle b_{a}\left(\nu_{1}^{\prime}\right) b_{b}^{\dagger}\left(\nu_{2}\right)\right\rangle\left\langle b_{b}\left(\nu_{2}^{\prime}\right) b_{c}^{\dagger}\left(\nu_{3}\right)\right\rangle \\
& \times\left\langle b_{c}\left(\nu_{3}^{\prime}\right) b_{d}^{\dagger}\left(\nu_{4}\right)\right\rangle \\
= & \int_{k \Delta t}^{(k+1) \Delta t} d t_{1} \int_{k \Delta t}^{(k+1) \Delta t} d t_{2} \int_{0}^{\Delta t} d t_{3} \\
& \times \int_{0}^{\Delta t} d t_{4} \int_{0}^{\infty} d \nu_{1} d \nu_{2} d \nu_{3} d \nu_{4} \\
& \times e^{-i 2 \pi\left(\nu_{1}-\nu_{2}\right) t_{1}} e^{-i 2 \pi\left(\nu_{2}-\nu_{3}\right) t_{2}} e^{-i 2 \pi\left(\nu_{3}-\nu_{4}\right) t_{3}} \\
& \times e^{-i 2 \pi\left(\nu_{4}-\nu_{1}\right) t_{4}} B_{a d}\left(\nu_{1}\right) \\
& \times\left[\delta_{a b}+B_{b a}\left(\nu_{2}\right)\right]\left[\delta_{b c}+B_{c b}\left(\nu_{3}\right)\right] \\
& \times\left[\delta_{c d}+B_{d c}\left(\nu_{4}\right)\right] .
\end{aligned}
$$

This expression leads to eight terms; among these is the term that gives rise to the $\mathrm{C} 1 \mathrm{~b}$ contribution in the semiclassical and quantum calculations in Appendices B and D:

$$
\begin{aligned}
F_{a b c d}^{(1432), \mathrm{C} 1 \mathrm{~b}}(k)= & \int_{k \Delta t}^{(k+1) \Delta t} d t_{1} \int_{k \Delta t}^{(k+1) \Delta t} d t_{2} \\
& \times \int_{0}^{\Delta t} d t_{3} \int_{0}^{\Delta t} d t_{4} \int_{0}^{\infty} d \nu_{1} d \nu_{2} d \nu_{3} d \nu_{4} \\
& \times e^{-i 2 \pi \nu_{1}\left(t_{1}-t_{4}\right)} e^{-i 2 \pi \nu_{2}\left(t_{2}-t_{1}\right)} \\
& \times e^{-i 2 \pi \nu_{3}\left(t_{3}-t_{2}\right)} e^{-i 2 \pi \nu_{4}\left(t_{4}-t_{3}\right)} \\
& \times \delta_{a b} \delta_{c d} B_{a c}\left(\nu_{1}\right) B_{c a}\left(\nu_{3}\right)
\end{aligned}
$$

The $\nu_{2}$ integral gives $\delta\left(t_{2}-t_{1}\right)$ while the $\nu_{4}$ integral gives $\delta\left(t_{4}-t_{3}\right) ;$ therefore

$$
\begin{aligned}
F_{a b c d}^{(1432), \mathrm{C} 1 \mathrm{~b}}(k)= & \int_{k \Delta t}^{(k+1) \Delta t} d t_{1} \int_{0}^{\Delta t} d t_{3} \int_{0}^{\infty} d \nu_{1} d \nu_{3} \\
& \times e^{-i 2 \pi \nu_{1}\left(t_{1}-t_{3}\right)} e^{-i 2 \pi \nu_{3}\left(t_{3}-t_{1}\right)} \\
& \times \delta_{a b} \delta_{c d} B_{a c}\left(\nu_{1}\right) B_{c a}\left(\nu_{3}\right) .
\end{aligned}
$$

Note that

$$
B_{a c}\left(\nu_{1}\right) B_{c a}\left(\nu_{3}\right)=\frac{1}{4} n\left(\nu_{1}\right) n\left(\nu_{3}\right)
$$

regardless of the choice of indices. Furthermore,

$$
\begin{gathered}
\int_{0}^{\infty} d \nu_{1} d \nu_{3} n\left(\nu_{1}\right) n\left(\nu_{3}\right) e^{-i 2 \pi \nu_{1} \tau} e^{+i 2 \pi \nu_{3} \tau} \\
=\int_{0}^{\infty} d \nu d \nu^{\prime} n(\nu) n\left(\nu+\nu^{\prime}\right) e^{i 2 \pi \nu^{\prime} \tau} \\
=\int_{0}^{\infty} d \nu^{\prime} S_{\Gamma}\left(\nu^{\prime}\right) e^{i 2 \pi \nu^{\prime} \tau}=A_{\Gamma}(\tau),
\end{gathered}
$$

where $A_{\Gamma}(\tau)$ is the Fourier transform of $S_{\Gamma}(\nu)$ and represents the time autocorrelation function of the photon rate fluctuations. Note that $A_{\Gamma}(\tau)$ decays on a timescale $\tau \sim 1 / \Delta \nu$, and that $A(0)=\bar{\Gamma}^{2}$. Thus

$$
\begin{aligned}
F_{a b c d}^{(1432), \mathrm{C} 1 \mathrm{~b}}(k)= & \frac{1}{4} \delta_{a b} \delta_{c d} \int_{k \Delta t}^{(k+1) \Delta t} d t_{1} \\
& \times \int_{0}^{\Delta t} d t_{3} A_{\Gamma}\left(t_{1}-t_{3}\right) \\
\approx & \frac{1}{4} \delta_{a b} \delta_{c d}(\Delta t)^{2} A_{\Gamma}(k \Delta t)
\end{aligned}
$$

where the approximation holds because $\Delta t \Delta \nu \ll 1$. The (1432) pairing also contributes a term that corresponds to Poisson noise, labeled D1 in the semiclassical calculation:

$$
F_{a b c d}^{(1432), \mathrm{D} 1}(k)=\frac{1}{2} \delta_{a b} \delta_{c d} \delta_{a c} \delta_{k, 0} \Delta t \bar{\Gamma}
$$

Of the sixteen terms in Equation (E9), the only nonzero contributions for the $\mathrm{C} 1 \mathrm{~b}$ piece of the (1432) permutation come from $F_{1111}, F_{1122}, F_{2211}$, and $F_{2222}$, due to the $\delta_{a b} \delta_{c d}$ factor; and all have the same sign. For the D1 piece, the additional $\delta_{a c}$ factor means that only $F_{1111}$ and $F_{2222}$ contribute. These two pieces give a contribution to $C_{\Delta t}(k)$ given by

$$
C_{\Delta t}^{(1432), \mathrm{C} 1 \mathrm{~b}+\mathrm{D} 1}(k) \approx \bar{\Gamma} \Delta t \delta_{k, 0}+(\Delta t)^{2} A_{\Gamma}(k \Delta t) .
$$

The second term in this expression shows that the quantities $\left[I_{\Delta t}^{d}(k)\right]^{2}$ are indeed correlated, in contradiction to the assumption of Lieu et al. (2015). The corresponding contribution to the variance of $S$ (Equation (E3)) is

$$
\begin{aligned}
\sigma_{S}^{2} & =\left\langle S^{2}\right\rangle-\langle S\rangle^{2} \\
& =\left[\sum_{k, l=0}^{N-1} C_{\Delta t}(k-l)\right]-\langle S\rangle^{2} \\
& =\sum_{k, l=0}^{N-1}\left\{\bar{\Gamma} \Delta t \delta_{k l}+(\Delta t)^{2} A_{\Gamma}[(k-l) \Delta t]\right\}+\ldots \\
& \approx N \bar{\Gamma} \Delta t+N(\Delta t)^{2} \frac{n^{2} \Delta \nu}{\Delta t}+\ldots \\
& =\bar{\Gamma} T(1+n)+\ldots
\end{aligned}
$$

where I made use of

$$
\begin{aligned}
\sum_{l} A_{\Gamma}[(k-l) \Delta t] & \approx \frac{1}{\Delta t} \int_{-\infty}^{+\infty} A_{\Gamma}(\tau) d \tau \\
& =\frac{1}{\Delta t} \int_{0}^{\infty} n^{2}(\nu) d \nu=\frac{n^{2} \Delta \nu}{\Delta t}
\end{aligned}
$$

$T=N \Delta t$, and $\bar{\Gamma}=n \Delta \nu$. Using Equation (E7), we find

$$
\frac{\sigma_{N, \mathrm{LKD}}^{2}}{\left\langle P_{N, \mathrm{LKD}}\right\rangle}=\frac{\sigma_{S}^{2}}{\langle S\rangle^{2}} \approx \frac{1+n}{\bar{\Gamma} T}+\ldots
$$

This expression agrees with the other results presented in this paper (Equation (136)) but contradicts the fundamental result of Lieu et al. (2015) (Equation (135)).

\section{APPENDIX F \\ EQUIVALENCE OF QUANTUM AND SEMICLASSICAL APPROACHES}

In this appendix, I use a straightforward extension of the arguments developed by Sudarshan (1963) to show that the full quantum-mechanical calculation of photon bunching is equivalent to a semiclassical calculation that makes use of a compound Poisson random process with a stochastically varying count rate. The equivalence is shown by comparing the generating functionals, defined as

$$
G[s]=\left\langle\exp \left[\int_{-\infty}^{+\infty} d t s(t) I(t)\right]\right\rangle .
$$

The semiclassical and quantum-mechanical versions will be denoted by $G^{(\mathrm{sc})}[s]$ and $G^{(\mathrm{qm})}[s]$, respectively. These generating 
functions fully encode the statistics of the photocurrent $I(t)$; the statistics must be the same if $G^{(\mathrm{sc})}[s]=G^{(\mathrm{qm})}[s]$.

A Poisson process with a deterministic time-varying rate $\Gamma(t)$ obeys Equation (A6):

$$
\begin{aligned}
\langle\exp & {\left.\left[s \int_{t_{k}}^{t_{k+1}} I(t) d t\right]\right\rangle_{y} } \\
& =\exp \left\{\left[\int_{t_{k}}^{t_{k+1}} \Gamma(t) d t\right]\left(e^{s}-1\right)\right\} .
\end{aligned}
$$

If we make the time interval small enough, we may approximate

$$
\left\langle\exp \left[s \int_{t_{k}}^{t_{k+1}} I(t) d t\right]\right\rangle_{y} \approx \exp \left\{\Gamma\left(t_{k}\right) \Delta t_{k}\left(e^{s}-1\right)\right\} .
$$

If the intervals $\left[t_{k}, t_{k+1}\right]$ span the region over which $s(t)$ is nonzero, we may write

$$
\int_{-\infty}^{+\infty} d t s(t) I(t) \approx \sum_{k} s\left(t_{k}\right) \int_{t_{k}}^{t_{k+1}} I(t) d t .
$$

Making use of the independence of the subinterval counts $\{y\}$, we have

$$
\begin{aligned}
G[s] & \approx\left\langle\exp \left[\sum_{k} s\left(t_{k}\right) \int_{t_{k}}^{t_{k+1}} I(t) d t\right]\right\rangle_{y} \\
& =\prod_{k}\left\langle\exp \left[s\left(t_{k}\right) \int_{t_{k}}^{t_{k+1}} I(t) d t\right]\right\rangle_{y} \\
& \approx \prod_{k} \exp \left\{\Gamma\left(t_{k}\right) \Delta t_{k}\left(e^{s\left(t_{k}\right)}-1\right)\right\} \\
& =\exp \left\{\sum_{k} \Gamma\left(t_{k}\right) \Delta t_{k}\left(e^{s\left(t_{k}\right)}-1\right)\right\}
\end{aligned}
$$

and by taking the continuum limit we find

$$
G[s]=\exp \left\{\int_{-\infty}^{+\infty} d t \Gamma(t)\left(e^{s(t)}-1\right)\right\} .
$$

If we now allow the rate $\Gamma(t)$ to be stochastic instead of deterministic, we must also perform an average over $\Gamma(t)$. We obtain a formal expression for the semiclassical generating function by writing this average as a functional integral

$$
\begin{aligned}
G^{(\mathrm{sc})}[s]= & \int[d \Gamma(t)] f[\Gamma(t)] \\
& \times \exp \left\{\int_{-\infty}^{+\infty} d t \Gamma(t)\left(e^{s(t)}-1\right)\right\},
\end{aligned}
$$

where $f[\Gamma(t)]$ represents the probability density functional for the rate process $\Gamma(t)$ (Ueda 1989) and $[d \Gamma(t)]$ is the functional integration measure.

We now show that the quantum generating functional may also be written in this manner and obtain an expression for the resulting probability density $f[\Gamma(t)]$. The quantum-mechanical averages require traces over the thermal density matrix given by Equation (3):

$$
\begin{aligned}
G^{(\mathrm{qm})}[s]= & \left\langle\exp \left[\int_{-\infty}^{+\infty} d t s(t) I(t)\right]\right\rangle \\
= & \operatorname{Tr}\left\{\exp \left[\int_{-\infty}^{+\infty} d t s(t) b^{\dagger}(t) b(t)\right] \rho\right\} . \\
= & \operatorname{Tr}\left\{\operatorname { e x p } \left[\int_{0}^{\infty} d \nu_{1} d \nu_{2} \hat{s}\left(\nu_{1}-\nu_{2}\right)\right.\right. \\
& \left.\left.\times b^{\dagger}\left(\nu_{1}\right) b\left(\nu_{2}\right)\right] \rho\right\} .
\end{aligned}
$$

where $\hat{s}(\nu)$ is the Fourier transform of $s(t)$,

$$
\hat{s}(\nu)=\int_{-\infty}^{+\infty} d t s(t) e^{i 2 \pi \nu t} .
$$

In the following discussion, we will find it useful to switch between operators labeled by a continuous frequency index and a discrete approximation using

$$
\int_{0}^{\infty} d \nu_{1} d \nu_{2} \hat{s}\left(\nu_{1}-\nu_{2}\right) b^{\dagger}\left(\nu_{1}\right) b\left(\nu_{2}\right) \leftrightarrow \sum_{i j} S_{i j} b_{i}^{\dagger} b_{j}
$$

where

$$
b_{i}=\frac{1}{\sqrt{\Delta \nu_{i}}} \int_{\nu_{i}}^{\nu_{i}+\Delta \nu_{i}} b(\nu)
$$

and similarly for $b_{i}^{\dagger}$, and therefore $\left[b_{i}, b_{j}^{\dagger}\right]=\delta_{i j}$, while

$$
S_{i j}=\hat{s}\left(\nu_{i}-\nu_{j}\right) \sqrt{\Delta \nu_{i} \Delta \nu_{j}}
$$

is a Hermitian matrix by virtue of $\hat{s}(-\nu)=\hat{s}^{*}(\nu)$.

The coherent state representation is convenient for calculating $G^{(\mathrm{qm})}[s]$. The coherent states (Glauber 1963) are given by

$$
|z\rangle=\exp \left(\sum_{i} z_{i} b_{i}^{\dagger}\right)|0\rangle
$$

and satisfy the normalization

$$
\left\langle z \mid z^{\prime}\right\rangle=\exp \left(\sum_{i} z_{i}^{*} z_{i}\right)=e^{z^{\dagger} z},
$$

where $z$ represents the column vector with components $\left\{z_{i}\right\}$ and $z^{\dagger}$ is its Hermitian conjugate, a row vector with components $\left\{z_{i}^{*}\right\}$. The coherent states satisfy the overcompleteness relation

$$
1=\int d \mu(z) e^{-z^{\dagger} z}|z\rangle\langle z|
$$

where the integration measure is

$$
d \mu(z)=\prod_{i} \frac{d\left(\operatorname{Re} z_{i}\right) d\left(\operatorname{Im} z_{i}\right)}{\pi} .
$$

The thermal density matrix has a diagonal coherent-state representation

$$
\rho=\operatorname{det}\left(N^{-1}\right) \int d \mu(z) e^{-z^{\dagger} z} e^{-z^{\dagger} N^{-1} z}|z\rangle\langle z|
$$

where $N$ is a diagonal matrix of mode occupation numbers with elements

$$
N_{i j}=n_{i} \delta_{i j}=\frac{1}{e^{x_{i}}-1} \delta_{i j} .
$$

Thermal averages may be computed using this representation,

$$
\operatorname{Tr}(A \rho)=\operatorname{det}\left(N^{-1}\right) \int d \mu(z) e^{-z^{\dagger} z} e^{-z^{\dagger} N^{-1} z}\langle z|A| z\rangle .
$$


The operator we are interested in has the form

$$
A=\exp \left(\sum_{i j} S_{i j} b_{i}^{\dagger} b_{j}\right)=\exp \left(b^{\dagger} S b\right)
$$

where we use the vector notation for the photon operators in which $b$ represents a column vector with elements $\left\{b_{i}\right\}$ and $b^{\dagger}$ represents its Hermitian conjugate. Coherent-state matrix elements may be evaluated using the normal ordering theorem (Blasiak et al. 2007)

$$
\exp \left[b^{\dagger} S b\right]=: \exp \left[b^{\dagger}\left(e^{S}-1\right) b\right]:
$$

which gives a compact result for the quantum-mechanical generating functional,

$$
\begin{aligned}
G^{(\mathrm{qm})}[s]= & \operatorname{det}\left(N^{-1}\right) \int d \mu(z) e^{-z^{\dagger} z} e^{-z^{\dagger} N^{-1} z} \\
& \times\left\langle z\left|: \exp \left[b^{\dagger}\left(e^{S}-1\right) b\right]:\right| z\right\rangle \\
= & \operatorname{det}\left(N^{-1}\right) \int d \mu(z) e^{-z^{\dagger} z} e^{-z^{\dagger} N^{-1} z} \\
& \times \exp \left[z^{\dagger}\left(e^{S}-1\right) z\right]\langle z \mid z\rangle \\
= & \operatorname{det}\left(N^{-1}\right) \int d \mu(z) \exp \left[-z^{\dagger}\left(N^{-1}-e^{S}+1\right) z\right] \\
= & \frac{\operatorname{det}\left(N^{-1}\right)}{\operatorname{det}\left(N^{-1}-e^{S}+1\right)} \\
= & \frac{1}{\operatorname{det}\left[1-N\left(e^{S}-1\right)\right]} \\
= & \exp \left\{-\operatorname{Tr} \ln \left[1-N\left(e^{S}-1\right)\right]\right\} .
\end{aligned}
$$

Here we have made use of the complex Gaussian integral (Negele \& Orland 1988)

$$
\int d \mu(z) \exp \left[-z^{\dagger} B z-c^{\dagger} z-z^{\dagger} d\right]=\frac{\exp \left[c^{\dagger} B^{-1} d\right]}{\operatorname{det} B} .
$$

Equation (F20) resembles other results for thermal radiation, e.g., those of Beenakker (1998).

Although Equation (F20) is a relatively simple expression for the quantum-mechanical generating function, it is not easy to compare this result to our semiclassical generating functional given by Equation (F6). If we hold off the $z$-integration, we have

$$
G^{(\mathrm{qm})}[s]=\operatorname{det}\left(N^{-1}\right) \int d \mu(z) \exp \left[-z^{\dagger} N^{-1} z-z^{\dagger}\left(e^{S}-1\right) z\right] .
$$

In the continuum limit, the second term in the argument of the exponential is

$$
z^{\dagger}\left(e^{S}-1\right) z=\int d \nu d \nu^{\prime} z^{*}(\nu)\left(e^{S}-1\right)_{\nu, \nu^{\prime}} z\left(\nu^{\prime}\right) .
$$

Now

$$
\begin{aligned}
\left(e^{S}-1\right)_{\nu, \nu^{\prime}}= & \hat{s}\left(\nu-\nu^{\prime}\right)+\frac{1}{2 !} \int d \nu_{1} \hat{s}\left(\nu-\nu_{1}\right) \\
& \times \hat{s}\left(\nu_{1}-\nu^{\prime}\right)+\ldots
\end{aligned}
$$

$$
\begin{aligned}
= & \int d t_{1} s\left(t_{1}\right)\left[e^{i 2 \pi\left(\nu-\nu^{\prime}\right) t_{1}}\right] \\
& +\frac{1}{2 !} \int d t_{1} d t_{2} s\left(t_{1}\right) s\left(t_{2}\right) \\
& \times \int d \nu_{1} e^{i 2 \pi\left(\nu-\nu_{1}\right) t_{1}} e^{i 2 \pi\left(\nu_{1}-\nu^{\prime}\right) t_{2}}+\ldots \\
= & \int d t_{1}\left[s\left(t_{1}\right)+\frac{1}{2 !} s^{2}\left(t_{1}\right)+\ldots\right] e^{i 2 \pi\left(\nu-\nu^{\prime}\right) t_{1}} \\
= & \int d t\left[e^{s(t)}-1\right] e^{i 2 \pi\left(\nu-\nu^{\prime}\right) t} .
\end{aligned}
$$

Thus we obtain

$$
z^{\dagger}\left(e^{S}-1\right) z=\int d t \Gamma(t \mid z)\left(e^{s(t)}-1\right)
$$

where

$$
\Gamma(t \mid z)=\int d \nu d \nu^{\prime} e^{i 2 \pi\left(\nu-\nu^{\prime}\right) t} z^{*}(\nu) z\left(\nu^{\prime}\right)
$$

We thus conclude that the quantum-mechanical generating functional may be written in a form identical to that of a compound Poisson process as expressed in Equation (F6),

$$
\begin{aligned}
G^{(\mathrm{qm})}[s]= & \exp [-\operatorname{Tr} \ln N] \int d \mu(z) \exp \left(-z^{\dagger} N^{-1} z\right) \\
& \times \exp \left[\int d t \Gamma(t \mid z)\left(e^{s(t)}-1\right)\right] \\
= & \int[d \Gamma(t)] f[\Gamma(t) \mid N] \\
& \times \exp \left\{\int_{-\infty}^{+\infty} d t \Gamma(t)\left(e^{s(t)}-1\right)\right\} \\
= & G^{(\mathrm{sc})}[s]
\end{aligned}
$$

provided that the probability density functional for the stochastic rate process is given by

$$
\begin{aligned}
f[\Gamma(t) \mid N]= & \exp [-\operatorname{Tr} \ln N] \int d \mu(z) \\
& \times \exp \left(-z^{\dagger} N^{-1} z\right) \delta[\Gamma(t)-\Gamma(t \mid z)]
\end{aligned}
$$

and where $\Gamma(t \mid z)$ is given by Equation (F24). Note that while $z(\nu)$ has a Gaussian distribution with variance $n(\nu), \Gamma(t \mid z)$ is a quadratic form of $z(\nu)$ and therefore is not strictly Gaussian.

It is not difficult to demonstrate that

$$
\begin{aligned}
& \exp [-\operatorname{Tr} \ln N] \int d \mu(z) \\
& \quad \times \exp \left(-z^{\dagger} N^{-1} z\right) z\left(\nu_{1}\right) z^{*}\left(\nu_{2}\right)=n\left(\nu_{1}\right) \delta\left(\nu_{1}-\nu_{2}\right)
\end{aligned}
$$

while

$$
\begin{aligned}
\exp [ & -\operatorname{Tr} \ln N] \int d \mu(z) \\
& \times \exp \left(-z^{\dagger} N^{-1} z\right) z\left(\nu_{1}\right) z^{*}\left(\nu_{2}\right) z\left(\nu_{2}\right) z^{*}\left(\nu_{4}\right) \\
= & n\left(\nu_{1}\right) n\left(\nu_{3}\right) \delta\left(\nu_{1}-\nu_{2}\right) \delta\left(\nu_{3}-\nu_{4}\right) \\
& +n\left(\nu_{1}\right) n\left(\nu_{3}\right) \delta\left(\nu_{1}-\nu_{4}\right) \delta\left(\nu_{3}-\nu_{2}\right) .
\end{aligned}
$$


Thus, the mean of the equivalent stochastic rate process is

$$
\begin{aligned}
\left\langle\Gamma\left(t_{1}\right)\right\rangle & =\int d \mu[\Gamma(t)] f[\Gamma(t) \mid n] \Gamma\left(t_{1}\right) \\
& =\exp [-\operatorname{Tr} \ln N] \int d \mu(z) \exp \left(-z^{\dagger} N^{-1} z\right) \Gamma\left(t_{1} \mid z\right) \\
& =\int d \nu d \nu^{\prime} e^{i 2 \pi\left(\nu-\nu^{\prime}\right) t_{1}} n(\nu) \delta\left(\nu-\nu^{\prime}\right) \\
& =\int d \nu n(\nu)=\bar{\Gamma}
\end{aligned}
$$

which is the expected result. Meanwhile, the second moment is

$$
\begin{aligned}
\left\langle\Gamma\left(t_{1}\right) \Gamma\left(t_{2}\right)\right\rangle= & \int d \nu_{1} d \nu_{2} d \nu_{3} d \nu_{4} e^{i 2 \pi\left(\nu_{1}-\nu_{2}\right) t_{1}} e^{i 2 \pi\left(\nu_{3}-\nu_{4}\right) t_{2}} \\
& \times\left[n\left(\nu_{1}\right) \delta\left(\nu_{1}-\nu_{2}\right) n\left(\nu_{3}\right) \delta\left(\nu_{3}-\nu_{4}\right)\right. \\
& \left.+n\left(\nu_{1}\right) \delta\left(\nu_{1}-\nu_{4}\right) n\left(\nu_{3}\right) \delta\left(\nu_{3}-\nu_{2}\right)\right] \\
= & \int d \nu_{1} d \nu_{3}\left[n\left(\nu_{1}\right) n\left(\nu_{3}\right)\right. \\
& \left.+e^{i 2 \pi\left(\nu_{1}-\nu_{3}\right)\left(t_{1}-t_{2}\right)} n\left(\nu_{1}\right) n\left(\nu_{3}\right)\right] \\
= & \bar{\Gamma}^{2}+\int d \nu S_{\Gamma}(\nu) e^{i 2 \pi \nu\left(t_{1}-t_{2}\right)}
\end{aligned}
$$

These results coincide with Equations (57) and (58).

\section{REFERENCES}

Beenakker, C. W. J. 1998, PhRvL, 81, 1829

Blasiak, P., Horzela, A., Penson, K. A., Solomon, A. I., \& Duchamp, G. H. E. 2007, AmJPh, 75, 639

Dicke, R. H. 1946, RScI, 17, 268

Gehrels, N. 1986, ApJ, 303, 336

Glauber, R. J. 1963, PhRvL, 10, 84

Hanbury Brown, R., \& Twiss, R. Q. 1956, Natur, 177, 27

Hanbury Brown, R., \& Twiss, R. Q. 1957, RSPSA, 242, 300

Harwit, M. 1960, PhRv, 120, 1551

Held, D. N., \& Kerr, A. R. 1978, ITMTT, 26, 49

Kelley, P. L., \& Kleiner, W. H. 1964, PhRv, 136, A316

Lieu, R., Kibble, T. W. B., \& Duan, L. 2015, ApJ, 798, 67

Mandel, L. 1958, PPS, 72, 1037

Mandel, L. 1959, PPS, 74, 233

Morgan, B. L., \& Mandel, L. 1966, PhRvL, 16, 1012

Negele, J. W., \& Orland, H. 1988, Quantum Many-particle Systems (Boulder, CO: Westview), 34

Nityananda, R. 1994, in Proc. 158th IAU Symp., Very High Angular Resolution Imaging, ed. J. G. Robertson \& W. J. Tango (Dordrecht: Springer), 11

Picinbono, B., Benjaballah, C., \& Pouget, J. 1970, JMP, 11, 2166

Pozar, D. M. 2012, Microwave Engineering (4th ed.; New York: Wiley)

Quinlan, F., Fortier, T. M., Jiang, H., \& Diddams, S. A. 2013, JOSAB, 30,1775

Rousseau, M. 1971, JOSA, 61, 1307

Schoelkopf, R. J., Burke, P. J., Kozhevnikov, A. A., Prober, D. E., \& Rooks, M. J. 1997, PhRvL, 78, 3370

Schoelkopf, R. J., Moseley, S. H., Stahle, C. M., Wahlgren, P., \& Delsing, P. 1999, ITAS, 9, 2935

Schottky, W. 1918, AnP, 57, 541

Sudarshan, E. C. G. 1963, PhRvL, 10, 277

Tucker, J. R., \& Millea, M. F. 1978, ApPhL, 33, 611

Ueda, M. 1989, PhRvA, 40, 1097

Zmuidzinas, J. 2003, ApOpt, 42, 4989 